\title{
Ethnobotany, Phytochemistry and Pharmacological Effects of Plants in Genus Cynanchum Linn. (Asclepiadaceae)
}

\author{
Lu Han ${ }^{1,2, \dagger}$, Xiuping Zhou ${ }^{1,2, \dagger}$, Mengmeng Yang ${ }^{1,2}$, Li Zhou ${ }^{1,2}$, Xinxin Deng ${ }^{1,2}$, Shijie Wei ${ }^{1,2}$, \\ Wenping Wang ${ }^{1,2}$, Zhizhong Wang ${ }^{1,2}$, Xue Qiao ${ }^{1,2}$ and Changcai Bai ${ }^{1,2, * \text { (D) }}$ \\ 1 Key Laboratory of Hui Ethnic Medicine Modernization, Ministry of Education, Ningxia Medical University, \\ Yinchuan 750004, China; lulu2008han@163.com (L.H.); 18295498705@163.com (X.Z.); \\ 15809605110@163.com (M.Y.); zhouli1028@163.com (L.Z.); dengxx1012@163.com (X.D.); \\ weishijie818@sohu.com (S.W.); wpwang2015@163.com (W.W.); pxwzz@163.com (Z.W.); \\ snow_36man@126.com (X.Q.) \\ 2 Ningxia Research Center of Modern Hui Medicine Engineering and Technology; Yinchuan 750004, China \\ * Correspondence: changcaibai@163.com; Tel./Fax: +86-951-6880-582 \\ + These authors contribute to the paper equally.
}

Received: 28 April 2018; Accepted: 14 May 2018; Published: 16 May 2018

\begin{abstract}
Genus Cynanchum L. belongs to the family Asclepiadaceae, which comprise more than 200 species distributed worldwide. In Chinese medical practice, numerous drugs (such as tablets and powders) containing different parts of plants of this genus are used to treat snake bites, bruises, osteoblasts, rheumatoid arthritis and tumors. A search for original articles published on the cynanchum genus was performed by using several resources, including Flora of China Official Website and various scientific databases, such as PubMed, SciFinder, the Web of Science, Science Direct, and China Knowledge Resource Integrated (CNKI). Advances in the botanical, ethnomedicinal, phytochemical, and pharmacological studies of this genus are reviewed in this paper. Results showed that more than 440 compounds, including C21 steroids, steroidal saponins, alkaloids, flavonoids and terpene, have been isolated and identified from Cynanchum plants up to now. In vivo and in vitro studies have shown that plants possess an array of biological activities, including anti-tumor, neuroprotective and anti-fungal effects. Popular traditional prescription of Cynanchum sp. was also summed up in this paper. However, many Cynanchum species have received little or no attention. Moreover, few reports on the clinical use and toxic effects of Cynanchum sp. are available. Further attention should be focused on the study of these species to gather information on their respective toxicology data and relevant quality-control measures and clinical value of the crude extracts, active compounds, and bioactive metabolites from this genus. Further research on Cynanchum sp. should be conducted, and bioactivity-guided isolation strategies should be emphasized. In addition, systematic studies of the chemical composition of plants should be enhanced.
\end{abstract}

Keywords: Cynanchum L.; ethnobotany; phytochemistry; pharmacological effects; review

\section{Introduction}

Cynanchum L. is a large genus in the Asclepiadaceae family comprising approximately 200 species. Many of these plants have been used for a long time in traditional Chinese medicine (TCM) for the treatment of common and chronic diseases. Plants of this genus are distributed worldwide, including in East Africa, the Mediterranean region, the tropical zone of Europe, and the subtropical and temperate zones of Asia [1]. A total of 53 species and 12 varieties are native to the southwestern region of China [2]. However, only 33 species of the genus Cynanchum have been systematically studied to date [3]. 
Cynanchum L. is an important taxonomic group in the Asclepiadaceae family because numerous species of this genus have several application prospects other than in the field of medicine. These species of Cynanchum include C. sibiricum, C. chinense, C. auriculatum, C. officinale, C. bungei, C. otophyllum, C. corymbosum, C. amplexicaule, C. forrestii, C. stauntonii, C. vincetoxicum, C. inamoenum, C. atratum (CA), C. glaucescens, C. paniculatum, C. komarovii, C. versicolor, C. chekiangense and C. mooreanum (http:/ / frps.eflora.cn/frps/Cynanchum). These plants are traditionally used to treat snake bites, bruises, osteoblasts, rheumatoid arthritis and tumors. Some plants are poisonous; thus, they are used to kill agricultural pests and tigers because of their higher toxicity than other plants [1]. In addition, modern pharmacological studies showed that Cynanchum plants exert significant immune regulation, anti-oxidation, anti-tumor and other pharmacological effects [4].

Given the high medicinal value of anti-tumor, immune regulation and anti-oxidation of Cynanchum, a growing number of studies have been carried out on the chemical composition of the genus [5]. At present, 450 compounds from Cynanchum sp. have been isolated. Results showed that C21 steroids are the main chemical constituents of this genus, as well as acetophenones, alkaloids and certain alkyd compounds.

For our literature review, we systematically summarized the resources, folk application, chemical composition and pharmacological activity of Cynanchum, and proposed certain suggestions according to its research status to provide reference for the comprehensive development and sustainable utilization of the species in this genus.

\section{Ethnomedicinal Uses}

According to our review on the monographs and literature, 17 medicinal plants are included in genus Cynanchum; which are C. sibiricum, C. chinense, C. auriculatum, C. officinale, C. bungei, C. otophyllum, C. corymbosum, C. amplexicaule, C. forrestii, C. stauntonii, C. vincetoxicum, C. inamoenum, CA, C. glaucescens, C. paniculatum, C. komarovii, C. versicolor, C. chekiangense and C. mooreanum. In China, plants of genus Cynanchum are mainly distributed in the southwest, northwest and northeast provinces. In local medicine, some plant roots have been used to clear away heat evil and expel superficial evils, eliminate stasis, activate blood circulation, induce diuresis and reduce edema. This review summarizes local using of Cynanchum plants in the national medicine, as shown in Table 1.

In addition, compound medication has always been an important feature of folk medicine. Cynanchum plants and other Chinese herbs are used in a number of prescriptions, such as Baiweiwan and Baiweisan. Cynanchum plants also present a long history as a folk medicine, thus providing an important reference for clinical practice (Table 2).

Table 1. Traditional use of Cynanchum species in different regions of the world.

\begin{tabular}{|c|c|c|c|}
\hline Name & Medicinal Parts & Traditional Uses & Distribution \\
\hline C. sibiricum Willd. & Whole plant & Carbuncle swollen & $\begin{array}{c}\text { Russia, China (Ovr Mongol, Gansu, } \\
\text { Xinjiang) }\end{array}$ \\
\hline C. chinense R. Br. & Whole plant & Wind-dispelling prescription & $\begin{array}{l}\text { China (Liaoning, Hebei, Henan, } \\
\text { Shandong, Shangxi, Ningxia, Gansu, } \\
\text { Jiangsu, Zhejiang) }\end{array}$ \\
\hline $\begin{array}{c}\text { C. auriculatum Royle } \\
\text { ex Wight }\end{array}$ & Roots & $\begin{array}{l}\text { Stop coughing, cure neurasthenia, } \\
\text { gastric and duodenal ulcers, } \\
\text { nephritis, and so on. }\end{array}$ & $\begin{array}{l}\text { India, China (Shandong, Hebei, Henan, } \\
\text { Shanxi, Gansu, Tibet, Anhui, Jiangsu, } \\
\text { Zhejiang, Fujian, Taiwan, Jiangxi, Hunan, } \\
\text { Hubei, Guangxi, Guangdong, Guizhou, } \\
\text { Sichuang, Yunnan) }\end{array}$ \\
\hline $\begin{array}{l}\text { C. officinale (Hemsl.) } \\
\text { Tsiang et Zhang }\end{array}$ & Roots & $\begin{array}{l}\text { Treatment of tonic analgesia, } \\
\text { epilepsy, rabies and snake bites. }\end{array}$ & $\begin{array}{c}\text { China (Shanxi, Anhui, Jiangxi, Hunan, } \\
\text { Hubei, Guangxi, Guizhou, Sichuan, } \\
\text { Yunnan) }\end{array}$ \\
\hline
\end{tabular}


Table 1. Cont.

\begin{tabular}{|c|c|c|c|}
\hline Name & Medicinal Parts & Traditional Uses & Distribution \\
\hline C. bungei Decne. & Roots & $\begin{array}{l}\text { For physically weak and insomnia, } \\
\text { forgetful dreams, skin itching. }\end{array}$ & $\begin{array}{l}\text { North Korea, China (Liaoning, } \\
\text { OvrMongol, Hubei, Hunan, Shandong, } \\
\text { Shanxi, Gansu). }\end{array}$ \\
\hline $\begin{array}{l}\text { C. otophyllum } \\
\text { Schneid. }\end{array}$ & Roots & $\begin{array}{c}\text { For rheumatoid bone pain, rubella } \\
\text { itching, epilepsy, rabies bites, snake } \\
\text { bites. }\end{array}$ & $\begin{array}{l}\text { China (Hunan, Guangxi, Guizhou, } \\
\text { Yunnan, Sichuan, Tibet) }\end{array}$ \\
\hline C. corymbosum Wight & Whole plant & $\begin{array}{l}\text { Treatment of neurasthenia, chronic } \\
\text { nephritis, orchitis, urinary } \\
\text { amenorrhea, tuberculosis, hepatitis } \\
\text { and so on. }\end{array}$ & $\begin{array}{c}\text { India, Burma, Laos, Vietnam, Kampuchea, } \\
\text { Malaysia; China (Fujian, Guangxi, } \\
\text { Guangdong, Sichuan, Yunnan) }\end{array}$ \\
\hline $\begin{array}{l}\text { C. wilfordii (Maxim.) } \\
\text { Hemsl. }\end{array}$ & Roots & $\begin{array}{l}\text { Injury, dysentery, infantile } \\
\text { malnutrition, stomach pain, } \\
\text { leucorrhea, sore ringworm. }\end{array}$ & $\begin{array}{c}\text { China (Liaoning, Henan, Shandong, } \\
\text { Shanxi, Shaanxi, Gansu, Xinjiang, Jiangsu, } \\
\text { Anhui, Sichuan, Hunan, Hubei), North } \\
\text { Korea, Japan. }\end{array}$ \\
\hline $\begin{array}{l}\text { C. amplexicaule (Sieb. } \\
\text { et Zucc.) Hemsl. var. } \\
\text { castaneum Makino }\end{array}$ & Whole plant & $\begin{array}{l}\text { Swelling and poisoning, governance } \\
\text { bruises, rheumatism. }\end{array}$ & $\begin{array}{l}\text { North Korea, Japan, China (Heilongjiang, } \\
\text { Liaoning) }\end{array}$ \\
\hline $\begin{array}{l}\text { C. forrestii Schltr. var. } \\
\text { forrestii }\end{array}$ & Roots & Reduce pain, accelerate the healing. & $\begin{array}{c}\text { Tibet, Gansu, Sichuan, Guizhou and } \\
\text { Yunnan }\end{array}$ \\
\hline $\begin{array}{l}\text { C. stauntonii (Decne.) } \\
\text { Schltr. ex Levl. }\end{array}$ & Whole plant & $\begin{array}{l}\text { Treatment of lung disease, infantile } \\
\text { malnutrition plot, cold cough and } \\
\text { chronic bronchitis and so on. }\end{array}$ & $\begin{array}{c}\text { Gansu, Anhui, Jiangsu, Zhejiang, Hunan, } \\
\text { Jiangxi, Fujian, Guangdong, Guangxi and } \\
\text { Guizhou. }\end{array}$ \\
\hline $\begin{array}{l}\text { C. vincetoxicum (L.) } \\
\text { Pers. }\end{array}$ & Roots, seeds & $\begin{array}{l}\text { Root: antiemetic; seed extract: treat } \\
\text { cardiac failure. }\end{array}$ & $\begin{array}{c}\text { China (Sichuan, Yunnan, Jiangsu and } \\
\text { Taiwan), India and central and Western } \\
\text { Europe }\end{array}$ \\
\hline $\begin{array}{l}\text { C. inamoenum } \\
\text { (Maxim.) Loes. }\end{array}$ & Roots & $\begin{array}{l}\text { Postpartum depression, pregnancy } \\
\text { enuresis, scabies and lymphadenitis. }\end{array}$ & $\begin{array}{c}\text { China (Liaoning, Hebei, Shandong, } \\
\text { Shanxi, Anhui, Zhejiang, Hubei, Hunan, } \\
\text { Shaanxi, Gansu, Guizhou, Sichuan, Tibet), } \\
\text { North Korea and Japan. }\end{array}$ \\
\hline C. atratum Bunge & Roots, stems & $\begin{array}{l}\text { Clearing heat antitoxicant, } \\
\text { insufficiency of vital energy and } \\
\text { blood, fever. }\end{array}$ & $\begin{array}{l}\text { China (Heilongjiang, Jilin, Shandong, } \\
\text { Hebei, Henan, Shanxi, Shanxi, Sichuan, } \\
\text { Guizhou, Yunnan, Guangxi, Liaoning, } \\
\text { Guangdong, Hunan, Hubei, Fujian, } \\
\text { Jiangxi, Jiangsu), North Korea and Japan }\end{array}$ \\
\hline $\begin{array}{l}\text { C. glaucesces (Decne.) } \\
\text { Hand.-Mazz. }\end{array}$ & Roots, stems & $\begin{array}{l}\text { Relieving dyspnea, antitussive and } \\
\text { antiasthmatic. }\end{array}$ & $\begin{array}{c}\text { Jiangsu, Zhejiang, Fujian, Jiangxi, Hunan, } \\
\text { Guangdong, Guangxi and Sichuan }\end{array}$ \\
\hline $\begin{array}{l}\text { C. paniculatum } \\
\text { (Bunge) Kitagawa }\end{array}$ & Roots, stems & $\begin{array}{l}\text { Rheumatism, stomach pain, } \\
\text { toothache, low back pain, flutters } \\
\text { injury, urticaria, and eczema. }\end{array}$ & $\begin{array}{l}\text { China (Liaoning, Ovr Mongol, Hebei, } \\
\text { Henan, Shanxi, Gansu, Sichuan, Guizhou, } \\
\text { Yunnan, Shandong, Anhui, Jiangsu, } \\
\text { Zhejiang, Jiangxi, Shanxi, Hubei, Hunan, } \\
\text { Guangdong and Guangxi), North Korea } \\
\text { and Japan. }\end{array}$ \\
\hline C.versicolor Bunge & Roots and stems & $\begin{array}{l}\text { Reducing fever and causing } \\
\text { diuresis, cure tuberculosis, edema, } \\
\text { pain and so on. }\end{array}$ & $\begin{array}{c}\text { China (Jilin, Liaoning, Hebei, Henan, } \\
\text { Sichuan, Shandong, Jiangsu and } \\
\text { Zhejiang) }\end{array}$ \\
\hline $\begin{array}{c}\text { C. chekiangense } \mathrm{M} \text {. } \\
\text { Cheng ex Tsiang et } \mathrm{P} \text {. } \\
\mathrm{T} \text {. Li }\end{array}$ & Roots & $\begin{array}{l}\text { Treatment of bruises, smashed } \\
\text { topical, and scabies. }\end{array}$ & $\begin{array}{l}\text { China (Zhejiang, Henan, Hunan and } \\
\text { Guangdong) }\end{array}$ \\
\hline C.mooreanum Hemsl. & Whole plant & Wash sores scabies. & $\begin{array}{l}\text { China (Henan, Hubei, Hunan, Anhui, } \\
\text { Jiangsu, Zhejiang, Jiangxi, Fujian and } \\
\text { Guangdong) }\end{array}$ \\
\hline
\end{tabular}

Note: The above information was cited from the Chinese herbal and Chinese flora. References in this table was cited from the website: http:/ / frps.eflora.cn/ and http://tool.zyy123.com/bencao/index.php. 
Table 2. Popular traditional prescription composition of Cynanchum species.

\begin{tabular}{|c|c|c|c|}
\hline Name & Compositions & Effect/Traditional Use & Ref. \\
\hline Baiwei san & $\begin{array}{c}\text { Cynanchum atratum Bunge, Zingiber officinale Rosc., Trichosanthes kirilowii Maxim., } \\
\text { Glycyrrhiza uralensis Fisch., Mirabilite. }\end{array}$ & Antidepressant & 'Qian jin yi fang', vol. 18 \\
\hline Baiwei yuan & $\begin{array}{l}\text { Cynanchum atratum Bunge, Rehmannia glutinosa (Gaetn.) Libosch. ex Fisch. et Mey., } \\
\text { Cinnamomum cassia Presl, Rubia yunnanensis Diels, Taxillus sutchuenensis (Lecomte) } \\
\text { Danser, Dendrobium nobile Lindl., Achyranthes bidentata Blume, Ligusticum chuanxiong } \\
\text { Hort., Saposhnikovia divaricata (Trucz.) Schischk., Panax ginseng C. A. Mey., } \\
\text { Aristolochia fangchi Y. C. Wu ex L. D. Chow et S. M. Hwang, Cornus officinalis Sieb. et } \\
\text { Zucc., Angelica sinensis (Oliv.) Diels, Schisandra chinensis (Turcz.) Baill. }\end{array}$ & Infertility, abortion & $\begin{array}{l}\text { 'Song.tai ping hui min he ji jv } \\
\text { fang' }\end{array}$ \\
\hline Baiwei tang & $\begin{array}{l}\text { Cynanchum atratum Bunge, Panax ginseng C. A. Mey., Angelica sinensis (Oliv.) Diels, } \\
\text { Glycyrrhiza uralensis Fisch. }\end{array}$ & $\begin{array}{l}\text { Depressed dizziness, and occurrence } \\
\text { of temporary fainting. }\end{array}$ & 'Pu ji ben shi fang', vol. 7 \\
\hline Baiwei wan & $\begin{array}{l}\text { Cynanchum atratum Bunge, Panax ginseng C. A. Mey., Aconitum carmichaelii Debx., } \\
\text { Rehmannia glutinosa (Gaetn.) Libosch. ex Fisch. et Mey., Cinnamomum cassia Presl, } \\
\text { Cynanchum otophyllum Schneid., Evodia rutaecarpa (Juss.) Benth., Angelica sinensis } \\
\text { (Oliv.) Diels, Areca catechu L. }\end{array}$ & Irregular menstruation, infertility & 'Yi lve liu shu', vol. 27 \\
\hline Baiwei gao & $\begin{array}{l}\text { Cynanchum atratum Bunge, Ampelopsis japonica (Thunb.) Makino, Bletilla striata } \\
\text { (Thunb. ex A. Murray) Rchb. f., Typhonium giganteum Engl., Angelica dahurica (Fisch. } \\
\text { ex Hoffm.) Benth. et Hook. f. ex Franch. et Sav., Paeonia lactiflora Pall., frankincense, } \\
\text { Fraxinus chinensis Roxb. }\end{array}$ & Evil sore & 'Shen hui', vol. 63 \\
\hline Baiwei shiwei wan & $\begin{array}{c}\text { Cynanchum atratum Bunge, Anemarrhena asphodeloides Bunge, Cortex Lycii, } \\
\text { Rehmannia glutinosa (Gaetn.) Libosch. ex Fisch. et Mey., Ophiopogon japonicus (L.f.) } \\
\text { Ker-Gawl., Glycyrrhiza uralensis Fisch, Dichroa febrifuga Lour., Polygonatum odoratum } \\
\text { (Mill.) Druce, Panax ginseng C. A. Mey. }\end{array}$ & Frail, afraid of cold, heat & 'Wai tai', vol. 3 \\
\hline Baiwei wan jiawei & $\begin{array}{l}\text { Saposhnikovia divaricata (Trucz.) Schischk., Notopterygium incisum Ting ex H. T. } \\
\text { Chang, Cynanchum atratum Bunge, Tribulus terrester L., pomegranate bark, } \\
\text { Taraxacum mongolicum Hand.-Mazz., Lonicera japonica Thunb. }\end{array}$ & $\begin{array}{l}\text { Breeze heat, Nasal obstruction, } \\
\text { headache, fever }\end{array}$ & 'Shen shi yao han' \\
\hline Buyi baiwei wan & $\begin{array}{l}\text { Cynanchum atratum Bunge, Dolomiaea souliei (Franch.) Shih, Angelica sinensis (Oliv.) } \\
\text { Diels, Cinnamomum cassia Presl, Lycopuslucidus Tur-Cz. var. hirtus Regel, } \\
\text { Achyranthes bidentata Blume, Rehmannia glutinosa (Gaetn.) Libosch. ex Fisch. et Mey., } \\
\text { Paeonia suffruticosa Andr., Panax ginseng C. A. Mey., Ligusticum chuanxiong Hort., } \\
\text { Atractylodes macrocephala Koidz., Citrus aurantium L., Asarum sieboldii Miq., Aconitum } \\
\text { carmichaelii Debx., Astragalus membranaceus (Fisch.) Bunge, Dipsacus asperoides C. Y. } \\
\text { Cheng et T. M. Ai, Evodia rutaecarpa (Juss.) Benth., Magnolia officinalis Rehd. et Wils. }\end{array}$ & $\begin{array}{l}\text { Postpartum weakness, pale } \\
\text { complexion, diet reduced, } \\
\text { increasingly thin. }\end{array}$ & 'Pu ji fang', vol. 350 \\
\hline
\end{tabular}


Table 2. Cont.

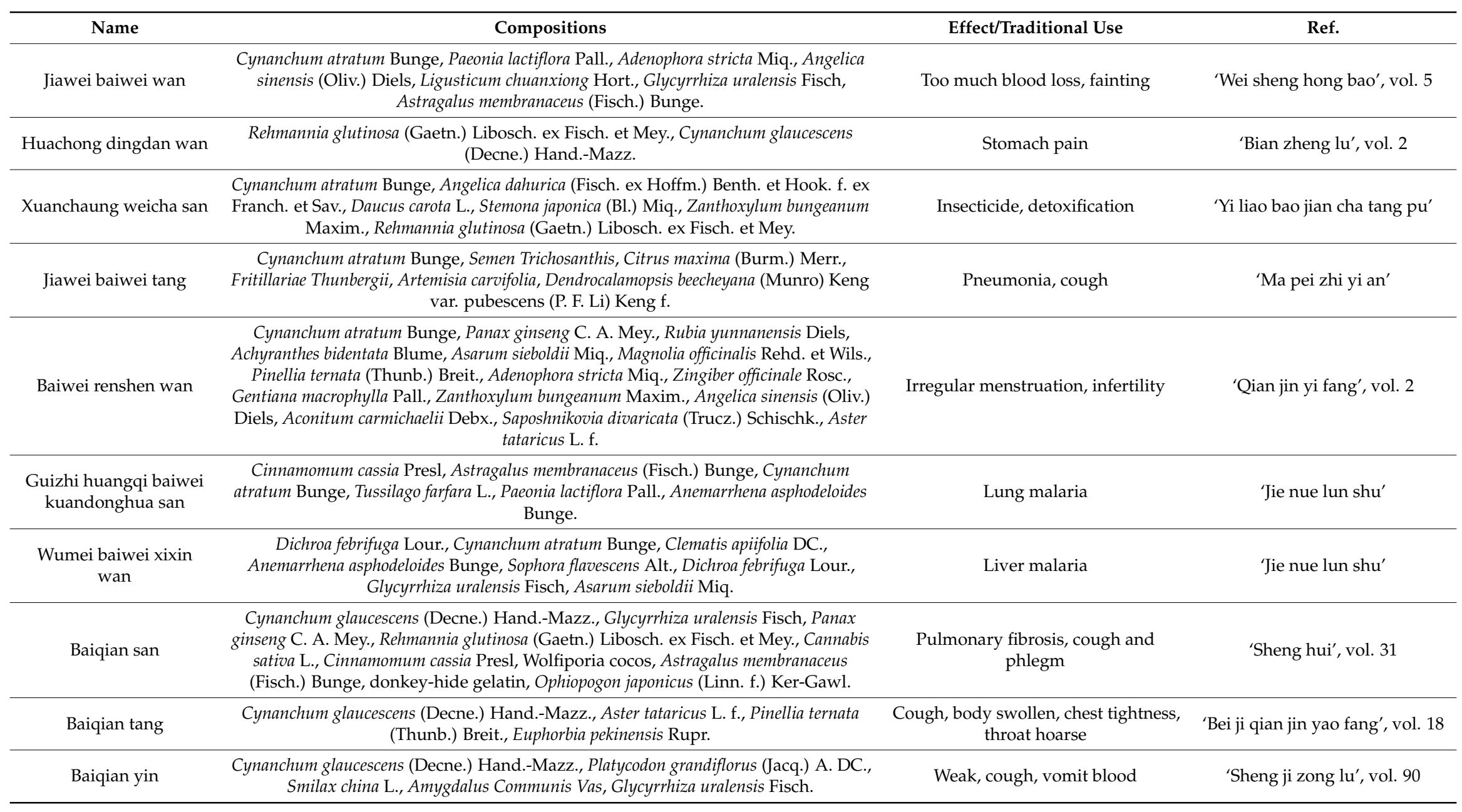


Table 2. Cont.

\begin{tabular}{|c|c|c|c|}
\hline Name & Compositions & Effect/Traditional Use & Ref. \\
\hline Shenyan baiqian tang & $\begin{array}{l}\text { Cynanchum glaucescens (Decne.) Hand.-Mazz., Pinellia ternata (Thunb.) Breit., Aster } \\
\text { tataricus L. f., Ephedra sinica Stapf, Magnolia officinalis Rehd. etWils., Panax ginseng C. } \\
\text { A. Mey., Glycyrrhiza uralensis Fisch. }\end{array}$ & $\begin{array}{l}\text { Cough, wheezing, nausea, vomiting, } \\
\text { belching, hiccups }\end{array}$ & 'Sheng ji zong lu', vol. 67 \\
\hline Xuchangqing san & $\begin{array}{l}\text { Cynanchum paniculatum (Bunge) Kitagawa, Sophora flavescens Alt., Aconitum } \\
\text { carmichaelii Debx., Evodia rutaecarpa (Juss.) Benth., Camptotheca acuminata Decne., } \\
\text { Asarum sieboldii Miq., Acorus calamus L., Pinellia ternata (Thunb.) Breit. }\end{array}$ & Scabies disease & 'Sheng ji zong lu', vol. 137 \\
\hline Xuchangqing tang & $\begin{array}{l}\text { Cynanchum paniculatum (Bunge) Kitagawa, Perotis indica (L.) Kuntze, Akebia quinata } \\
\text { (Houtt.) Decne., Malva crispa Linn., Areca catechu L., Dianthus superbus L. }\end{array}$ & $\begin{array}{l}\text { weakness of the spleen and the } \\
\text { stomach }\end{array}$ & 'Ben cao gang mu', vol. 13 \\
\hline Anwei jian & $\begin{array}{l}\text { Taraxacum mongolicum Hand.-Mazz., Cynanchum otophyllum Schneid., Glycyrrhiza } \\
\text { uralensis Fisch, Carthamus tinctorius L., Cynanchum paniculatum (Bunge) Kitagawa. }\end{array}$ & Stomach pain, blood circulation & 'Yuan zheng gang fang' \\
\hline Huainan wan & $\begin{array}{l}\text { Plantago asiatica L., Prunus salicina Lindl., Adiantum capillus-veneris L., Cynanchum } \\
\text { paniculatum (Bunge) Kitagawa. }\end{array}$ & $\begin{array}{l}\text { Tuberculosis, upset, headache and } \\
\text { vomiting }\end{array}$ & 'Pu ji fang', vol. 237 \\
\hline
\end{tabular}

References in this table was cited from the website: http:/ wwww wiki8 com. 


\section{Chemical Constituents}

At present, more than 400 compounds have been isolated from genus Cynanchum. These compounds include 388 steroids, 30 benzenes and its derivatives, 13 alkaloids, 10 flavonoids, 9 terpenes and other compounds (Table 3). The chemical structures of the primary compounds are shown in Figure 1.

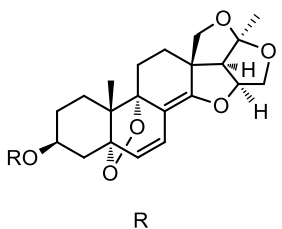

(164)<smiles>CCOC(=O)COc1cccc(OCC(=O)O)c1</smiles>

(165)

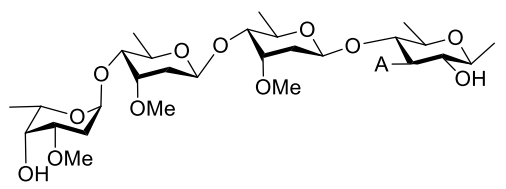

(166)

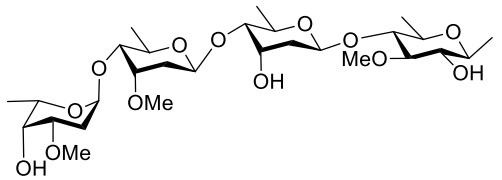

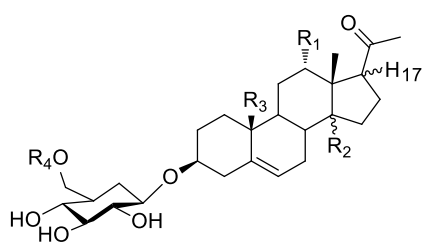

$$
\begin{aligned}
& \text { (378) } \begin{array}{ccccc}
\mathrm{R}_{1} & \mathrm{R}_{2} & \mathrm{R}_{3} & \mathrm{H}-17 & \mathrm{R}_{4}
\end{array} \\
& \text { (378) } \mathrm{OH} \quad \alpha-\mathrm{H}_{3} \quad \mathrm{CH}_{3} \quad \alpha \quad \mathrm{H} \\
& \text { (379) } \mathrm{OH} \quad \alpha-\mathrm{H} \mathrm{CH}_{3} \quad \alpha \quad \beta-D-G \mathrm{c} \\
& \text { (380) } \mathrm{OH} \quad \beta-\mathrm{OH} \mathrm{CH} \mathrm{CH}_{3} \quad \alpha \quad \mathrm{H} \\
& \text { (381) } \mathrm{OH} \quad \beta-\mathrm{OH} \mathrm{CH}_{3} \quad \alpha \quad \beta-D-G l c \\
& \text { (382) } \mathrm{H} \quad \beta-\mathrm{OH} \mathrm{CH} \mathrm{CH}_{2} \mathrm{OH} \quad \mathrm{H} \\
& \text { (383) } \mathrm{H} \quad \beta-\mathrm{OH} \mathrm{CH} \mathrm{CH}_{3} \alpha \beta-\mathrm{D}-\mathrm{GlC} \\
& \text { (384) } \mathrm{H} \quad \beta-\mathrm{OH} \mathrm{CH} \mathrm{CH}_{3} \quad \alpha \quad \mathrm{H} \\
& \text { (385) } \mathrm{H} \quad \alpha-\mathrm{Cl}_{3} \quad \alpha \quad \beta-D-G l c
\end{aligned}
$$

(370)

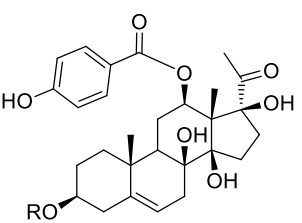

$\mathrm{R}$

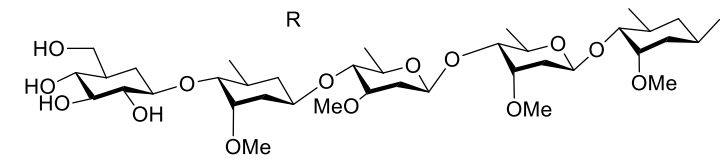

(371)

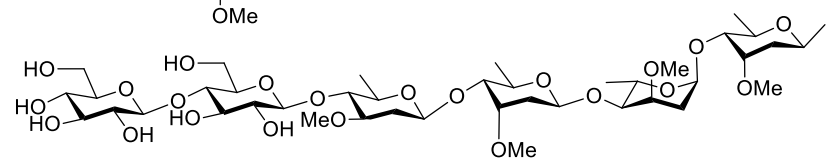

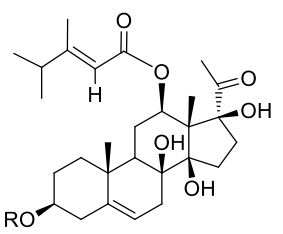

(372)

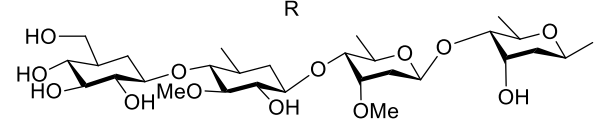

(373)

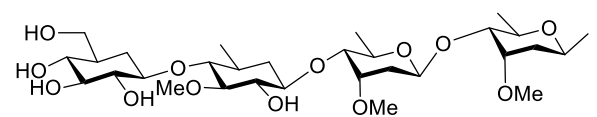

Figure 1. Structures of newly isolated C21 steroid compounds from Cynanchum species in 2016-2017.

Table 3. Compounds isolated from Cynanchum species.

\begin{tabular}{ccccc}
\hline No. & Compound Name & Species & Parts & Ref. \\
\hline \multicolumn{5}{c}{ C21 steroids } \\
\hline $\mathbf{1}$ & Cynanversicoside A & C. versicolor & Roots & {$[6]$} \\
\hline $\mathbf{2}$ & Cynanversicoside B & C. versicolor & Roots & {$[6]$} \\
\hline $\mathbf{3}$ & Cynanversicoside C & C. versicolor & Root/rhizome & {$[7]$} \\
\hline $\mathbf{4}$ & Cynanversicoside D & C. versicolor & Root/rhizome & {$[7]$} \\
\hline $\mathbf{5}$ & Cynanversicoside F & C. versicolor & Root/rhizome & {$[7]$} \\
\hline $\mathbf{6}$ & Glaucogenin B & C. glaucescens & Roots & {$[8]$} \\
\hline
\end{tabular}


Table 3. Cont.

\begin{tabular}{|c|c|c|c|c|}
\hline No. & Compound Name & Species & Parts & Ref. \\
\hline 7 & $\begin{array}{l}\text { 12 } \beta \text {-O-(4-hydroxybenzoyl)- } 8 \beta, 14 \beta, 17 \beta- \\
\text { trihydroxypregn-2,5-diene-20-one }\end{array}$ & C. wilfordii & Roots & [9] \\
\hline 8 & $\begin{array}{c}\text { 12 } \beta \text {-O-benzoyl- } 8 \beta, 14 \beta, 17 \beta \text { - } \\
\text { trihydroxypregn-2,5-diene-20-one }\end{array}$ & C. wilfordii & Roots & [9] \\
\hline 9 & Glaucoside A & C. glaucescens & Roots & [8] \\
\hline 10 & Glaucoside B & C. glaucescens & Roots & [8] \\
\hline 11 & Glaucoside C & C. glaucescens & Roots & [10] \\
\hline 12 & Glaucoside D & C. glaucescens & Roots & [8] \\
\hline 13 & Glaucoside E & C. glaucescens & Roots & [8] \\
\hline 14 & Glaucoside F & C. glaucescens & Roots & [8] \\
\hline 15 & Glaucoside G & C. glaucescens & Roots & [8] \\
\hline 16 & Glaucoside H & C. glaucescens & Roots & [8] \\
\hline 17 & Glaucoside I & C. glaucescens & Roots & [8] \\
\hline 18 & Glaucoside J & C. glaucescens & Roots & [8] \\
\hline 19 & Cynatratoside F & C. atratum & Roots & [8] \\
\hline 20 & Cynatratoside C & C. atratum & Roots & [10] \\
\hline 21 & Cynatratoside A & C. atratum & Roots & [11] \\
\hline 22 & Cynatratoside B & C. atratum & Roots & [12] \\
\hline 23 & Atratoside A & C. atratum & Roots & [13] \\
\hline 24 & Atratoside B & C. atratum & Roots & [13] \\
\hline 25 & Atratoside C & C. atratum & Roots & [14] \\
\hline 26 & Atratoside D & C. atratum & Roots & [8] \\
\hline 27 & Otophylloside A & $\begin{array}{l}\text { C. forrestii } \\
\text { C. otophyllum } \\
\text { C. wallichii }\end{array}$ & Roots & [15] \\
\hline 28 & Otophylloside B & $\begin{array}{l}\text { C. forrestii } \\
\text { C. otophyllum } \\
\text { C. wallichii }\end{array}$ & Roots & [15] \\
\hline 29 & Otophylloside C & C. otophyllum & Roots & [16] \\
\hline 30 & Otophylloside F & C. otophyllum & Roots & [16] \\
\hline 31 & Otophylloside H & C. otophyllum & Roots & [17] \\
\hline 32 & Otophylloside I & C. otophyllum & Roots & [17] \\
\hline 33 & Otophylloside J & C. otophyllum & Roots & [17] \\
\hline 34 & Otophylloside K & C. otophyllum & Roots & [17] \\
\hline 35 & Otophylloside L & $\begin{array}{l}\text { C. otophyllum } \\
\text { C. auriculatum }\end{array}$ & Roots & [17] \\
\hline 36 & Otophylloside M & C. otophyllum & Roots & [17] \\
\hline 37 & Otophylloside $\mathrm{N}$ & C. forrestii & Roots & [15] \\
\hline 38 & Otophylloside O & C. forrestii & Roots & [15] \\
\hline 39 & Otophylloside P & C. forrestii & Roots & [15] \\
\hline 40 & Otophylloside Q & C. forrestii & Roots & [15] \\
\hline
\end{tabular}


Table 3. Cont.

\begin{tabular}{|c|c|c|c|c|}
\hline No. & Compound Name & Species & Parts & Ref. \\
\hline 41 & Otophylloside R & C. forrestii & Roots & [15] \\
\hline 42 & Otophylloside S & C. forrestii & Roots & [15] \\
\hline 43 & Otophylloside T & C. otophyllum & Roots & [16] \\
\hline 44 & Otophylloside U & C. otophyllum & Roots & [18] \\
\hline 45 & Otophylloside V & C. otophyllum & Roots & [18] \\
\hline 46 & Otophylloside W & C. otophyllum & Roots & [18] \\
\hline 47 & Sibiricoside D & C. sibiricum & Roots & [19] \\
\hline 48 & Sibiricoside E & C. sibiricum & Roots & [19] \\
\hline 49 & Sibirigenin & C. sibiricum & Roots & [20] \\
\hline 50 & Penupogenin & C. sibiricum & Roots & [20] \\
\hline 51 & $\begin{array}{l}\text { Penupogenin3-O- } \beta \text {-D-glucopyranosyl- } \\
(1 \rightarrow 4) \text { - } \beta \text {-l-cymaropyranosyl- }(1 \rightarrow 4)-\beta \text {-D- } \\
\text { cymaropyranosyl-( }(1 \rightarrow 4) \text { - } \alpha \text {-L-diginopyranosyl- } \\
(1 \rightarrow 4)-\beta \text {-D-cymaropyranoside }\end{array}$ & C. bungei & Stems & [21] \\
\hline 52 & Cynanoside A & C. atratum & Roots & [22] \\
\hline 53 & Cynanoside B & C. atratum & Roots & [22] \\
\hline 54 & Cynanoside C & C. atratum & Roots & [22] \\
\hline 55 & Cynanoside D & C. atratum & Roots & [22] \\
\hline 56 & Cynanoside E & C. atratum & Roots & [22] \\
\hline 57 & Cynanoside F & C. atratum & Roots & {$[22]$} \\
\hline 58 & Cynanoside G & C. atratum & Roots & [22] \\
\hline 59 & Cynanoside $\mathrm{H}$ & C. atratum & Roots & {$[22]$} \\
\hline 60 & Cynanoside I & $\begin{array}{l}\text { C. atratum } \\
\text { C. versicolor }\end{array}$ & Roots & [22] \\
\hline 61 & Cynanoside J & C. atratum & Roots & [22] \\
\hline 62 & Cynanoside $\mathrm{K}$ & C. atratum & Roots & [13] \\
\hline 63 & Cynanoside L & C. atratum & Roots & [13] \\
\hline 64 & Cynanoside $\mathrm{M}$ & C. atratum & Roots & [13] \\
\hline 65 & Cynanoside $\mathrm{N}$ & C. atratum & Roots & [13] \\
\hline 66 & Cynanoside $\mathrm{O}$ & C. atratum & Roots & [13] \\
\hline 67 & Cynanosides $\mathrm{P}_{1}$ & C. atratum & Roots & [14] \\
\hline 68 & Cynanosides $\mathrm{P}_{2}$ & C. atratum & Roots & [14] \\
\hline 69 & Cynanosides $\mathrm{P}_{3}$ & C. atratum & Roots & [14] \\
\hline 70 & Cynanosides $\mathrm{P}_{4}$ & C. atratum & Roots & [14] \\
\hline 71 & Cynanosides $\mathrm{P}_{5}$ & C. atratum & Roots & [14] \\
\hline 72 & Cynanosides $\mathrm{Q}_{1}$ & C. atratum & Roots & [14] \\
\hline 73 & Cynanosides $\mathrm{Q}_{2}$ & C. atratum & Roots & [14] \\
\hline 74 & Cynanosides $Q_{3}$ & C. atratum & Roots & [14] \\
\hline 75 & Cynanosides $\mathrm{R}_{1}$ & C. atratum & Roots & [14] \\
\hline 76 & Cynanosides $R_{2}$ & C. atratum & Roots & [14] \\
\hline 77 & Cynanosides $R_{3}$ & C. atratum & Roots & [14] \\
\hline 78 & Cynanoside S & C. atratum & Roots & [14] \\
\hline
\end{tabular}


Table 3. Cont.

\begin{tabular}{|c|c|c|c|c|}
\hline No. & Compound Name & Species & Parts & Ref. \\
\hline 79 & Sublanceoside $\mathrm{E}_{3}$ & C. atratum & Roots & [14] \\
\hline 80 & Chekiangensoside A & C. chekiangense & Roots & [23] \\
\hline 81 & Chekiangensoside B & C. chekiangense & Roots & [23] \\
\hline 82 & Chekiangensoside C & C. chekiangense & Roots & [14] \\
\hline 83 & Chekiangensoside D & C. chekiangense & Roots & [24] \\
\hline 84 & Chekiangensoside E & C. chekiangense & Roots & [24] \\
\hline 85 & Cynatroside A & C. atratum & Roots & [25] \\
\hline 86 & Cynatroside B & C. atratum & Roots & [14] \\
\hline 87 & Cynatroside C & C. atratum & Roots & [25] \\
\hline 88 & Wilfoside A & C. wilfordii & Roots & [26] \\
\hline 89 & Wilfoside B & C. wilfordii & Roots & [26] \\
\hline 90 & Wilfoside C & C. wilfordii & Roots & [26] \\
\hline 91 & Wilfoside D & C. wilfordii & Roots & [26] \\
\hline 92 & Wilfoside E & C. wilfordii & Roots & [26] \\
\hline 93 & Wilfoside F & C. wilfordii & Roots & [26] \\
\hline 94 & Wilfoside G & C. wilfordii & Roots & [26] \\
\hline 95 & Wilfoside $\mathrm{H}$ & C. wilfordii & Roots & [26] \\
\hline 96 & Wilfoside KIN & C.wilfordii & Roots & [26] \\
\hline 97 & Wilfoside $\mathrm{K}_{1} \mathrm{GG}$ & C. wilfordii & Roots & [27] \\
\hline 98 & Wilfoside $\mathrm{C}_{1} \mathrm{GG}$ & C. wilfordii & Roots & [27] \\
\hline 99 & Wilfoside $\mathrm{C}_{1} \mathrm{~N}$ & C. taiwanianum & Roots & [28] \\
\hline 100 & Wilfoside $\mathrm{C}_{2} \mathrm{~N}$ & C. taiwanianum & Roots & [28] \\
\hline 101 & Wilfoside $\mathrm{C}_{3} \mathrm{~N}$ & C. auriculatum & Roots & [29] \\
\hline 102 & Wilfoside $\mathrm{M}_{1} \mathrm{~N}$ & C. auriculatum & Roots & [30] \\
\hline 103 & Wilfoside $\mathrm{C}_{1} \mathrm{G}$ & C. auriculatum & Roots & [30] \\
\hline 104 & Wilfoside $\mathrm{C}_{2} \mathrm{G}$ & C. otophyllum & Roots & [31] \\
\hline 105 & Amplexicoside A & C. amplexicaule & Roots & [32] \\
\hline 106 & Amplexicoside B & C. amplexicaule & Roots & [32] \\
\hline 107 & Amplexicoside C & C. amplexicaule & Roots & [32] \\
\hline 108 & Amplexicoside D & C. amplexicaule & Roots & [32] \\
\hline 109 & Amplexicoside E & C. amplexicaule & Roots & [32] \\
\hline 110 & Amplexicoside F & C. amplexicaule & Roots & [32] \\
\hline 111 & Amplexicoside G & C. amplexicaule & Roots & [32] \\
\hline 112 & Tylophoside A & C. amplexicaule & Roots & [32] \\
\hline 113 & Hancoside A & $\begin{array}{l}\text { C. amplexicaule } \\
\text { C. komarovii }\end{array}$ & Roots & [33] \\
\hline 114 & Hancoside & $\begin{array}{c}\text { C. forrestii } \\
\text { C. hunmkiunum }\end{array}$ & Roots & [34] \\
\hline 115 & Neocynapanogenin F 3-O- $\beta$-D-thevetoside & C. paniculatum & Roots & [35] \\
\hline 116 & Neocynapanogenin F & C. paniculatum & Roots & [35] \\
\hline
\end{tabular}


Table 3. Cont.

\begin{tabular}{|c|c|c|c|c|}
\hline No. & Compound Name & Species & Parts & Ref. \\
\hline 117 & Neocynapanogenin F 3-O- $\beta$-D-thevetopyranoside & C. atratum. & Roots & [36] \\
\hline 118 & Glaucogenin C & $\begin{array}{l}\text { C. hunmkiunum } \\
\text { C. atratum }\end{array}$ & Roots & [37] \\
\hline 119 & $\begin{array}{c}\text { Glaucogenin } C \\
\text { 3-O- } \alpha \text {-L-cymaropyranosyl- }(1 \rightarrow 4)-\beta \text {-D- } \\
\text { digitoxopyranosyl- }(1 \rightarrow 4)-\beta \text {-D-canaropyranoside }\end{array}$ & C. stauntonii & root & [38] \\
\hline 120 & $\begin{array}{c}\text { Glaucogenin C } \\
\text { 3-O- } \beta \text {-D-cymaropyranosyl- }(1 \rightarrow 4)-\alpha \text {-L- } \\
\text { diginopyranosyl- }(1 \rightarrow 4)-\beta \text {-D-thevetopyranoside }\end{array}$ & C. atratum & Roots & [39] \\
\hline 121 & Glaucogenin C 3-O- $\beta$-D-thevetopyranoside & C. atratum & Roots & [39] \\
\hline 122 & Glaucogenin C mono-D-thevetoside & C. stauntonii & Roots & [40] \\
\hline 123 & Glaucogenin C 3-O- $\beta$-D-oleandropyranoside & C. atratum. & Roots & [36] \\
\hline 124 & $\begin{array}{c}\text { Glaucogenin C 3-O- } \alpha \text {-L-diginopyranosyl-(1 } \rightarrow 4) \text { - } \\
\beta \text {-D-thevetopyranoside }\end{array}$ & C. atratum. & Roots & [36] \\
\hline 125 & $\begin{array}{c}\text { Glaucogenin C } \\
\text { 3-O- } \alpha \text {-L-cymaropyranosyl-( }(1 \rightarrow 4)-\beta \text {-D- } \\
\text { cymaropyranosyl- }(1 \rightarrow 4)-\beta \text {-D-oleandropyranoside }\end{array}$ & C. atratum. & Roots & [36] \\
\hline 126 & $\begin{array}{c}\text { Glaucogenin C } \\
\text { 3-O- } \alpha \text {-L-cymaropyranosyl- }(1 \rightarrow 4)-\beta \text {-D- } \\
\text { digitoxopyranosy- }(1 \rightarrow 4)-\beta \text {-D-oleandropyranoside }\end{array}$ & C. atratum. & Roots & [36] \\
\hline 127 & $\begin{array}{c}\text { Glaucogenin C } \\
\text { 3-O- } \beta \text {-D-cymaropyranosyl- }(1 \rightarrow 4)-\alpha \text {-L- } \\
\text { diginopyranosyl- }(1 \rightarrow 4)-\beta \text {-D-cymaropyranoside }\end{array}$ & C. atratum. & Roots & [36] \\
\hline 128 & $\begin{array}{c}\text { Glaucogenin } \mathrm{C} \\
\text { 3-O- } \alpha \text {-D-oleandropyranosyl-( }(1 \rightarrow 4)-\beta \text {-D- } \\
\text { digitoxopyranosyl-(1 }(1 \rightarrow 4)-\beta \text {-D-oleandropyranoside }\end{array}$ & C. atratum. & Roots & [36] \\
\hline 129 & Glaucogenin C 3-O- $\beta$-D-thevetoside & C. paniculatum. & Root/rhizome & [41] \\
\hline 130 & Glaucogenin A & C. atratum. & Roots & [36] \\
\hline 131 & Glaucogenin A 3-O- $\beta$-D-oleandropyranoside & C. atratum. & Roots & [36] \\
\hline 132 & Glaucogenin A 3-O- $\beta$-D-digitoxopyranoside & C. atratum. & Roots & [36] \\
\hline 133 & $\begin{array}{l}\text { Glaucogenin } \mathrm{A} \\
\text { 3-O- } \beta \text {-D-digitoxopyranosyl-( }(1 \rightarrow 4)-\beta \text {-D } \\
\text { cymaropyranoside }\end{array}$ & C. atratum. & Roots & [36] \\
\hline 134 & $\begin{array}{l}\text { Glaucogenin A 3-O- } \beta \text {-D-glucopyranosyl- } \\
\quad(1 \rightarrow 4)-\beta \text {-D-oleandropyranoside }\end{array}$ & C. atratum. & Roots & [36] \\
\hline 135 & $\begin{array}{l}\text { Glaucogenin A 3-O- } \beta \text {-D- } \\
\text { cymaropyranosyl- }(1 \rightarrow 4)-\alpha \text {-L-diginopyranosyl- } \\
(1 \rightarrow 4)-\beta \text {-D-cymaropyranoside }\end{array}$ & C. atratum. & Roots & [36] \\
\hline 136 & $\begin{array}{l}\text { Glaucogenin A 3-O- } \alpha \text {-L- } \\
\text { cymaropyranosyl- }(1 \rightarrow 4) \text { - } \beta \text {-D-digitoxopyranosyl- } \\
(1 \rightarrow 4)-\beta \text {-D-cymaropyranoside }\end{array}$ & C. atratum. & Roots & [36] \\
\hline 137 & $\begin{array}{l}\text { Glaucogenin A 3-O- } \alpha \text {-D- } \\
\text { oleandropyranosyl- }(1 \rightarrow 4)-\beta \text {-D-digitoxopyranosyl- } \\
(1 \rightarrow 4)-\beta \text {-D-oleandropyranoside }\end{array}$ & C. atratum. & Roots & [36] \\
\hline 138 & $\begin{array}{l}\text { Glaucogenin A 3-O- } \alpha \text {-L- } \\
\text { cymaropyranosyl- }(1 \rightarrow 4)-\beta \text {-D-digitoxopyranosyl- } \\
(1 \rightarrow 4)-\beta \text {-D-digitoxopyranoside }\end{array}$ & C. atratum. & Roots & [36] \\
\hline
\end{tabular}


Table 3. Cont.

\begin{tabular}{|c|c|c|c|c|}
\hline No. & Compound Name & Species & Parts & Ref. \\
\hline 139 & $\begin{array}{l}\text { Glaucogenin A 3-O- } \beta \text {-D- } \\
\text { oleandropyranosyl- }(1 \rightarrow 4)-\beta \text {-D-digitoxopyranosyl- } \\
(1 \rightarrow 4)-\beta \text {-D-oleandropyranoside }\end{array}$ & C. atratum. & Roots & [36] \\
\hline 140 & $\begin{array}{l}\text { Glaucogenin A 3-O- } \alpha \text {-L- } \\
\text { cymaropyranosyl- }(1 \rightarrow 4)-\beta \text {-D-cymaropyranosyl- } \\
(1 \rightarrow 4)-\beta \text {-D-oleandropyranoside }\end{array}$ & C. atratum. & Roots & [36] \\
\hline 141 & $\begin{array}{l}\text { Glaucogenin A 3-O- } \alpha \text {-L- } \\
\text { cymaropyranosyl- }(1 \rightarrow 4)-\beta \text {-D-cymaropyranosyl- } \\
(1 \rightarrow 4)-\beta \text {-D-cymaropyranoside }\end{array}$ & C. atratum. & Roots & [36] \\
\hline 142 & $\begin{array}{c}\text { Glaucogenin A 3-O- } \beta \text {-D- } \\
\text { glucopyranosyl- }(1 \rightarrow 4)-\beta \text {-D-glucopyranosyl- } \\
(1 \rightarrow 4)-\beta \text {-D-oleandropyranoside }\end{array}$ & C. atratum. & Roots & [36] \\
\hline 143 & $\begin{array}{l}\text { Glaucogenin A 3-O- } \alpha \text {-L- } \\
\text { oleandropyranosyl- }(1 \rightarrow 4)-\beta \text {-D-digitoxopyranosyl- } \\
(1 \rightarrow 4)-\beta \text {-D-oleandropyranoside }\end{array}$ & C. atratum. & Roots & [36] \\
\hline 144 & Glaucogenin D & C. paniculatum. & Root /rhizome & [41] \\
\hline 145 & Stauntoside A & C. stauntoi & Roots & [42] \\
\hline 146 & Stauntoside B & C. stauntoi & Roots & [42] \\
\hline 147 & Stauntoside C & C. stauntonii & Roots & [43] \\
\hline 148 & Stauntoside D & C. stauntonii & Roots & [43] \\
\hline 149 & Stauntoside E & C. stauntonii & Roots & [43] \\
\hline 150 & Stauntoside F & C. stauntonii & Roots & [43] \\
\hline 151 & Stauntoside G & C. stauntonii & Roots & [43] \\
\hline 152 & Stauntoside $\mathrm{H}$ & C. stauntonii & Roots & [43] \\
\hline 153 & Stauntoside I & C. stauntonii & Roots & [43] \\
\hline 154 & Stauntoside J & C. stauntonii & Roots & [43] \\
\hline 155 & Stauntoside K & C. stauntonii & Roots & [43] \\
\hline 156 & Stauntoside L & C. stauntonii & Roots & [44] \\
\hline 157 & Stauntoside M & C. stauntonii & Roots & [44] \\
\hline 158 & Stauntoside $\mathrm{O}$ & C. stauntonii & Roots & [44] \\
\hline 159 & Stauntoside $\mathrm{P}$ & C. stauntonii & Roots & [44] \\
\hline 160 & Stauntoside Q & C. stauntonii & Roots & [44] \\
\hline 161 & Stauntoside R & C. stauntonii & Roots & [44] \\
\hline 162 & Stauntoside S & C. stauntonii & Roots & [44] \\
\hline 163 & Stauntoside $\mathrm{T}$ & C. stauntonii & Roots & [44] \\
\hline 164 & Stauntoside UA & C. stauntonii . & Roots & [45] \\
\hline 165 & Stauntoside $\mathrm{UA}_{1}$ & C. stauntonii. & Roots & [45] \\
\hline 166 & Stauntoside $\mathrm{UA}_{2}$ & C. stauntonii . & Roots & [45] \\
\hline 167 & Kidjoranin & $\begin{array}{l}\text { C. wilfordii. } \\
\text { C. auriculatum }\end{array}$ & Roots & [9] \\
\hline 168 & $\begin{array}{c}\text { Kidjoranin-3-O- } \beta \text {-D-oleandropyranosyl-( }(1 \rightarrow 4) \text { - } \\
\beta \text {-D-oleandropyranosyl-( }(1 \rightarrow 4)-\beta \text {-D- } \\
\text { cymaropyranosyl-( }(1 \rightarrow 4) \text { - } \beta \text {-D-cymaropyranoside }\end{array}$ & C. otophyllum & Roots & [46] \\
\hline 169 & Kidjoranin 3-O- $\beta$-D-digitoxopyranoside & C. otophyllum & Roots & [47] \\
\hline 170 & 20-O-(4-hydroxybenzoyl)-kidjoranin & C. wilfordii & Roots & [9] \\
\hline
\end{tabular}


Table 3. Cont.

\begin{tabular}{|c|c|c|c|c|}
\hline No. & Compound Name & Species & Parts & Ref. \\
\hline 171 & 20-O-vanilloyl-kidjoranin & C. wilfordii & Roots & [9] \\
\hline 172 & 20-O-salicyl-kidjoranin & C. wilfordii & Roots & [9] \\
\hline 173 & 20-O-(4-hydroxybenzoyl)-kidjoranin & C. wilfordii. & Roots & [9] \\
\hline 174 & $\begin{array}{l}\text { 12 } \beta \text {-O-(4-hydroxybenzoyl)- } 8 \beta, 14 \beta, 17 \beta- \\
\text { trihydroxypregn-2,5-diene-20-one }\end{array}$ & C. wilfordii. & Roots & [9] \\
\hline 175 & Caudatin & C. auriculatum & Roots & [29] \\
\hline 176 & $\begin{array}{l}\text { caudatin-2,6-dideoxy-3-O-methy- } \beta \text { - } \\
\text { D-cymaropyranoside }\end{array}$ & C. auriculatum & Roots & [48] \\
\hline 177 & 3-O-methyl-caudatin & C. wilfordii & Roots & [9] \\
\hline 178 & $\begin{array}{c}\text { Caudatin 3-O- } \beta \text {-D-glucopyranosyl- } \\
(1 \rightarrow 4) \text { - } \beta \text {-D-oleandropyranosyl- }(1 \rightarrow 4)-\beta \text {-D- } \\
\text { cymaropyranosyl- }(1 \rightarrow 4)-\beta \text {-D-cymaropyranoside }\end{array}$ & C. forrestii & Roots & [15] \\
\hline 179 & $\begin{array}{c}\text { Caudatin 3-O- } \alpha \text {-L-cymaropyranosyl- } \\
(1 \rightarrow 4)-\alpha \text {-D-oleandropyranosyl- }(1 \rightarrow 4)-\alpha \text {-L- } \\
\text { cymaropyranosyl- }(1 \rightarrow 4) \text { - } \beta \text {-D-glucopyranosyl- } \\
(1 \rightarrow 4)-\alpha \text {-D-oleandropyranosyl- }(1 \rightarrow 4)-\beta \text {-D- } \\
\text { oleandropyranosyl- }(1 \rightarrow 4)-\beta \text {-D-diginopyranoside }\end{array}$ & C. otophyllum & Rhizome & [49] \\
\hline 180 & $\begin{array}{c}\text { Caudatin } 3-O-\beta \text {-D-cymaropyranosyl- } \\
(1 \rightarrow 4)-\alpha \text {-D-oleandropyranosyl- }(1 \rightarrow 4)-\alpha \text {-L- } \\
\text { cymaropyranosyl- }(1 \rightarrow 4)-\beta \text {-D-glucopyranosyl- } \\
(1 \rightarrow 4)-\beta \text {-D-oleandropyranosyl- }(1 \rightarrow 4)-\beta \text {-D- } \\
\text { cymaropyranosyl-( }(1 \rightarrow 4)-\beta \text {-D-diginopyranoside }\end{array}$ & C. otophyllum & Rhizome & [49] \\
\hline 181 & $\begin{array}{l}\text { Caudatin 3-O- } \beta \text {-D-cymaropyranosyl- } \\
(1 \rightarrow 4)-\beta \text {-D-cymaropyranoside }\end{array}$ & C. otophyllum & Roots & [16] \\
\hline 182 & $\begin{array}{c}\text { Caudatin 3-O- } \beta \text {-D-glucopyranosyl- } \\
(1 \rightarrow 4) \text { - } \beta \text {-D-oleandropyranosyl- }(1 \rightarrow 4)-\beta \text {-D- } \\
\text { cymaropyranosyl- }(1 \rightarrow 4)-\beta \text {-D-cymaropyranoside }\end{array}$ & C. otophyllum & Roots & [16] \\
\hline 183 & $\begin{array}{c}\text { Caudatin 3-O- } \beta \text {-D-glucopyranosyl- } \\
(1 \rightarrow 4)-\beta \text {-D-cymaropyranosyl- }(1 \rightarrow 4)-\alpha \text {-L- } \\
\text { diginopyranosyl- }(1 \rightarrow 4) \text { - } \beta \text {-D-cymaropyranoside }\end{array}$ & C. wilfordii. & Roots & [50] \\
\hline 184 & $\begin{array}{c}\text { Caudatin 3-O- } \beta \text {-D-cymaropyranosyl- } \\
(1 \rightarrow 4)-\beta \text {-D-oleandropyranosyl- }(1 \rightarrow 4) \\
\text { - } \beta \text {-D-cymaropyranoside }\end{array}$ & C. otophyllum & Roots & [46] \\
\hline 185 & $\begin{array}{c}\text { Caudatin-3-O- } \beta \text {-D-oleandropyranosyl- }(1 \rightarrow 4) \\
-\beta \text {-D-thevetopyranosyl- }(1 \rightarrow 4)-\beta \text {-D-cymaropyranoside }\end{array}$ & C. otophyllum & Roots & [46] \\
\hline 186 & $\begin{array}{l}\text { Caudatin-3-O- } \beta \text {-D-thevetopyranosyl- }(1 \rightarrow 4) \\
-\beta \text {-D-cymaropyranosyl-( }(1 \rightarrow 4)-\beta \text {-D-cymaropyranoside }\end{array}$ & C. otophyllum & Roots & [46] \\
\hline 187 & $\begin{array}{l}\text { Caudatin-3-O- } \beta \text {-D-thevetopyranosyl- }(1 \rightarrow 4) \\
\text { - } \beta \text {-D-cymaropyranosyl- }(1 \rightarrow 4)-\beta \text {-D-digitoxopyranoside. }\end{array}$ & C. otophyllum & Roots & [46] \\
\hline 188 & $\begin{array}{c}\text { Caudatin-3-O- } \beta \text {-D-cymaropyranosyl- }(1 \rightarrow 4) \\
\text { - } \beta \text {-D-oleandropyranosyl- }(1 \rightarrow 4)-\beta \text {-D- } \\
\text { cymaropyranosyl- }(1 \rightarrow 4) \text { - } \beta \text {-D-digitoxopyranoside }\end{array}$ & C. otophyllum & Roots & [46] \\
\hline 189 & $\begin{array}{c}\text { Caudatin-3-O- } \alpha \text {-L-cymaropyranosyl- }(1 \rightarrow 4) \\
-\alpha \text {-D-cymaropyranosyl- }(1 \rightarrow 4)-\alpha \text { - }- \\
\text { cymaropyranosyl- }(1 \rightarrow 4)-\beta \text {-D-digitoxopyranoside }\end{array}$ & C. otophyllum & Roots & [46] \\
\hline 190 & $\begin{array}{c}\text { Caudatin 3-O- } \beta \text {-D-oleandropyranosyl- }(1 \rightarrow 4) \\
-\beta \text {-D-thevetopyranosyl-( }(1 \rightarrow 4)-\beta \text {-D- } \\
\text { cymaropyranosyl- }(1 \rightarrow 4)-\beta \text {-D-cymaropyranoside }\end{array}$ & C. otophyllum & Roots & [46] \\
\hline
\end{tabular}


Table 3. Cont.

\begin{tabular}{|c|c|c|c|c|}
\hline No. & Compound Name & Species & Parts & Ref. \\
\hline 191 & $\begin{array}{c}\text { Caudatin 3-O- } \alpha \text {-L-cymaropyranosyl- } \\
(1 \rightarrow 4)-\beta \text {-D-cymaropyranosyl- }(1 \rightarrow 4)-\alpha \text {-L- } \\
\text { cymaropyranosyl-( }(1 \rightarrow 4)-\beta \text {-D-cymaropyranosyl- } \\
(1 \rightarrow 4) \text { - } \beta \text {-D-digitoxopyranoside. }\end{array}$ & C. otophyllum & Roots & [46] \\
\hline 192 & $\begin{array}{c}\text { Caudatin 3-O- } \alpha \text {-L-cymaropyranosyl- } \\
(1 \rightarrow 4)-\beta \text {-D-cymaropyranosyl- }(1 \rightarrow 4)-\beta \text {-D- } \\
\text { cymaropyranosyl- }(1 \rightarrow 4) \text { - } \alpha \text {-L-cymaropyranosyl- } \\
(1 \rightarrow 4)-\beta \text {-D-oleandropyranosyl- }(1 \rightarrow 4)-\beta \text { - } \\
\text { D-cymaropyranoside }\end{array}$ & C. otophyllum & Roots & [46] \\
\hline 193 & $\begin{array}{c}\text { Caudatin 3-O- } \beta \text {-D-cymaropyranosyl- } \\
(1 \rightarrow 4)-\beta \text {-D-oleandropyranosyl- }(1 \rightarrow 4)-\beta \text {-D- } \\
\text { cymaropyranosyl-( }(1 \rightarrow 4) \text { - } \beta \text {-D-cymaropyranoside }\end{array}$ & C. otophyllum & Roots & [46] \\
\hline 194 & $\begin{array}{l}\text { Caudatin 3-O- } \beta \text {-D-oleandropyranosyl- } \\
(1 \rightarrow 4)-\beta \text {-D-digitoxopyranosyl- }(1 \rightarrow 4)-\beta \text {-D- } \\
\text { cymaropyranoside }\end{array}$ & C. otophyllum & Roots & [46] \\
\hline 195 & $\begin{array}{c}\text { Caudatin 3-O- } \beta \text {-D-oleandropyranosyl- } \\
(1 \rightarrow 4) \text { - } \beta \text {-D-cymaropyranosyl- }(1 \rightarrow 4)- \\
\beta \text {-D-digitoxopyranoside }\end{array}$ & C. otophyllum & Roots & [46] \\
\hline 196 & Caudatin 3- $\beta$-D-digitoxopyranoside & C. otophyllum & Roots & [47] \\
\hline 197 & $\begin{array}{l}\text { Caudatin 3-O- } \alpha \text {-L-diginopyranosyl- } \\
(1 \rightarrow 4) \text { - } \beta \text {-D-cymaropyranoside }\end{array}$ & C. otophyllum & Roots & [47] \\
\hline 198 & $\begin{array}{c}\text { Caudatin 3-O- } \beta \text {-D-glucopyranosyl- } \\
(1 \rightarrow 4) \text { - } \beta \text {-D-digitoxopyranosyl- }(1 \rightarrow 4)-\beta \text {-D- } \\
\text { diginopyranosyl-( }(1 \rightarrow 4) \text { - } \alpha \text {-D-oleandropyranoside }\end{array}$ & C. otophyllum & Rhizome & [51] \\
\hline 199 & $\begin{array}{c}\text { Caudatin3-O- } \beta \text {-D-oleandropyranosyl- }(1 \rightarrow 4) \\
-\alpha \text {-D-oleandropyranosyl- }(1 \rightarrow 4)-\alpha- \\
\text { D-oleandropyranoside }\end{array}$ & C. otophyllum & Rhizome & [51] \\
\hline 200 & $\begin{array}{c}\text { Caudatin3-O- } \beta \text {-D-glucopyranosyl-( }(1 \rightarrow 4) \\
-\alpha \text {-D-oleandropyranosyl- }(1 \rightarrow 4)-\beta \text {-D- } \\
\text { diginopyranosyl-( }(1 \rightarrow 4)-\alpha \text {-D-oleandropyranoside }\end{array}$ & C. otophyllum & Rhizome & [51] \\
\hline 201 & Qingyangshengenin & C. wilfordii. & Roots & [9] \\
\hline 202 & $\begin{array}{l}\text { Qingyangshengenin 3-O- } \beta \text {-D-cymaropyranosyl- } \\
\qquad(1 \rightarrow 4) \text { - } \beta \text {-D-digitoxopyranoside }\end{array}$ & C. otophyllum & Roots & [16] \\
\hline 203 & $\begin{array}{l}\text { Qingyangshengenin 3-O- } \beta \text {-D-oleandropyranosyl- } \\
\qquad(1 \rightarrow 4)-\beta \text {-D-cymaropyranoside }\end{array}$ & C. otophyllum & Roots & [16] \\
\hline 204 & $\begin{array}{c}\text { Qingyangshengenin 3-O- } \beta \text {-D-oleandropyranosyl- } \\
(1 \rightarrow 4)-\beta \text {-D-cymaropyranosyl- }(1 \rightarrow 4)- \\
\beta \text {-D-digitoxopyranoside }\end{array}$ & C. otophyllum & Roots & [16] \\
\hline 205 & $\begin{array}{l}\text { Qingyangshengenin 3-O- } \beta \text {-D-cymaropyranosyl- } \\
(1 \rightarrow 4)-\beta \text {-D-thevetopyranosyl- }(1 \rightarrow 4)-\beta \text {-D- } \\
\text { cymaropyranosyl-( }(1 \rightarrow 4)-\beta \text {-D-digitoxopyranoside }\end{array}$ & C. otophyllum & Roots & [46] \\
\hline 206 & $\begin{array}{l}\text { Qingyangshengenin 3-O- } \alpha \text {-L-cymaropyranosyl- } \\
(1 \rightarrow 4)-\beta \text {-D-oleandropyranosyl- }(1 \rightarrow 4)-\beta \text {-D- } \\
\text { cymaropyranosyl-( }(1 \rightarrow 4) \text { - } \beta \text {-D-cymaropyranoside }\end{array}$ & C. otophyllum & Roots & [46] \\
\hline 207 & $\begin{array}{l}\text { Qingyangshengenin 3-O- } \alpha \text {-L-cymaropyranosyl- } \\
(1 \rightarrow 4)-\beta \text {-D-cymaropyranosyl- }(1 \rightarrow 4)-\beta \text {-D- } \\
\text { cymaropyranosyl- }(1 \rightarrow 4) \text { - } \alpha \text {-L-cymaropyranosyl- } \\
(1 \rightarrow 4)-\beta \text {-D-oleandropyranosyl- }(1 \rightarrow 4)-\beta \\
\text {-D-cymaropyranoside }\end{array}$ & C. otophyllum & Roots & [46] \\
\hline 208 & $\begin{array}{l}\text { Qinyangshengenin-3-O- } \beta \text {-D-oleandropyranosyl- } \\
(1 \rightarrow 4)-\beta \text {-D-oleandropyranosyl- }(1 \rightarrow 4)-\beta \text {-D- } \\
\text { cymaropyranosyl-( }(1 \rightarrow 4)-\beta \text {-D-cymaropyranoside }\end{array}$ & C. otophyllum & Roots & [52] \\
\hline
\end{tabular}


Table 3. Cont.

\begin{tabular}{|c|c|c|c|c|}
\hline No. & Compound Name & Species & Parts & Ref. \\
\hline 209 & $\begin{array}{l}\text { Qinyangshengenin-3-O- } \alpha \text {-L-cymaropyranosyl- } \\
(1 \rightarrow 4)-\beta \text {-D-oleandropyranosyl-( }(1 \rightarrow 4)-\beta \text {-D- } \\
\text { cymaropyranosyl- }(1 \rightarrow 4)-\beta \text {-D-digitoxopyranoside }\end{array}$ & C. wallichii & Roots & [53] \\
\hline 210 & Deacymetaplexigenin & C. wilfordii & Roots & [9] \\
\hline 211 & 12-O-vanilloyl-deacymetaplexigenin & C. wilfordii. & Roots & [9] \\
\hline 212 & 12-O-benzoyldeacymetaplexigenin & C. wilfordii. & Roots & [9] \\
\hline 213 & $\begin{array}{c}\text { 17 } \beta \text {-O-cinnamoyl-3 } \beta, 8 \beta, 14 \beta \text {-trihydroxypregn- } \\
12,20 \text {-ether }\end{array}$ & C. wilfordii. & Roots & [9] \\
\hline 214 & $\begin{array}{c}\text { Gagamine } 3-O-\beta \text {-D-oleandropyranosyl- } \\
(1 \rightarrow 4) \text { - } \beta \text {-D-cymaropyranosyl- }(1 \rightarrow 4)-\beta \text {-D- } \\
\text { cymaropyranoside }\end{array}$ & C. otophyllum & Roots & [46] \\
\hline 215 & $\begin{array}{c}\text { Gagaminin 3-O- } \beta \text {-D-cymaropyranosyl- } \\
(1 \rightarrow 4)-\beta \text {-D-oleandropyranosyl- }(1 \rightarrow 4)-\beta \text {-D- } \\
\text { cymaropyranosyl- }(1 \rightarrow 4)-\beta \text {-D-cymaropyranoside }\end{array}$ & C. wilfordii & Roots & [54] \\
\hline 216 & $\begin{array}{c}\text { Gagaminin 3-O- } \beta \text {-L-cymaropyranosyl- } \\
(1 \rightarrow 4)-\beta \text {-D-cymaropyranosyl- }(1 \rightarrow 4)-\alpha \text {-L- } \\
\text { diginopyranosyl- }(1 \rightarrow 4)-\beta \text {-D-digitoxopyranoside }\end{array}$ & C. bungei & Stems & [21] \\
\hline 217 & $\begin{array}{c}\text { Gagaminin 3-O- } \beta \text {-L-cymaropyranosyl- } \\
(1 \rightarrow 4)-\beta \text {-D-cymaropyranosyl- }(1 \rightarrow 4)-\alpha \text {-L- } \\
\text { diginopyranosyl- }(1 \rightarrow 4)-\beta \text {-D-cymaropyranoside }\end{array}$ & C. bungei & Stems & [21] \\
\hline 218 & $\begin{array}{l}\text { Gagaminine } 3-O-\beta \text {-D-oleandropyranosyl- } \\
(1 \rightarrow 4)-\beta \text {-D-oleandropyranosyl- }(1 \rightarrow 4)-\beta \text {-D- } \\
\text { cymaropyranoside }\end{array}$ & C. saccatum & Roots & [55] \\
\hline 219 & $\begin{array}{c}\text { Gagaminin 3-O- } \alpha \text {-L-cymaropyranosyl- } \\
(1 \rightarrow 4)-\beta \text {-D-cymaropyranosyl- }(1 \rightarrow 4)-\alpha \text {-L- } \\
\text { diginopyranosyl- }(1 \rightarrow 4)-\beta \text {-D-digitoxopyranoside }\end{array}$ & C. wilfordii. & Roots & [50] \\
\hline 220 & $\begin{array}{l}\text { Gagaminin-3-O- } \beta \text {-D-oleandropyranosyl- } \\
(1 \rightarrow 4)-\beta \text {-D-cymaropyranosyl- }(1 \rightarrow 4)-\beta \text {-D- } \\
\text { digitoxopyranoside }\end{array}$ & C. otophyllum & Roots & [46] \\
\hline 221 & $\begin{array}{l}12 \beta \text {-O-benzoyl-8 } \beta, 14 \beta, 17 \beta \text {-trihydroxypregn- } \\
2,5 \text {-diene-20-one }\end{array}$ & C. wilfordii. & Roots & [9] \\
\hline 222 & Rostratamin & C. wilfordii. & Roots & [9] \\
\hline 223 & $\begin{array}{l}\text { Rostratamine } 3-O-\beta \text {-D-oleandropyranosyl- } \\
(1 \rightarrow 4) \text { - } \beta \text {-D-cymaropyranosyl- }(1 \rightarrow 4)-\beta \text {-D- } \\
\text { cymaropyranoside }\end{array}$ & C. otophyllum & Roots & [16] \\
\hline 224 & Sarcostin & C. otophyllum & Roots & [47] \\
\hline 225 & $\begin{array}{l}\text { 12-O-nicotinoylsarcostin3-O- } \beta \text {-L-cymaropyranosyl- } \\
(1 \rightarrow 4)-\beta \text {-D-cymaropyranosyl-( } 1 \rightarrow 4)-\alpha \text {-L- } \\
\text { diginopyranosyl- }(1 \rightarrow 4)-\beta \text {-D-cymaropyranoside }\end{array}$ & C. bungei & Stems & [21] \\
\hline 226 & $\begin{array}{l}\text { 12-O-acetylsarcostin 3-O- } \beta \text {-lcymaropyranosyl- } \\
(1 \rightarrow 4)-\beta \text {-D-cymaropyranosyl- }(1 \rightarrow 4)-\beta \text {-L- } \\
\text { cymaropyranosyl- }(1 \rightarrow 4)-\beta \text {-D-digitoxopyranosyl- } \\
(1 \rightarrow 4)-\beta \text {-D-digitoxopyranoside }\end{array}$ & C. bungei & Stems & [21] \\
\hline 227 & $\begin{array}{c}\text { 12-O-acetylsarcostin3-O- } \beta \text {-L-cymaropyranosyl- } \\
(1 \rightarrow 4)-\beta \text {-D-digitoxopyranosyl- }(1 \rightarrow 4)-\beta \text {-L- } \\
\text { cymaropyranosyl- }(1 \rightarrow 4) \text { - } \beta \text {-D-cymaropyranosyl- } \\
(1 \rightarrow 4)-\alpha \text {-L-diginopyranosyl- }(1 \rightarrow 4) \text { - } \\
\beta \text {-D-cymaropyranoside }\end{array}$ & C. bungei & Stems & [21] \\
\hline
\end{tabular}


Table 3. Cont.

\begin{tabular}{|c|c|c|c|c|}
\hline No. & Compound Name & Species & Parts & Ref. \\
\hline 228 & $\begin{array}{c}\text { 20-O-acetyl-12-O-cinnamoyl-3-O-( } \beta \text {-D- } \\
\text { oleandropyranosyl- }(1 \rightarrow 4)-\beta \text {-D-oleandropyranosyl- } \\
(1 \rightarrow 4)-\beta \text {-D-cymaropyranosyl)-8,14- } \\
\text { secosarcostin- } 8,14 \text {-dione }\end{array}$ & C. saccatum & Roots & [56] \\
\hline 229 & Deacylcynanchogenin & C. wilfordii & Roots & [9] \\
\hline 230 & Cynauricuoside A & C. wilfordii & Roots & [27] \\
\hline 231 & Cynauricuoside C & C. auriculatum & Root & [57] \\
\hline 232 & Cynanside A & C. aniculatum & Roots & [58] \\
\hline 233 & Cynanside B & C. aniculatum & Roots & [58] \\
\hline 234 & Komaroside C & C. forrestii & Roots & [59] \\
\hline 235 & Komaroside D & C. komarovii & Roots & [33] \\
\hline 236 & Komaroside E & C. komarovii & Roots & [33] \\
\hline 237 & Komaroside F & C. komarovii & Roots & [33] \\
\hline 238 & Komaroside G & C. komarovii & Roots & [33] \\
\hline 239 & Komaroside $\mathrm{H}$ & C. komarovii & Roots & [33] \\
\hline 240 & Cynauricoside A & C. wilfordii. & Roots & [50] \\
\hline 241 & Cynauricoside B & C. auriculatum & Roots & [30] \\
\hline 242 & Cynauricoside C & C. auriculatum & Roots & [30] \\
\hline 243 & Cynauricoside D & C. auriculatum & Roots & [30] \\
\hline 244 & Cynauricoside $\mathrm{E}$ & C. auriculatum & Roots & [30] \\
\hline 245 & Cynauricoside $\mathrm{F}$ & C. auriculatum & Roots & [30] \\
\hline 246 & Cynauricoside G & C. auriculatum & Roots & [30] \\
\hline 247 & Cynauricoside $\mathrm{H}$ & C. auriculatum & Roots & [30] \\
\hline 248 & Cynauricoside I & C. auriculatum & Roots & [30] \\
\hline 249 & Cynauricuside A & C. auriculatum & Roots & [30] \\
\hline 250 & Cynaforroside B & C. forrestii & Roots & [59] \\
\hline 251 & Cynaforroside $\mathrm{C}$ & C. forrestii & Roots & [59] \\
\hline 252 & Cynaforroside D & C. forrestii & Roots & [59] \\
\hline 253 & Cynaforroside E & C. forrestii & Roots & [59] \\
\hline 254 & Cynaforroside F & C. forrestii & Roots & [59] \\
\hline 255 & Cynaforroside G & C. forrestii & Roots & [59] \\
\hline 256 & Cynaforroside $\mathrm{H}$ & C. forrestii & Roots & [59] \\
\hline 257 & Cynaforroside I & C. forrestii & Roots & [59] \\
\hline 258 & Cynaforroside J & C. forrestii & Roots & [59] \\
\hline 259 & Cynaforroside $\mathrm{K}$ & C. forrestii & Roots & [60] \\
\hline 260 & Cynaforroside L & C. forrestii & Roots & [60] \\
\hline 261 & Cynaforroside M & C. forrestii & Roots & [60] \\
\hline 262 & Cynaforroside $\mathrm{N}$ & C. forrestii & Roots & [60] \\
\hline 263 & Cynaforroside $\mathrm{O}$ & C. forrestii & Roots & [60] \\
\hline 264 & Cynaforroside P & C. forrestii & Roots & [60] \\
\hline 265 & Cynaforroside $Q$ & C. forrestii & Roots & [60] \\
\hline 266 & Atratoglaucoside A & $\begin{array}{l}\text { C. atratum } \\
\text { C. versicolor }\end{array}$ & Roots & [39] \\
\hline
\end{tabular}


Table 3. Cont.

\begin{tabular}{|c|c|c|c|c|}
\hline No. & Compound Name & Species & Parts & Ref. \\
\hline 267 & Atratoglaucoside B & C. atratum & Roots & [39] \\
\hline 268 & Paniculatumoside A & C. paniculatum. & Roots & [61] \\
\hline 269 & Paniculatumoside B & C. paniculatum. & Roots & [61] \\
\hline 270 & Neohancoside C & C. hunmkiunum & Roots & [62] \\
\hline 271 & Neohancoside D & C. hunmkiunum & Roots & [62] \\
\hline 272 & $\begin{array}{c}\text { Deoxyamplexicogenin A-3-O-yl-4-O-(4-O- } \\
\alpha \text {-L-cymaropyranosoyl- } \beta \text {-D-digitoxopyranosoyl)- } \\
\beta \text {-D-canaropyranoside }\end{array}$ & C. stauntonii & Roots & [63] \\
\hline 273 & 2-deoxyamplexicogenin A & C. stauntonii & Roots & [64] \\
\hline 274 & Amplexicogenin C-3-O- $\beta$-D-cymaropyranoside & C. amplexicaule & Roots & [65] \\
\hline 275 & Cynascyroside A & C. ascyrifolium & Roots & [66] \\
\hline 276 & Cynascyroside B & C. ascyrifolium & Roots & [66] \\
\hline 277 & Cynascyroside C & $\begin{array}{l}\text { C. ascyrifolium } \\
\text { C. chekiangense }\end{array}$ & Roots & [66] \\
\hline 278 & Cynascyroside D & C. atratum & Roots & [25] \\
\hline 279 & Taiwanoside A & C. taiwanianum & Roots & [28] \\
\hline 280 & Taiwanoside B & C. taiwanianum & Roots & [28] \\
\hline 281 & Taiwanoside C & C. taiwanianum & Roots & [28] \\
\hline 282 & Taiwanoside D & C. taiwanianum & Roots & [28] \\
\hline 283 & Taiwanoside E & C. taiwanianum & Roots & [28] \\
\hline 284 & Stauntonine & C. stauntonii & Roots & [40] \\
\hline 285 & Anhydrohirundigenin & C. stauntonii & Roots & [40] \\
\hline 286 & Anhydrohirundigenin monothevetoside & C. stauntonii & Roots & [40] \\
\hline 287 & Auriculoside I & C. auriculatum & Roots & [29] \\
\hline 288 & Auriculoside II & C. auriculatum & Roots & [29] \\
\hline 289 & Auriculoside III & C. auriculatum & Roots & [29] \\
\hline 290 & Auriculoside IV & C. auriculatum & Roots & [29] \\
\hline 291 & Cynanauriculoside I & C. auriculatum & Roots & [29] \\
\hline 292 & Cynanauriculoside II & C. auriculatum & Roots & [29] \\
\hline 293 & Cynanauriculoside A & C. wallichii & Roots & [53] \\
\hline 294 & Cynanauriculoside $\mathrm{C}$ & C. auriculatum & Roots & [67] \\
\hline 295 & Cynanauriculoside D & C. auriculatum & Roots & [67] \\
\hline 296 & Cynanauriculoside $\mathrm{E}$ & C. auriculatum & Roots & [67] \\
\hline 297 & 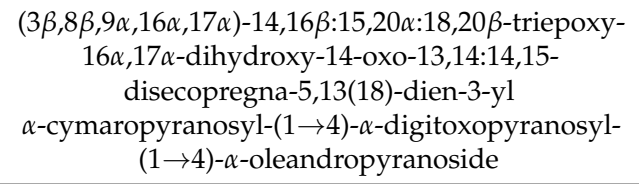 & C. paniculatum & Stems & [68] \\
\hline 298 & 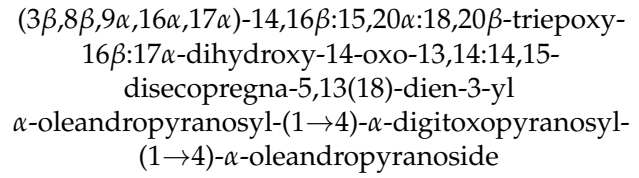 & C. paniculatum & Stems & [68] \\
\hline
\end{tabular}


Table 3. Cont.

\begin{tabular}{|c|c|c|c|c|}
\hline No. & Compound Name & Species & Parts & Ref. \\
\hline 299 & Cyanoauriculoside $\mathrm{C}$ & C. auriculatum & Roots & [69] \\
\hline 300 & Cyanoauriculoside D & C. auriculatum & Roots & [69] \\
\hline 301 & Cyanoauriculoside E & C. auriculatum & Roots & [69] \\
\hline 302 & Cyanoauriculoside G & C. wilfordii. & Roots & [50] \\
\hline 303 & Hirundoside A & C. stauntonii & Roots & [43] \\
\hline 304 & Deacetylmetaplexigenin & C. otophyllum & Roots & [47] \\
\hline 305 & $\begin{array}{l}\text { Deacetylmetaplexigenin 3-O- } \beta \text {-D- } \\
\text { oleandropyranosyl- }(1 \rightarrow 4)-\alpha \text {-D-oleandropyranosyl- } \\
(1 \rightarrow 4)-\alpha \text {-D-oleandropyranoside }\end{array}$ & C. otophyllum & Rhizome & [70] \\
\hline 306 & $\begin{array}{l}\text { Deacetylmetaplexigenin 3-O- } \alpha \text {-D- } \\
\text { oleandropyranosyl- }(1 \rightarrow 4)-\beta \text {-D-thevetopyranosyl- } \\
(1 \rightarrow 4)-\alpha \text {-D-oleandropyranoside }\end{array}$ & C. otophyllum & Rhizome & [70] \\
\hline 307 & $\begin{array}{l}\text { Deacetylmetaplexigenin 3-O- } \beta \text {-D- } \\
\text { cymaropyranosyl- }(1 \rightarrow 4)-\alpha \text {-D-oleandropyranoside }\end{array}$ & C. otophyllum & Rhizome & [70] \\
\hline 308 & Cynsaccatol A & C. saccatum & Roots & [55] \\
\hline 309 & Cynsaccatol B & C. saccatum & Roots & [55] \\
\hline 310 & Cynsaccatol C & C. saccatum & Roots & [55] \\
\hline 311 & Cynsaccatol D & C. saccatum & Roots & [55] \\
\hline 312 & Cynsaccatol E & C. saccatum & Roots & [55] \\
\hline 313 & Cynsaccatol F & C. saccatum & Roots & [55] \\
\hline 314 & Cynsaccatol G & C. saccatum & Roots & [55] \\
\hline 315 & Cynsaccatol H & C. saccatum & Roots & [55] \\
\hline 316 & Cynotophylloside A & C. otophyllum. & Roots & [47] \\
\hline 317 & Cynotophylloside B & C. otophyllum. & Roots & [47] \\
\hline 318 & Cynotophylloside C & C. otophyllum. & Roots & [47] \\
\hline 319 & Cynotophylloside D & C. otophyllum. & Roots & [47] \\
\hline 320 & Cynotophylloside E & C. otophyllum. & Roots & [47] \\
\hline 321 & Cynotophylloside F & C. otophyllum. & Roots & [47] \\
\hline 322 & Cynotophylloside H & C.otophyllum & Roots/stems & [71] \\
\hline 323 & Stephanoside H & C. otophyllum & Roots & [46] \\
\hline 324 & Wallicoside & C. otophyllum & Roots & [18] \\
\hline 325 & Wallicoside J & C. otophyllum & Roots & [46] \\
\hline 326 & Cynawilfoside A & C. wilfordii. & Roots & [50] \\
\hline 327 & Cynawilfoside B & C. wilfordii. & Roots & [50] \\
\hline 328 & Cynawilfoside C & C. wilfordii. & Roots & [50] \\
\hline 329 & Cynawilfoside D & C. wilfordii. & Roots & [50] \\
\hline 330 & Cynawilfoside E & C. wilfordii. & Roots & [50] \\
\hline 331 & Cynawilfoside F & C. wilfordii. & Roots & [50] \\
\hline 332 & Cynawilfoside G & C. wilfordii. & Roots & [50] \\
\hline 333 & Cynawilfoside $\mathrm{H}$ & C. wilfordii. & Roots & [50] \\
\hline 334 & Cynawilfoside I & C. wilfordii. & Roots & [50] \\
\hline 335 & Atratcynoside A & C. atratum & Roots & [72] \\
\hline 336 & Atratcynoside B & C. atratum & Roots & [72] \\
\hline 337 & Atratcynoside C & C. atratum & Roots & [72] \\
\hline 338 & Atratcynoside D & C. atratum & Roots & [72] \\
\hline
\end{tabular}


Table 3. Cont.

\begin{tabular}{|c|c|c|c|c|}
\hline No. & Compound Name & Species & Parts & Ref. \\
\hline 339 & Atratcynoside E & C. atratum & Roots & [72] \\
\hline 340 & Atratcynoside F & C. atratum & Roots & [72] \\
\hline 341 & Mooreanoside A & C. mooreanum & Roots & [73] \\
\hline 342 & Mooreanoside B & C. mooreanum & Roots & [73] \\
\hline 343 & Mooreanoside C & C. mooreanum & Roots & [73] \\
\hline 344 & Mooreanoside D & C. mooreanum & Roots & [73] \\
\hline 345 & Mooreanoside E & C. mooreanum & Roots & [73] \\
\hline 346 & Mooreanoside F & C. mooreanum & Roots & [73] \\
\hline 347 & Mooreanoside G & C. mooreanum & Roots & [73] \\
\hline 348 & Mooreanoside $\mathrm{H}$ & C. mooreanum & Roots & [73] \\
\hline 349 & Mooreanoside I & C. mooreanum & Roots & [73] \\
\hline 350 & Mooreanoside J & C. mooreanum & Roots & [73] \\
\hline 351 & Mooreanoside K & C. mooreanum & Roots & [73] \\
\hline 352 & Mooreanoside L & C. mooreanum & Roots & [73] \\
\hline 353 & Mooreanoside M & C. mooreanum & Roots & [73] \\
\hline 354 & Mooreanoside N & C. mooreanum & Roots & [73] \\
\hline 355 & Mooreanoside $\mathrm{O}$ & C. mooreanum & Roots & [73] \\
\hline 356 & Mooreanoside P & C. mooreanum & Roots & [73] \\
\hline 357 & Cynastauoside A & C. stauntonii & Roots & [74] \\
\hline 358 & Cynastauoside B & C. stauntonii & Roots & [74] \\
\hline 359 & Cynastauoside $\mathrm{C}$ & C. stauntonii & Roots & [74] \\
\hline 360 & Saccatol A & C. saccatum & Roots & [56] \\
\hline 361 & Saccatol B & C. saccatum & Roots & [56] \\
\hline 362 & Saccatol C & C. saccatum & Roots & [56] \\
\hline 363 & Cynanotoside A & C. otophyllum & Roots/stems & [71] \\
\hline 364 & Cynanotoside B & C. otophyllum & Roots/stems & [71] \\
\hline 365 & Cynanotoside $\mathrm{C}$ & C. otophyllum & Roots/stems & [71] \\
\hline 366 & Cynanotoside D & C. otophyllum & Roots/stems & [71] \\
\hline 367 & Cynanotoside E & C. otophyllum & Roots/stems & [71] \\
\hline 368 & Mucronatoside C & C. otophyllum & Roots & [46] \\
\hline 369 & Sinomarinoside B & C. otophyllum & Roots & [46] \\
\hline 370 & Cynanotophylloside A & C. otophyllum & Roots & [31] \\
\hline 371 & Cynanotophylloside B & C. otophyllum & Roots & [31] \\
\hline 372 & Cynanotophylloside C & C. otophyllum & Roots & [31] \\
\hline 373 & Cynanotophylloside D & C. otophyllum & Roots & [31] \\
\hline 374 & Cynanauriculatoside A & C. otophyllum & Roots & [31] \\
\hline 375 & $3 \beta, 14 \beta$-dihydroxy-14 $\beta$-pregn-5-en-20-one & C. paniculatum. & Root/rhizome & [41] \\
\hline 376 & 3-O- $\beta$-D-oleandropanyanoside & C. paniculatum. & Root/rhizome & [41] \\
\hline 377 & Hancopregnane & C. hunmkiunum & Roots & [37] \\
\hline 378 & Menarandroside A & $\begin{array}{c}\text { C. } \\
\text { menarandrense }\end{array}$ & Aerial parts & [75] \\
\hline
\end{tabular}


Table 3. Cont.

\begin{tabular}{|c|c|c|c|c|}
\hline No. & Compound Name & Species & Parts & Ref. \\
\hline 379 & Menarandroside B & $\begin{array}{c}\mathrm{C} . \\
\text { menarandrense }\end{array}$ & Aerial parts & [75] \\
\hline 380 & Menarandroside C & $\begin{array}{c}\text { C. } \\
\text { menarandrense }\end{array}$ & Aerial parts & [75] \\
\hline 381 & Menarandroside D & $\begin{array}{c}\text { C. } \\
\text { menarandrense }\end{array}$ & Aerial parts & [75] \\
\hline 382 & Menarandroside E & $\begin{array}{c}\mathrm{C} . \\
\text { menarandrense }\end{array}$ & Aerial parts & [75] \\
\hline 383 & Carumbelloside I & $\begin{array}{c}\text { C. } \\
\text { menarandrense }\end{array}$ & Aerial parts & [75] \\
\hline 384 & Carumbelloside II & $\begin{array}{c}\text { C. } \\
\text { menarandrense }\end{array}$ & Aerial parts & [75] \\
\hline 385 & Pregnenolone-3-O-gentiobioside & $\begin{array}{c}\text { C. } \\
\text { menarandrense }\end{array}$ & Aerial parts & [75] \\
\hline 386 & 14-O-methyl-3-epi-hirundigenin & C. stauntonii & Roots & [76] \\
\hline 387 & Stauntosaponin A & C. stauntonii & Roots & [77] \\
\hline 388 & Stauntosaponin B & C. stauntonii & Roots & [77] \\
\hline \multicolumn{5}{|c|}{ Benzene and its derivatives } \\
\hline 389 & Cynantetrone & C. taiwanianum & Rhizome & [78] \\
\hline 390 & CynantetroneA & C. taiwanianum & Rhizome & [78] \\
\hline 391 & Cynandione A & C. taiwanianum & Rhizome & [78] \\
\hline 392 & Cynandione B & C. taiwanianum & Rhizome & [78] \\
\hline 393 & 2,4-Dihydroxyacetophenone & C. atratum & Roots & [25] \\
\hline 394 & 2,5-Dihydroxyacetophenone & C. bungei & Roots & [79] \\
\hline 395 & 4-Hydroxyacetophenone & C. atratum & Roots & [25] \\
\hline 396 & 4-acetylphenol & C. paniculatum & Roots & [80] \\
\hline 397 & 2,5-dihydroxy-4-methoxyacetophenone & C. paniculatum & Roots & [80] \\
\hline 398 & 2,3-dihydroxy-4-methoxyacetophenone & C. paniculatum & Roots & [81] \\
\hline 399 & Acetoveratrone & C. paniculatum & Roots & [80] \\
\hline 400 & 2,5-dimethoxyhydroquinone & C. paniculatum & Roots & [80] \\
\hline 401 & Resacetophenone & C. paniculatum & Roots & [80] \\
\hline 402 & M-acetylphenol & C. paniculatum & Roots & [80] \\
\hline 403 & Vanillic acid & C. paniculatum & Roots & [80] \\
\hline 404 & 3,5-dimethoxyhydroquinone & C. paniculatum & Roots & [80] \\
\hline 405 & Acetovanillone & C. wilfordii & Roots & [3] \\
\hline 406 & p-hydroxyacetophenone & C. wilfordii & Roots & [3] \\
\hline 407 & $\begin{array}{l}\text { 3-( } \beta \text {-D-ribofuranosyl)-2,3-dihydro-6H- } \\
\text { 1,3-oxazine-2,6-dione }\end{array}$ & C. wilfordii & Roots & [3] \\
\hline 408 & Bungeiside A & C. wilfordii & Roots & [3] \\
\hline 409 & Cynanoneside B & C. wilfordii & Roots & [3] \\
\hline 410 & Cynanoneside A & C. taiwanianum & Roots & [82] \\
\hline 411 & Baishouwubenzophenone & C. auriculatum & Roots & [83] \\
\hline 412 & 3,4-dihydroxyacetophenone & C. atratum & Roots & [39] \\
\hline
\end{tabular}


Table 3. Cont.

\begin{tabular}{|c|c|c|c|c|}
\hline No. & Compound Name & Species & Parts & Ref. \\
\hline 413 & $4^{\prime}$-hydroxy-3'-methoxyacetophenone & C. wilfordii & Roots & [84] \\
\hline 414 & Paeonol & C. auriculatum & Roots & [58] \\
\hline 415 & Isopaeonol & C. auriculatum & Roots & [58] \\
\hline 416 & 2-hydroxy-5-methoxyacetophenone & C. auriculatum & Roots & [58] \\
\hline 417 & Caffeic acid & C. taiwanianum & Aerial parts & [85] \\
\hline 418 & Syringic acid & C. paniculatum & Roots & {$[86]$} \\
\hline \multicolumn{5}{|c|}{ Alkaloids } \\
\hline 419 & Gagamine & C. caudatum & Roots & [87] \\
\hline 420 & Antofine & C. vincetoxicum & Aerial parts & [88] \\
\hline 421 & Tylophorine & C. vincetoxicum & Aerial parts & [88] \\
\hline 422 & Vincetene & C. vincetoxicum & Aerial parts & {$[88,89]$} \\
\hline 423 & (-)-10 $\beta, 13 \mathrm{a} \alpha-14 \beta$-hydroxyantofine $N$-oxide & C. vincetoxicum & Aerial parts & [90] \\
\hline 424 & $(-)-10 \beta, 13 \mathrm{a} \alpha$-secoantofine $N$-oxide & C. vincetoxicum & Aerial parts & [90] \\
\hline 425 & $(-)-(R)-13 \mathrm{a} \alpha-6-O-$ desmethylantofine & C. vincetoxicum & Aerial parts & [91] \\
\hline 426 & $(-)-(R)-13 \mathrm{a} \alpha$-secoantofine & C. vincetoxicum & Aerial parts & [91] \\
\hline 427 & $(-)-(R)-13 \mathrm{a} \alpha-6-O-$ desmethylsecoantofine & C. vincetoxicum & Aerial parts & [91] \\
\hline 428 & (-)-10 $\beta$-antofine $N$-oxide & C. vincetoxicum & Aerial parts & [90] \\
\hline 429 & $\begin{array}{l}\text { 2,3-dimethoxy-6-(3-oxo-butyl)-7,9,10,11,11a, } \\
\text { 12-hexahydrobenzo[f]pyrrolo[1,2-b]isoquinoline }\end{array}$ & C. komarovii & Aerial parts & [92] \\
\hline 430 & 7-demethoxytylophorine & C. komarovii & Aerial parts & [92] \\
\hline 431 & 7-demethoxytylophorine $N$-oxide & C. komarovii & Aerial parts & [92] \\
\hline \multicolumn{5}{|c|}{ Flavones } \\
\hline 432 & $\begin{array}{l}\text { 7-O- } \alpha \text {-L-rhamnopyranosyl-kaempferol-3- } \\
\text { O- } \beta \text {-D-glucopyranoside }\end{array}$ & C. chinese & Aerial parts & [93] \\
\hline 433 & $\begin{array}{l}\text { 7-O- } \alpha \text {-L-rhamnopyranosyl-kaempferol-3- } \\
\text { O- } \alpha \text {-L-rhamnopyranoside }\end{array}$ & C. chinese & Aerial parts & [93] \\
\hline 434 & Kaempferol & C. taizwanianum & Aerial parts & [85] \\
\hline 435 & Astragalin & C. taiwanianum & Aerial parts & [85] \\
\hline 436 & Afzelin & C. taiwanianum & Aerial parts & [85] \\
\hline 437 & Trifolin & C. taiwanianum & Aerial parts & [85] \\
\hline 438 & Quercetin & C. taizwanianum & Aerial parts & [85] \\
\hline 439 & Isoquercitrin & C. taiwanianum & Aerial parts & [85] \\
\hline 440 & Quercitrin & C. taiwanianum & Aerial parts & [85] \\
\hline 441 & Hyperin & C. taizwanianum & Aerial parts & [85] \\
\hline \multicolumn{5}{|c|}{ Terpene } \\
\hline 442 & Neohancoside A & C. hunmkiunum & Roots & {$[34]$} \\
\hline 443 & Neohancoside B & C. hunmkiunum & Roots & [62] \\
\hline 444 & $\beta$-amyrin & C. paniculatum & Roots & [86] \\
\hline 445 & $\alpha$-amyrin & C. paniculatum & Roots & [86] \\
\hline 446 & Lupeol & C. paniculatum & Roots & [86] \\
\hline 447 & Taraxasterol & C. paniculatum & Roots & [86] \\
\hline 448 & Ursolic acid & C. paniculatum & Roots & [86] \\
\hline 449 & Oleanolic acid & C. paniculatum & Roots & [86] \\
\hline 450 & Maslinic acid & C. paniculatum & Roots & [86] \\
\hline
\end{tabular}




\subsection{C21 Steroids}

The C21 steroid compounds all have the basic skeleton of pregnane, which containing 21 carbon atoms or a derivative of its isomers. C21 steroid constituents in Cynanchum sp. can be classified into two groups on the basis of their carbon frameworks as typical and modified C21 steroids. According to the different pregnane skeletons, these compounds can be finally divided into the following five types: the normal four-ring pregnane type, 14,15-secopregnanetype, 13,14:14,15-diseco-pregnane type, aberrant 14,15-seco-pregnane type and 12,13-seco-14,18-nor-pregnane type. In C21 steroidal glycosides, sugar moiety is linked most frequently at C-3 to a hydroxyl group of the pregnane aglycone, which contains one to seven sugar units with mode of $1 \rightarrow 4$, and is generally composed of a linear (rather than a branched) oligosaccharide chain. The most common sugar residues are hexose (glucose), 6-deoxyhexose (thevetose) and 2,6-dideoxyhexoses (cymarose, oleandrose, digitoxose, diginose, sarmentose and canarose). In 2016, Gu et al. on the C21 steroid have been comprehensively and fully explained [2]. Therefore, we summarized the newly isolated compounds from Cynanchum sp. in 2016-2017 (Figure 1).

\subsection{Benzene and Its Derivatives}

Benzene and its derivatives are also found in Cynanchum plants. These components are mainly acetophenone derivatives, and most of them were isolated from C. paniculatum, C. auriculatum and C. stauntonii. The acetophenones in Cynanchum sp. include cynantetrone (389), cynantetrone A (390), cynandione A (391), cynandione B (392) [78], 2,4-dihydroxyacetophenone (393), 2,5-dihydroxyacetophenone (394) [79], 4-hydroxyacetophenone (395) [25], 4-acetylphenol (396), 2,5-dihydroxy-4-methoxyacetophenone (397), 2,3-dihydroxy-4-methoxyacetophenone (398) [81], acetoveratrone (399), 2,5-dimethoxyhydroquinone (400), resacetophenone (401), $m$-acetylphenol (402), vanillic acid (403), 3,5-dimethoxyhydroquinone (404) [80], acetovanillone (405), $p$-hydroxyacetophenone (406), 3-( $\beta$-D-ribofuranosyl)-2,3-dihydro-6H-1,3-oxazine-2,6-dione (407), bungeiside A (408), cynanoneside B (409) [3], cynanoneside A (410) [82], baishouwubenzophenone (411) [83], 3,4-dihydroxyacetophenone (412) [39], 4'-hydroxy-3'-methoxyacetophenone (413) [84], paeonol (414), isopaeonol (415), 2-hydroxy-5-methoxyacetophenone (416) [86], caffeic acid (417) [85] and syringic acid (418) [25]. Structures of these compounds are shown in Figure 2.

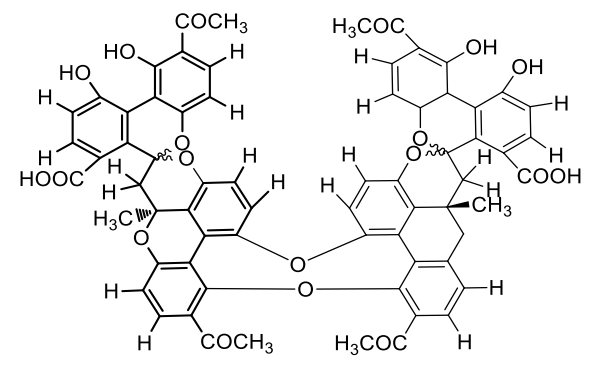

(389)

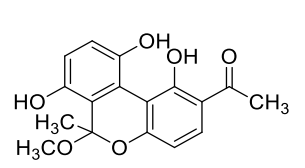

(391)

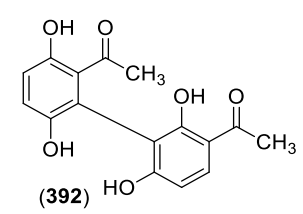

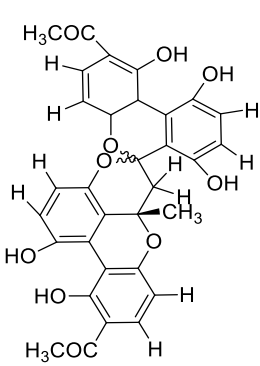

(390)

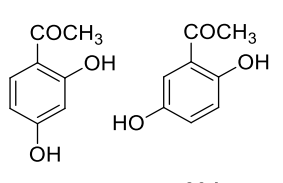

(393) (394)<smiles>COc1cc(O)c(C(C)=O)cc1O</smiles><smiles>COc1ccc(C(C)=O)cc1OC</smiles><smiles>COc1cc(O)c(OC)cc1O</smiles>

(400)

Figure 2. Cont. 
<smiles>COc1cc(C(=O)O)ccc1OC(C)(C)C(=O)Oc1cccc(C(=O)c2ccc(O)cc2C=O)c1</smiles><smiles>CC(=O)c1ccc(O)cc1</smiles><smiles>O=C1C=CN2C(O1)[C@@H](O)[C@H](O)[C@H](O)[C@H]2O</smiles><smiles>[R19]CC(=O)c1cc([R])c([R])cc1Br</smiles><smiles>[R]Oc1ccc(C(C)=O)cc1O</smiles>

(406)

(407)<smiles>CC(=O)c1ccc(O)c(C(=O)c2c(O)ccc(O)c2C)c1O</smiles><smiles>CC(=O)c1ccc(O)c(O)c1</smiles>

(412)<smiles>COc1ccc(O)c(C(C)=O)c1</smiles>

(413)<smiles>COc1ccc(C(C)=O)c(O)c1</smiles>

(414)<smiles>COc1ccc(C(C)=O)cc1O</smiles>

(415)<smiles>COc1ccc(O)c(C(C)=O)c1</smiles>

(416)<smiles>O=C(O)C=Cc1ccc(O)c(O)c1</smiles>

(417)<smiles>COc1cc(C(=O)O)cc(OC)c1O</smiles>

(418)

Figure 2. Structures of compounds 389-418 from Cynanchum species.

\subsection{Alkaloids}

Studies showed that alkaloids are only found in several plants of genus Cynanchum, and some of these alkaloids showed notable bioactivity. To date, 13 alkaloids were identified from genus Cynanchum. These alkaloids include a steroidal alkaloid gagaminine (419) [94] and fourteen phenanthroindolizidine alkaloids. The phenanthroindolizidine is an alkaloid with a basic skeleton that is a pentacyclic structure with a phenanthrene ring and a indolizidine ring, in which the phenanthrene ring contains a plurality of methoxy groups or hydroxyl groups, and some of the alkaloids also contain a methyl group or a hydroxyl group on the indolizidine ring. In this type of alkaloid, the phenanthrene ring of some compounds is not formed, and some compounds are nitrogen oxides. In addition to compound 419, compounds $\mathbf{4 2 0 - 4 3 2}$ have been identified as phenanthroindolizidine alkaloids. These compounds were isolated from aerial parts of $C$. vincetoxicum and identified as antofine (420), tylophorine (421), vincetene (422) [88], (-)-10 $\beta, 13 \mathrm{a} \alpha-14 \beta$-hydroxyantofine $N$-oxide (423), (-)-10- $\beta, 13 \mathrm{a} \alpha$-secoantofine $N$-oxide (424) [90], (-)-(R)-13a $\alpha$-6-O-desmethylantofine (425), (-)-(R)-13a $\alpha$-secoantofine (426), (-)-(R)-13a $\alpha-6-O-$-desmethylsecoantofine (427) [91], (-)-10 $\beta$-antofine $N$-oxide (428) [90], 2,3-dimethoxy-6-(3-oxo-butyl)-7,9,10,11,11a,12-hexahydrobenzo[f]pyrrolo[1,2-b]isoquinoline (429), 7-demethoxytylophorine (430) and 7-demethoxy-tylophorine $N$-oxide (431) [92]. Structures of these compounds are shown in Figure 3. 
<smiles>CCOC(=O)c1cccnc1</smiles>

(419)<smiles>COc1ccc2c3c(c4cc(OC)c(OC)cc4c2c1)CC1CCCN1C3</smiles>

(420)<smiles>COc1ccc(C2=C(c3ccc(OC)c(OC)c3)C[C@@H]3CCC[N+]3([O-])C2)cc1</smiles>

(424)<smiles>COc1ccc2c3c(c4cc(OC)c(OC)cc4c2c1)C[N+]1([O-])CCC[C@H]1C3</smiles>

(428)<smiles>COc1cc2c3c(c4cc(OC)c(OC)cc4c2cc1OC)CN1CCCC1C3</smiles>

(421)<smiles>[R]c1cc2c3c(c4cc([R])c([R])cc4c2cc1[R])C([R8])C1CCCN1C3</smiles><smiles>COc1cc2cc(/C=C\C(C)O)c3c(c2cc1OC)CC1CCCN1C3</smiles>

(422)<smiles>COc1ccc(C2=C(c3ccc(OC)c(OC)c3)CN3CCC[C@H]3C2)cc1</smiles>

(426)<smiles>COc1ccc2c3c(c4ccc(OC)c(OC)c4c2c1)C[C@@H]1CCCN1C3</smiles>

(430)<smiles>COc1ccc2c3c(c4cc(OC)c(OC)cc4c2c1)C(O)[C@H]1CCC[N+]1([O-])C3</smiles>

(423)<smiles>COc1ccc(C2=C(c3ccc(O)cc3)CN3CCC[C@H]3C2)cc1OC</smiles>

(427)<smiles>COc1ccc2c3c(c4ccc(OC)c(OC)c4c2c1)C[N+]1([O-])CCC[C@H]1C3</smiles>

(431)

Figure 3. Structures of compounds 419-431 from Cynanchum species.

\subsection{Flavones}

To date, there are few flavonoids isolated and identified from genus Cynanchum and most of them are flavonoid glycosides with 3- or 7-linked glycans. 7-O- $\alpha$-L-rhamnopyranosyl-kaempferol-3-O- $\beta$-Dglucopyranoside (432) and 7-O- $\alpha$-L-rhamnopyranosyl-kaempferol-3-O- $\alpha$-L-rhamnopyranoside (433) were identified from C. chinense [93]. Eight flavone components kaempferol (434), astragalin (435), afzelin (436), trifolin (437), quercetin (438), isoquercitrin (439), quercitrin (440) and hyperin (441) [85] were isolated from the aerial part of $C$. taiwanianum. Structures of these compounds are shown in Figure 4. 
<smiles>[R20]Oc1cc(O)c2c(=O)c(O[R2])c(-c3ccc(O)c([R7])c3)oc2c1</smiles>

$\begin{array}{lllc} & \mathrm{R}_{1} & \mathrm{R}_{2} & \mathrm{R}_{3} \\ \mathbf{( 4 3 2 )} & \mathrm{H} & \text { glucosyl } & \text { rhamnosyl } \\ \mathbf{( 4 3 3 )} & \mathrm{H} & \text { rhamnosyl } & \text { rhamnosyl } \\ \mathbf{( 4 3 4 )} & \mathrm{H} & \mathrm{H} & \mathrm{H} \\ \mathbf{( 4 3 5 )} & \mathrm{H} & \text { glucosyl } & \mathrm{H} \\ \mathbf{( 4 3 6 )} & \mathrm{H} & \text { rhamnosyl } & \mathrm{H} \\ \mathbf{( 4 3 7 )} & \mathrm{H} & \text { galactosyl } & \mathrm{H} \\ \mathbf{( 4 3 8 )} & \mathrm{OH} & \mathrm{H} & \mathrm{H} \\ \mathbf{( 4 3 9 )} & \mathrm{OH} & \text { glucosyl } & \mathrm{H} \\ \mathbf{( 4 4 0 )} & \mathrm{OH} & \text { rhamnosyl } & \mathrm{H} \\ (\mathbf{4 4 1}) & \mathrm{OH} & \text { galaclosyl } & \mathrm{H}\end{array}$<smiles>CC1OC2C(O)C(Oc3cc(O)c4c(=O)c(OC5OC(O)C(O)C(O)C(O)C5O)c(-c5ccc(O)cc5)oc4c3)OC12</smiles>

(432)

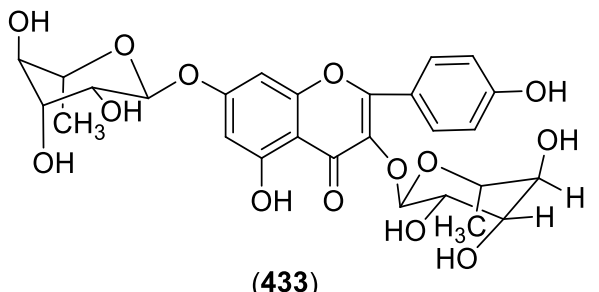

(433)<smiles>O=c1c(O)c(-c2ccc(O)cc2)oc2cc(O)cc(O)c12</smiles>

(434)<smiles>O=c1c(OC(O)C(O)C(O)C(O)C(O)O)c(-c2ccc(O)cc2)oc2cc(O)cc(O)c12</smiles>

(437)<smiles>O=c1c(OC2OC3C(O)C(CO)C(O)C3C2O)c(-c2ccc(O)c(O)c2)oc2cc(O)cc(O)c12</smiles>

(440)<smiles>O=c1c(OC(O)C(O)C(O)C(O)C(O)C(O)C(O)O)c(-c2ccc(O)cc2)oc2cc(O)cc(O)c12</smiles>

(435)<smiles>O=c1c(O)c(-c2ccc(O)c(O)c2)oc2cc(O)cc(O)c12</smiles>

(438)<smiles>O=c1c(OC(O)C(O)C(O)C(O)O)c(-c2ccc(O)c(O)c2)oc2cc(O)cc(O)c12</smiles>

(441)

Figure 4. Structures of compounds 432-441 from Cynanchum species. 


\subsection{Terpene}

The basic skeleton of terpenoids is a type of compound composed of isoprene structural units linked. There are two monoterpene diglycosides neohancoside A (442) and B (443) are monoterpene diglycosides isolated from $C$. hancockianum $A$ and $B$ [95]. In addition, there are also seven pentacyclic triterpene compounds $\beta$-amyrin (444), $\alpha$-amyrin (445), lupeol (446), taraxasterol (447), ursolic acid (448), oleanolic acid (449) and maslinic acid (450), were isolated from the roots of $C$. paniculatum [86]. The structures of these compounds are shown in Figure 5.

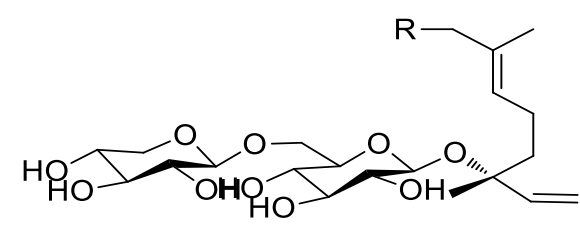

(442) $\quad \mathrm{R}=\mathrm{OH}$

(443) $\mathrm{R}=\mathrm{OH}$

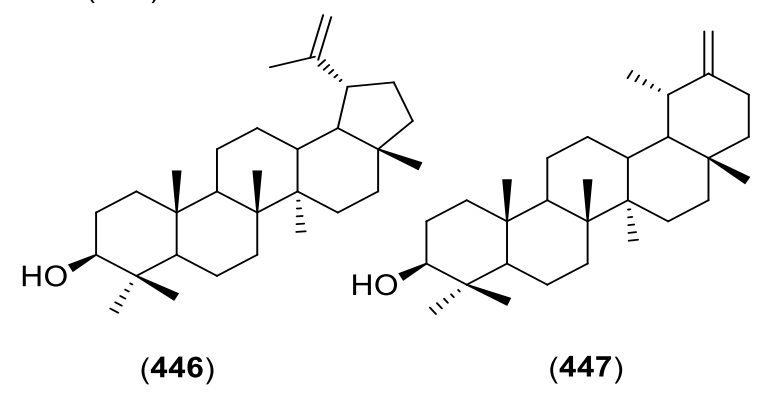

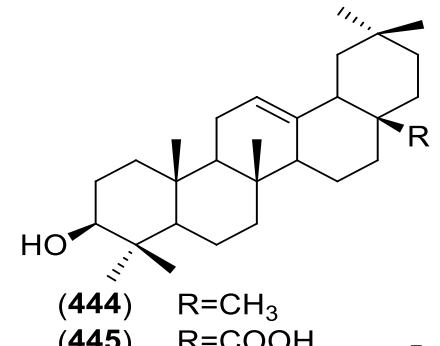

(445) $\mathrm{R}=\mathrm{COOH}$

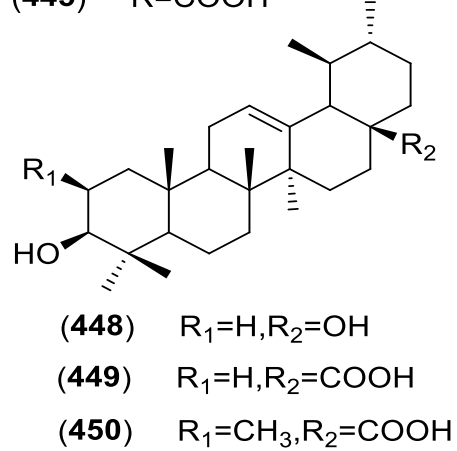

Figure 5. Structures of compounds 442-450 from Cynanchum species.

\subsection{Others}

In addition to the above-mentioned main components, other components, such as carboxylic acid, alcohol, ester and lignin, are foundin Cynanchum. These compounds include azelaic acid, suberic acid and succinic acid [85];3,3'-dimethoxy-4,9,9'-trihydroxy-benzofuranoid ligan-7'-ene-9-O- $\beta$ D-glucoside; 3,5-dihydroxybenzoic acid methyl ester; 4-dydroxybenzoic acid; 2,5-dihydroxybenzoic acid methyl ester [56]; conduritol F [3], p-menthane-1,7,8-triol, 1-p-menthane-8,9-triol, $p$-menthane-1,8,9-triol,trans-terpin [37], 2,6,2',6' ${ }^{\prime}$-tetramethoxy-4,4'-bis(2,3-epoxy-1-hydroxypropyl)biphenyl [39] and (+)-(7S,8R, $\left.7^{\prime} E\right)$-5-hydroxy-3, $5^{\prime}$-dimethoxy-4',7-epoxy-8, $3^{\prime}$-neolign-7'-ene-9, $9^{\prime}$-diol $9^{\prime}$-ethyl ether [63].

\section{Pharmacology}

In recent years, research reports on the chemical constituents and pharmacological activities of plants of genus Cynanchum have shown an increasing trend. An increasing number of researchers show special interest in this genus and its therapeutic properties in the field of traditional Chinese medicine. In Table 4, it was summarized on the major ethnic pharmacological uses of Cynanchum sp. and the status of modern pharmacological evaluation. Its pharmacological effects are mainly anti-cancer, anti-inflammatory, anti-virus, appetite suppressing and other effects. 
Table 4. Summary of pharmacological activities of the extracts/compounds from different parts of Cynanchum species.

\begin{tabular}{|c|c|c|c|c|c|c|}
\hline Cynanchum Species & Extract/Isolate & Plant Part & In Vitro/In Vivo & Dosage/Duration & Model/Effect & Ref \\
\hline \multicolumn{7}{|c|}{ Anti-cancer } \\
\hline C. taiwanianum & $\begin{array}{l}\text { Cynantetrone, } \\
\text { cynandione B }\end{array}$ & Rhizome & In vitro & & $\begin{array}{l}\text { Compounds against T-24 cell lines with } \\
\mathrm{ED}_{50} \text { values of ca. } 3.5 \text { and } 2.5 \mu \mathrm{g} / \mathrm{mL} \text {, } \\
\text { respectively, and cynandione } \mathrm{B} \text { against } \\
\text { PLC } / \mathrm{PRF} / 5 \text { cell lines }\left(\mathrm{ED}_{50}=2.7 \mu \mathrm{g} / \mathrm{mL}\right) \text {. }\end{array}$ & {$[78]$} \\
\hline \multirow[b]{2}{*}{ C.auriculatum } & \multirow{2}{*}{$\begin{array}{l}\text { Ethanol extract, } \\
\text { Petroleumether, } \mathrm{CHCl}_{3}, \\
\text { EtOAc and } n-\mathrm{BuOH} \\
\text { fraction }\end{array}$} & \multirow[b]{2}{*}{ Root tubers } & In vitro & $1 \mu \mathrm{g} / \mathrm{mL}$ & $\begin{array}{l}\text { The ethanol extract against K562, with the } \\
\text { highest inhibition ratio of } 24.06 \% \text { at a } \\
\text { concentration of } 1 \mu \mathrm{g} / \mathrm{mL} \text {. }\end{array}$ & \multirow[t]{2}{*}{96} \\
\hline & & & In vivo & $100 \mathrm{mg} / \mathrm{kg} /$ Gavage $7 \mathrm{~d}$ & $\begin{array}{l}\text { The ethanol extract and n-BuOH fraction } \\
\text { showed significant antitumor activity by } \\
\text { inhibiting the growth of sarcoma S180 in } \\
\text { mice with an inhibition ratio of } 42.22 \% \text { and } \\
41.50 \% \text {. }\end{array}$ & \\
\hline C. auriculatum & Total glucosides & & In vivo & $225 \mathrm{mg} / \mathrm{kg} 10 \mathrm{~d}$ & $\begin{array}{c}\text { Model: C57BL/ } 6 \text { mice bearing Lewis lung } \\
\text { carcinoma. The inhibition rate of tumor } \\
\text { weight was } 38.68 \% \text { the inhibition rate of } \\
\text { lung metastasis was } 63.64 \% \text {. }\end{array}$ & [97] \\
\hline \multirow[b]{2}{*}{ C.auriculatum } & \multirow{2}{*}{$\begin{array}{l}\text { Caudatin, } \\
\text { caudatin-2,6-dideoxy-3-O- } \\
\text { methy- } \beta \text {-D-cymaropyranoside }\end{array}$} & \multirow[t]{2}{*}{ Root tubers } & In vitro & $12 \mu \mathrm{M}$ & $\begin{array}{l}\text { Model: Human tumor cell line SMMC-7721. } \\
\qquad \mathrm{IC}_{50}=24.95 \mu \mathrm{M} ; \mathrm{IC}_{50}=13.49 \mu \mathrm{M}\end{array}$ & \multirow[t]{2}{*}{48} \\
\hline & & & In vivo & $10,20,40 \mathrm{mg} / \mathrm{Kg} 9 \mathrm{~d}$ & $\begin{array}{l}\text { Model: Transplantable } \mathrm{H} 22 \text { tumors in mice. } \\
\text { The growth of transplantable H22tumors in } \\
\text { mice was inhibited. }\end{array}$ & \\
\hline C.auriculatum & $\begin{array}{c}\text { Kidjoranin 3-O- } \alpha \text { - } \\
\text { diginopyranosyl- }(1 \rightarrow 4)-\beta- \\
\text { cymaropyranoside, } \\
\text { kidjoranin } \\
\text { 3-O- } \beta \text {-digitoxopyranoside, } \\
\text { caudatin } \\
\text { 3-O- } \beta \text {-cymaropyranoside }\end{array}$ & Roots & In vitro & & $\begin{array}{l}\text { Model: SMMC-7721 and HeLa cell lines. } \\
\qquad \mathrm{IC}_{50}=8.6 \mu \mathrm{M}-58.5 \mu \mathrm{M}\end{array}$ & {$[98]$} \\
\hline
\end{tabular}


Table 4. Cont

\begin{tabular}{|c|c|c|c|c|c|c|}
\hline Cynanchum Species & Extract/Isolate & Plant Part & In Vitro/In Vivo & Dosage/Duration & Model/Effect & Ref. \\
\hline C. auriculatum & $\begin{array}{l}\text { Auriculoside A, } \\
\text { auriculoside B }\end{array}$ & Roots & In vitro & & $\begin{array}{l}\text { Have significant cytoxicity against PC3, } \\
\text { Hce-8693, Hela, and PAA cell lines. }\end{array}$ & [99] \\
\hline C. vincetoxicum & Alkaloids & Overground & In vitro & & $\begin{array}{l}\text { These alkaloids inhibit growth of the } \\
\text { hormone in dependent breast cancer cells } \\
\text { MDA-MB } 231 \text {. }\end{array}$ & [88] \\
\hline C. paniculatum & $\begin{array}{l}\text { Neocynapanogenin } \mathrm{F}, \\
\text { neocynapanogenin } \mathrm{F} \\
\text { 3-O- } \beta \text {-D-thevetoside }\end{array}$ & Roots & In vitro & $100 \mu \mathrm{g} / \mathrm{mL}$ & $\begin{array}{l}\text { These compounds exhibited significant } \\
\text { cytotoxic activity on HL- } 60 \text {. The inhibitory } \\
\text { rate }(\%, n=6) \text { was } 74.18 \% \text { and } 97.87 \% \text {, } \\
\text { respectively. }\end{array}$ & [35] \\
\hline C. paniculatum & $\begin{array}{c}\text { Cynanside A, Cynanside } \\
\text { B }\end{array}$ & Roots & In vitro & & $\begin{array}{l}\text { Model: } \text { SK-MEL-2 cells. } \\
\text { IC }_{50} \text { values }=26.55 \mu \mathrm{M} \\
\text { IC }_{50} \text { values }=17.36 \mu \mathrm{M}\end{array}$ & [58] \\
\hline \multirow{2}{*}{ C. paniculatum } & \multirow{2}{*}{ Antofine } & \multirow[b]{2}{*}{ Roots } & \multirow{2}{*}{ In vitro } & $\begin{array}{c}\text { Ellipticine: } \mathrm{IC}_{50}=500 \pm \\
25 \mathrm{ng} / \mathrm{mL}\end{array}$ & $\begin{array}{l}\text { Model: Human lung cancer cells A549. } \\
\qquad \mathrm{IC}_{50}=7.0 \pm 0.2 \mathrm{ng} / \mathrm{mL}\end{array}$ & \multirow{2}{*}[100]{} \\
\hline & & & & $\begin{array}{c}\text { Ellipticine: } \mathrm{IC}_{50}=340 \pm \\
35 \mathrm{ng} / \mathrm{mL}\end{array}$ & $\begin{array}{l}\text { Model: Human colon cancer cells Col2. } \\
\mathrm{IC}_{50}=8.6 \pm 0.3 \mathrm{ng} / \mathrm{mL}\end{array}$ & \\
\hline \multirow{3}{*}{ C. wilfordii } & 20-O-salicyl-kidjoranin & \multirow{3}{*}{ Roots } & \multirow{3}{*}{ In vitro } & \multirow{3}{*}{ Adriamycin } & $\begin{array}{l}\text { Model: Human leukemia cell lines HL-60, } \\
\text { K562 and breast cancer cell lines MCF-7. } \\
\text { The compound can against HL-60 (IC } 50= \\
6.72 \mu \mathrm{M}) \text { and MCF-7 }\left(\mathrm{IC}_{50}=2.89 \mu \mathrm{M}\right) \text {. }\end{array}$ & \multirow{3}{*}{ [9] } \\
\hline & Qingyangshengenin & & & & $\begin{array}{l}\text { The compound can against K-562 }\left(\mathrm{IC}_{50}=\right. \\
\qquad 6.72 \mu \mathrm{M}) .\end{array}$ & \\
\hline & Rostratamin & & & & $\begin{array}{l}\text { The compound can against MCF-7 }\left(\mathrm{IC}_{50}=\right. \\
\qquad 2.49 \mu \mathrm{M}) .\end{array}$ & \\
\hline
\end{tabular}


Table 4. Cont

\begin{tabular}{|c|c|c|c|c|c|c|}
\hline Cynanchum Species & Extract/Isolate & Plant Part & In Vitro/In Vivo & Dosage/Duration & Model/Effect & Ref. \\
\hline C. wilfordii & $\begin{array}{c}\text { Gagaminin 3-O- } \beta \text {-D- } \\
\text { cymaropyranosyl- }(1 \rightarrow 4)-\beta \text {-D- } \\
\text { oleandropyranosyl- }(1 \rightarrow 4)-\beta \text {-D- } \\
\text { cymaropyranosyl- }(1 \rightarrow 4)-\beta \text {-D- } \\
\text { cymaropyranoside }\end{array}$ & Roots & In vitro & $1 \mu \mathrm{M}$ & $\begin{array}{l}\text { Model: KB-V1 and MCF7/ADR cells. } \\
\text { The compounds completely reverse the } \\
\text { multidrug-resistance of KB-V1 and } \\
\text { MCF7/ADR cells to Adriamycin, } \\
\text { vinblastine, and colchicine. }\end{array}$ & {$[54]$} \\
\hline C. atratum & $\begin{array}{l}\text { Glaucogenin C 3-O- } \beta \text {-D- } \\
\text { cymaropyranosyl- }(1 \rightarrow 4)-\alpha \text {-L- } \\
\text { diginopyranosyl- }(1 \rightarrow 4)-\beta \text {-D- } \\
\text { thevetopyranoside }\end{array}$ & Roots & In vitro & $\begin{array}{l}\text { Dexamethasone: } 10 \mu \mathrm{M} \\
\text { compound: } 30 \mu \mathrm{M}\end{array}$ & $\begin{array}{l}\text { Model: } 212 \text { cells, RAW } 264.7 \text { mouse } \\
\text { macrophage-like cell, N9 microglial cell. } \\
\text { ED } 50 \text { value of against } 212 \text { cells was } 0.96 \\
\mu \mathrm{g} / \mathrm{mL} \text { and significant inhibitory on TNF- } \alpha \\
\text { formation. }\end{array}$ & [39] \\
\hline C. vincetoxicum & $\begin{array}{c}(-)-10 \beta \text {-antofine } N \text {-oxide } \\
(-)-10 \beta \\
\text { 13a } \alpha-14 \beta \text {-hydroxyantofine } \\
N \text {-oxide }\end{array}$ & Aerial parts & In vitro & & $\begin{array}{l}\text { Model: drug-sensitive KB-3-1 cell line and } \\
\text { the multi-drug-resistant KB-V1 cell line. } \\
\qquad \mathrm{IC}_{50}=100 \mathrm{nM}\end{array}$ & [90] \\
\hline C. vincetoxicum & $\begin{array}{l}(-)-(R)-13 \mathrm{a} \alpha \text {-antofine, } \\
(-)-(R)-13 \mathrm{a} \alpha-6-O- \\
\text { desmethylantofine }\end{array}$ & Leaves & In vitro & & $\begin{array}{l}\text { Model: KB-3-1 and the KB-V1 cell line. } \\
\text { IC } 50 \text { values of } 7-17 \mathrm{nM}\end{array}$ & [91] \\
\hline \multirow{4}{*}{ C. saccatum } & Cynsaccatol E & \multirow{4}{*}{ Roots } & \multirow{4}{*}{ In vitro } & \multirow{4}{*}{ 5-FU and cisplatin } & $\begin{array}{l}\text { Model: HepG2 cell lines } \mathrm{IC}_{50}=49.18 \pm \\
5.67 \mu \mathrm{M} .\end{array}$ & \multirow{4}{*}{ [55] } \\
\hline & $\begin{array}{c}\text { Gagaminine 3-O- } \beta \text {-D- } \\
\text { oleandropyranosyl- }(1 \rightarrow 4)- \\
\beta \text {-D-oleandropyranosyl- } \\
(1 \rightarrow 4)-\beta \text {-D-cymaropyranoside } \\
\end{array}$ & & & & $\begin{array}{l}\text { Model: HepG2 and Hela cell lines. } \\
\mathrm{IC}_{50}=68.05 \pm 4.09 \mu \mathrm{M} \text { and } \mathrm{IC}_{50}=94.88 \pm \\
9.73 \mu \mathrm{M}\end{array}$ & \\
\hline & Cynsaccatol A & & & & $\begin{array}{l}\text { Model: U251 cell lines. } \mathrm{IC}_{50}=35.66 \pm 3.54 \\
\mu \mathrm{M} .\end{array}$ & \\
\hline & Cynsaccatol D & & & & $\begin{array}{c}\text { Model: U251 cell lines. } \mathrm{IC}_{50}=31.98 \pm 6.55 \\
\mu \mathrm{M}\end{array}$ & \\
\hline C. saccatum & $\begin{array}{l}\text { Glaucogenin } \\
\text { C-3-O- } \beta \text {-D-monothevetoside }\end{array}$ & $\begin{array}{l}\text { Whole fresh } \\
\text { plants }\end{array}$ & In vitro & $\begin{array}{c}\text { Cisplatin: } \mathrm{IC}_{50}=21.51 \\
\mu \mathrm{M}\end{array}$ & $\begin{array}{l}\text { The compound could induce HepG2 cell } \\
\text { apoptosis via a mitochondrial pathway and } \\
\text { IC } 50 \text { value of } 12.24 \mu \mathrm{M}\end{array}$ & [101] \\
\hline
\end{tabular}


Table 4. Cont

\begin{tabular}{|c|c|c|c|c|c|c|}
\hline Cynanchum Species & Extract/Isolate & Plant Part & In Vitro/In Vivo & Dosage/Duration & Model/Effect & Ref. \\
\hline C. paniculatum & Cynatratoside B & Roots & In vitro & 5-Fluorouracil & $\begin{array}{l}\text { Compound exhibited potent inhibitory } \\
\text { activities against HL-60, HT-29, PC-3 and } \\
\text { MCF-7 cell lines with IC } \mathrm{IC}_{50} \text { values of 8.3, 7.5, } \\
34.3 \text { and } 19.4 \mu \mathrm{M} \text {, respectively. }\end{array}$ & {$[102]$} \\
\hline C. atratum & $\mathrm{C}_{21}$ steroids & Roots & In vitro & Cisplatin $(25 \mu \mathrm{g} / \mathrm{mL})$ & $\begin{array}{l}\text { Model: HepG2, A549 cell lines. } \\
\text { Compounds 1-4 displayed obvious } \\
\text { cytotoxic activities against HepG2 cells with } \\
\mathrm{IC}_{50} \text { values ranging from } 10.19 \mu \mathrm{M} \text { to } 76.12 \\
\mu \mathrm{M} \text {. Compounds 1-3 also exhibited } \\
\text { cytotoxic effects in A549 cells with } \\
\text { IC } \text { C }_{50} \text { values of } 30.87-95.39 \mu \mathrm{M} \text {. }\end{array}$ & {$[103$} \\
\hline \multicolumn{7}{|c|}{ Neuroprotective effect } \\
\hline C. wilfordii & Cynandione A & Roots & In vitro & $50 \mu \mathrm{M}$. & $\begin{array}{l}\text { Model: Neurotoxicity induced by } \mathrm{H}_{2} \mathrm{O}_{2} \text { in } \\
\text { cultured cortical cells. } \\
\text { The compound could reduce neurotoxicity } \\
\text { induced by } \mathrm{H}_{2} \mathrm{O}_{2} \text {. }\end{array}$ & {$[104]$} \\
\hline C. atratum & $\begin{array}{c}\text { Cynatroside A, cynatroside B, } \\
\text { cynatroside C, cynascyroside } \\
\text { D }\end{array}$ & Roots & In vitro & $\begin{array}{c}\text { Velnacrine: } \mathrm{IC}_{50}=0.4 \\
\mu \mathrm{M} .\end{array}$ & $\begin{array}{c}\text { These compounds could inhibit } \\
\text { acetylcholinesterase activity. } \\
\mathrm{IC}_{50}=6.4 \mu \mathrm{M}, \mathrm{IC}_{50}=3.6 \mu \mathrm{M}, \mathrm{IC}_{50}=52.3 \\
\mu \mathrm{M}, \mathrm{IC}_{50}=52.9 \mu \mathrm{M} \text {, respectively. }\end{array}$ & [25] \\
\hline C. paniculatum & \multicolumn{2}{|c|}{ 2,3-dihydroxy-4-methoxyacetophenoneRoots } & In vitro & Trolox $(10 \mu \mathrm{M})$ & $\begin{array}{l}\text { Model: Glutamate-induced neurotoxicity in } \\
\text { HT22 cells. } \\
\text { Relatively effective protection of } 47.55 \% \text { (at } \\
10 \mu \mathrm{M} \text { ). }\end{array}$ & [81] \\
\hline C. atratum & Cynatroside B & Roots & In vivo & $\begin{array}{l}\text { Donepezil: } 0.032-3.2 \\
\text { mg/Kg body weight } \\
\text { i.p. }\end{array}$ & $\begin{array}{l}\text { The results showed that compound has both } \\
\text { anti-AchE and anti-amnesic activities. }\end{array}$ & {$[105$} \\
\hline
\end{tabular}


Table 4. Cont

\begin{tabular}{|c|c|c|c|c|c|c|}
\hline Cynanchum Species & Extract/Isolate & Plant Part & In Vitro/In Vivo & Dosage/Duration & Model/Effect & Ref. \\
\hline C. otophyllum & $\begin{array}{c}\text { Cynanotoside A, } \\
\text { cynanotoside B, } \\
\text { cynotophylloside H }\end{array}$ & Roots and stems & In vitro & & $\begin{array}{l}\text { Three oxidative stress models induced by } \\
\text { glutamate, } \mathrm{H}_{2} \mathrm{O}_{2} \text {, and homocysteic acid } \\
\text { (HCA), respectively, in a hippocampal } \\
\text { neuronal cell line HT22. } \\
\text { Compounds showed significant } \\
\text { dose-dependent protection to HCA-induced } \\
\text { cell death ranging from } 1 \text { to } 30 \mu \mathrm{M} \text {. }\end{array}$ & [71] \\
\hline C. otophyllum & $\begin{array}{l}\text { Otophylloside F, } \\
\text { otophylloside B }\end{array}$ & Roots & In vivo & $\begin{array}{c}\text { phenytoin sodium } \\
\text { showed a therapeutic } \\
\text { efficacy of } 66 \% \text { at } 300 \\
\mu \mathrm{M}\end{array}$ & $\begin{array}{l}\text { Model: Antiseizure-like locomotor activity } \\
\text { in the zebrafish bioassay model. } \\
\text { The otophylloside F at a } 300 \mu \mathrm{M} \\
\text { concentration showed a therapeutic efficacy } \\
\text { of } 55 \% \text {. The otophylloside B at } 100 \text { and } 200 \\
\mu \mathrm{M} \text { concentrations showed therapeutic } \\
\text { efficacies of } 77 \% \text { and } 90 \% \text {, respectively. }\end{array}$ & [16] \\
\hline C. wilfordii & $\begin{array}{c}\text { Cynawilfoside A, } \\
\text { cynauricoside A, wilfoside } \\
\text { C1N, wilfoside K1N and } \\
\text { cyanoauriculoside G }\end{array}$ & Roots & In vivo & Retigabine: $15.0 \mathrm{mg} / \mathrm{kg}$ & $\begin{array}{l}\text { Model: MES-induced mouse seizure model. } \\
\mathrm{ED}_{50} \text { values of } 48.5,95.3,124.1,72.3 \text {, and } \\
88.1 \mathrm{mg} / \mathrm{kg} \text {, respectively. }\end{array}$ & {$[50]$} \\
\hline C. otophyllum & Otophylloside B & Roots & In vivo & Curcumin: $100 \mu \mathrm{M}$ & $\begin{array}{l}\text { Model: AD (Alzheimer's disease). } \\
50 \mu \mathrm{M}\end{array}$ & [106] \\
\hline \multicolumn{7}{|c|}{ Antifungal ,parasitic and antiviral Activity } \\
\hline C. wilfordii & $\begin{array}{l}\text { Wilfoside C1N, wilfoside } \\
\text { C1G, wilfoside C1GG }\end{array}$ & Roots & In vivo & $\begin{array}{c}\text { PolyoxinB }\left(\mathrm{IC}_{50} \text { value }=\right. \\
\quad 71.36 \mu \mathrm{g} / \mathrm{mL})\end{array}$ & $\begin{array}{l}\text { Model: Barley powdery mildew. } \\
\text { The IC } \text { IC }_{50} \text { (i.e., the concentration required for } \\
50 \% \text { inhibition) were determined as } 3.24 \\
\mu \mathrm{g} / \mathrm{mL}, 12.90 \mu \mathrm{g} / \mathrm{mL} \text {, and } 28.35 \mu \mathrm{g} / \mathrm{mL} \\
\text { respectively. }\end{array}$ & [27] \\
\hline C. paniculatum & Ethyl acetate (EA) extracts & Roots & In vitro & Amantadine & $\begin{array}{l}\text { Model: Madin-Darby bovine kidney } \\
\text { (MDBK) cells. } \\
\text { The tissue culture infectious dose assay } \\
\text { (TCID } 50 \text { ) assay. } \\
\text { The cytotoxic concentration C } C_{50} \text { was } 18.2 \\
\mu \mathrm{g} / \mathrm{mL} \text {; The EA MNTD (Maximum } \\
\text { non-toxic dose) is } 18.2 \mu \mathrm{g} / \mathrm{mL} \text {. }\end{array}$ & [107] \\
\hline
\end{tabular}


Table 4. Cont.

\begin{tabular}{|c|c|c|c|c|c|c|}
\hline Cynanchum Species & Extract/Isolate & Plant Part & In Vitro/In Vivo & Dosage/Duration & Model/Effect & Ref. \\
\hline C. atratum & Cynatratoside $\mathrm{C}$ & Roots & In vitro & & $\begin{array}{l}\text { Model: Grasscarp infected with I. multifiliis. } \\
\qquad 0.25 \mathrm{mg} / \mathrm{L} .\end{array}$ & [10] \\
\hline C. paniculatum & $\begin{array}{l}\text { Cynatratoside A; } \\
\text { cynanversicoside } \mathrm{C}\end{array}$ & Roots & In vitro & & $\begin{array}{l}\text { Cynatratoside A and cynanversicoside C } \\
\text { could be } 100 \% \text { effective against } I \text {. multifiliis } \\
\text { at the concentration of } 10.0 \mathrm{mg} \mathrm{L}^{-1} \text {, with } \\
\text { the median effective concentration }\left(\mathrm{EC}_{50}\right) \\
\text { values of } 4.6 \text { and } 5.2 \mathrm{mgL}^{-1} \text {, respectively. }\end{array}$ & [11] \\
\hline C. paniculatum & Essential oil & Roots & In vitro & $\begin{array}{l}\text { Benzyl benzoate and } \\
\text { DEET } \\
\text { (diethylmethylbenzamide) } \\
1.13 \mu \mathrm{g} / \mathrm{cm}^{2}\end{array}$ & $\begin{array}{c}\mathrm{LD}_{50} \text { were } 8.93,4.58, \text { and } 2.79 . \text { It showed } \\
\text { more toxic than DEET }\left(\mathrm{LD}_{50}=4.13,3.91\right. \\
\left.\text { and } 4.87 \mu \mathrm{g} / \mathrm{cm}^{2}\right) \text { against } \mathrm{D} \text {. farinae, } \mathrm{D} \text {. } \\
\text { pteronyssinus, and T. putrescentiae, } \\
\text { respectively. }\end{array}$ & [108] \\
\hline C. komarovii & $\begin{array}{l}\text { 7-demethoxytylophorine(1),7- } \\
\text { demethoxytylophorine } \\
\text { N-oxide(2) }\end{array}$ & Roots & In vitro & $\begin{array}{c}\text { 2,4-dioxo-hexahydro- } \\
\text { 1,3,5-triazine, showed } \\
50 \% \text { inhibition at } 500 \\
\mu \mathrm{g} / \mathrm{mL}\end{array}$ & $\begin{array}{l}\text { The alkaloid } 1 \text { exhibited } 65 \% \text { inhibition } \\
\text { against the TMV at a concentration of } 1.0 \\
\mu \mathrm{g} / \mathrm{mL} \text {. Alkaloid } 2 \text { showed } 60 \% \text { inhibition } \\
\text { at } 500 \mu \mathrm{g} / \mathrm{mL}\end{array}$ & [92] \\
\hline C. atratum & $\begin{array}{c}\text { Cynanoside A,G,M; } \\
\text { glaucogenin-C 3-O- } \\
\beta \text {-D-cymaropyranosyl- } \\
(1 \rightarrow 4)-\alpha \text {-L-diginopyranosyl- } \\
(1 \rightarrow 4)-\beta \text {-D-cymaropyranoside; } \\
\text { glaucogenin-A 3-O- } \\
\beta \text {-D-cymaropyranosyl- } \\
(1 \rightarrow 4)-\alpha \text {-L-diginopyranosyl- } \\
(1 \rightarrow 4)-\beta \text {-D-cymaropyranoside }\end{array}$ & Roots & In vivo & $\begin{array}{l}\text { Ningnanmycin }\left(\mathrm{IC}_{50}=\right. \\
49.6 \mu \mathrm{g} / \mathrm{mL})\end{array}$ & $\begin{array}{c}\mathrm{IC}_{50}=20.5 \mu \mathrm{g} / \mathrm{mL}, \mathrm{IC}_{50}=18.6 \mu \mathrm{g} / \mathrm{mL}, \mathrm{IC}_{50} \\
=22.0 \mu \mathrm{g} / \mathrm{mL}, \mathrm{IC}_{50}=19.2 \mu \mathrm{g} / \mathrm{mL}, \mathrm{IC}_{50}= \\
22.2 \mu \mathrm{g} / \mathrm{mL} \text {, respectively. }\end{array}$ & [36] \\
\hline C. stauntonii & Volatile oil & Roots & In vitro & $300 \mathrm{mg} / \mathrm{kg} 6 \mathrm{~d}$ & $\begin{array}{c}\text { Model: Mouse influenza model. } \mathrm{IC}_{50}=64 \\
\mu \mathrm{g} / \mathrm{mL}\end{array}$ & [109] \\
\hline
\end{tabular}


Table 4. Cont

\begin{tabular}{|c|c|c|c|c|c|c|}
\hline Cynanchum Species & Extract/Isolate & Plant Part & In Vitro/In Vivo & Dosage/Duration & Model/Effect & Ref. \\
\hline \multicolumn{7}{|c|}{ Immunosuppressive activity } \\
\hline C. chekiangense & $\begin{array}{l}\text { Chekiangensosides } \mathrm{A}, \\
\text { cynajapogenin } \mathrm{A}, \\
\text { chekiangensoside } \mathrm{B}, \\
\text { glaucogenin } \mathrm{A}\end{array}$ & Roots & In vitro & cyclosporin A & $\begin{array}{l}\text { Model: Con A- and LPS-induced } \\
\text { proliferation of mice splenocytes. } \\
\qquad 100 \mu \mathrm{L}(0.01-10 \mathrm{~g} / \mathrm{mL})\end{array}$ & [23] \\
\hline C. atratum & $\begin{array}{l}\text { Atratcynoside } \mathrm{A}, \\
\text { atratcynoside } \mathrm{B}, \\
\text { atratcynoside } \mathrm{C}\end{array}$ & Roots & In vitro & $\begin{array}{l}\text { Cyclosporin A: } 0.09 \pm \\
0.01 \mu \mathrm{M}\end{array}$ & $\begin{array}{c}\text { Model: Con A-induced proliferation of } \\
\text { T-lymphocytes from mice. } \\
\mathrm{IC}_{50} \text { values of } 3.3 \mu \mathrm{M}, 7.0 \mu \mathrm{M}, 6.7 \mu \mathrm{M}, \\
\text { respectively. }\end{array}$ & [72] \\
\hline \multicolumn{7}{|c|}{ Anti-inflammatory activity } \\
\hline C. stauntonii & $\begin{array}{l}\text { Cynastauoside B; } \\
\text { cynastauoside C }\end{array}$ & Roots & In vitro & $\begin{array}{c}\text { Dexamethasone with } \\
\text { the inhibition ratio of } \\
83.5 \% \text { at a } \\
\text { concentration of } 1 \mu \mathrm{M} .\end{array}$ & $\begin{array}{l}\text { Model: C57bl/6j mouse peritoneal } \\
\text { macrophages. } \\
\text { The results showed } 17.0 \% \text { and } 6.9 \% \text { of } \\
\text { inhibition rate at a concentration of } 10 \mu \mathrm{M} \text {, } \\
\text { respectively. }\end{array}$ & {$[74]$} \\
\hline C. wilfordii. & Cynandione A & Roots & In vitro & & $\begin{array}{l}\text { Model: LPS-Induced BV-2 microglial cells. } \\
\qquad \mathrm{IC}_{50}=27.13 \pm 5.38 \mu \mathrm{M}\end{array}$ & [110] \\
\hline C. stauntonii & $\begin{array}{c}\text { Stauntoside V1; stauntoside } \\
\text { V3 }\end{array}$ & Roots & In vitro & $\begin{array}{c}\text { Dexamethasone: } \mathrm{IC}_{50}= \\
0.3 \mu \mathrm{M}\end{array}$ & $\begin{array}{l}\text { Model: C57bl/6j mouse peritoneal } \\
\text { macrophages. } \\
\text { IC }_{50} \text { values of } 9.3 \mu \mathrm{M} \text { and } 12.4 \mu \mathrm{M} \text {, } \\
\text { respectively. }\end{array}$ & [111] \\
\hline \multirow{2}{*}{ C. atratum } & \multirow{2}{*}{ Aqueous extract } & \multirow{2}{*}{ Roots } & In vivo & \multirow{2}{*}{ dexamethasone } & Model: Female BALB/c mice/atopic & \multirow{2}{*}{ [112] } \\
\hline & & & In vitro & & $\begin{array}{l}\text { Dermatitis (AD) and Human mast cell line } \\
(\mathrm{HMC}-1) .1 \text { or } 100 \mathrm{mg} / \mathrm{mL} \text {. }\end{array}$ & \\
\hline \multirow{2}{*}{ C. wilfordii } & \multirow{2}{*}{ Polysaccharides } & \multirow{2}{*}{ Roots } & In vivo & $\begin{array}{l}\text { 5-aminosalicylic acid } \\
\quad(100 \mathrm{mg} / \mathrm{kg})\end{array}$ & $\begin{array}{l}\text { Model: DSS (dextran sodium } \\
\text { sulfate)-induced chroniccolitis in mice. } \\
200 \mathrm{mg} / \mathrm{kg} \text { or } 100 \mathrm{mg} / \mathrm{kg}\end{array}$ & \multirow{2}{*}{ [113] } \\
\hline & & & In vitro & & $\begin{array}{l}\text { Model: LPS-induced RAW } 264.7 \\
\text { macrophages. } \\
25 \mu \mathrm{g} / \mathrm{mL}\end{array}$ & \\
\hline
\end{tabular}


Table 4. Cont

\begin{tabular}{|c|c|c|c|c|c|c|}
\hline Cynanchum Species & Extract/Isolate & Plant Part & In Vitro/In Vivo & Dosage/Duration & Model/Effect & Ref. \\
\hline \multicolumn{7}{|c|}{ Anti-oxidation } \\
\hline C. wilfordi & Gagaminine & Roots & In vivo & $\begin{array}{c}\text { Pyridoxal: } \mathrm{IC}_{50}=246 \\
\mu \mathrm{M}\end{array}$ & $\begin{array}{l}\text { Model: Rat liver injury model. } \\
\mathrm{IC}_{50}=0.8 \mu \mathrm{M}(0.5 \mu \mathrm{g} / \mathrm{mL})\end{array}$ & [94] \\
\hline C. otophyllum & Otophyllosides A and B & Roots & In vivo & & $\begin{array}{l}\text { These compounds could protect rats from } \\
\text { audiogenic seizures and ED } \mathrm{D}_{50} \text { value of } 10.2 \\
\mathrm{mg} / \mathrm{kg} \text {. }\end{array}$ & [8] \\
\hline \multicolumn{7}{|c|}{ Hepatoprotective activity } \\
\hline C. wilfordii & Cynandione A & Roots & In vitro & Silybin $(100 \mu \mathrm{M})$ & $\begin{array}{l}\text { Model: Primary cultures of rat hepatocytes } \\
\text { injured by } \mathrm{CCl}_{4} \text {. } \\
50 \mu \mathrm{M}\end{array}$ & [114] \\
\hline C. wilfordii & Crude extract (CWE) & Roots & In vivo & $\begin{array}{c}\text { Simvastatin } / 10 \\
\mathrm{mg} / \mathrm{kg} / \text { day } / 12 \text { weeks } \\
\text { CWE: } 100 \text { and } 200 \\
\mathrm{mg} / \mathrm{kg} / \text { day } / 12 \text { weeks }\end{array}$ & $\begin{array}{l}\text { Model: Male C57BL/ } 6 \text { mice. } \\
\text { CWE can inhibit fat accumulation in the } \\
\text { liver. Suppressing lipid accumulation in the } \\
\text { liver and reducing blood levels of total } \\
\text { cholesterol and triglycerides. }\end{array}$ & [115] \\
\hline \multicolumn{7}{|c|}{ Appetite suppressant effect } \\
\hline C.auriculatum & Wilfoside K1N & Roots & In vivo & $\begin{array}{c}\text { Sibutramine } \\
15 \mathrm{mg} / \mathrm{kg} \text { body weight } \\
\text { Compound: } 50 \mathrm{mg} / \mathrm{kg} \\
\text { body weight }\end{array}$ & Model: SPF female Wistar rats. & {$[30]$} \\
\hline \multicolumn{7}{|c|}{ Antidepressant activity } \\
\hline C. auriculatum & $\begin{array}{l}\text { Cynanauriculoside C, } \\
\text { cynanauriculoside D, } \\
\text { cynanauriculoside E, } \\
\text { otophylloside L, } \\
\text { cynauricuoside C }\end{array}$ & Roots & In vivo & $\begin{array}{l}\text { fluoxetine }(20 \mathrm{mg} / \mathrm{kg}) \\
\text { Compound: } 50 \mathrm{mg} / \mathrm{kg} \\
\text { (i.g.)/twice a day } / 5 \mathrm{~d} \\
\text { Male ICR mice }(18-22 \\
\text { g) }\end{array}$ & $\begin{array}{l}\text { These compounds could significant } \\
\text { antidepressant activity at the dosage of } 50 \\
\mathrm{mg} / \mathrm{kg} \text { (i.g.) }\end{array}$ & {$[67]$} \\
\hline \multicolumn{7}{|c|}{ Vasodilating activity } \\
\hline C. stauntonii & Stauntonine & Roots & In vivo & & $\mathrm{IC}_{50}=5.37 \times 10^{-6} \mathrm{~mol} / \mathrm{L}$ & {$[40]$} \\
\hline
\end{tabular}


Table 4. Cont

\begin{tabular}{|c|c|c|c|c|c|c|}
\hline Cynanchum Species & Extract/Isolate & Plant Part & In Vitro/In Vivo & Dosage/Duration & Model/Effect & Ref. \\
\hline C. auriculatum & Caudatin & & $\begin{array}{l}\text { In vitro and } \\
\text { In vivo }\end{array}$ & & $\begin{array}{l}\text { Model: HUVEC human umbilical vein } \\
\text { endothelial cell and U251 human glioma } \\
\text { cells xenograft model. } \\
25-200 \mu \mathrm{M} \text {. }\end{array}$ & [116] \\
\hline \multicolumn{7}{|c|}{ Others } \\
\hline C. bungei & $\begin{array}{c}\text { 2,5-dihydroxyacetophenone } \\
\text { (2,5-DHAP) }\end{array}$ & Roots & $\begin{array}{l}\text { In vitro and } \\
\text { In vivo }\end{array}$ & $\begin{array}{l}\text { Standard depigmenting } \\
\text { agent: } 0.2 \mathrm{mM}\end{array}$ & $0.4 \mathrm{mM}$ & [79] \\
\hline C. stauntonii & Stauntosaponins A and B & Roots & In vitro & $\begin{array}{l}\text { Ouabain: } \mathrm{IC}_{50} \text { value of } \\
3.5 \mu \mathrm{M} \text {. Assay of } \\
\mathrm{Na}+/ \mathrm{K}+-\mathrm{ATP} \text { ase } \\
\text { inhibition }\end{array}$ & $\mathrm{IC}_{50}=21 \mu \mathrm{M}$ and $\mathrm{IC}_{50}=29 \mu \mathrm{M}$ & [77] \\
\hline \multirow[t]{2}{*}{ C. taiwanianum } & Cynandione B & Plants & In vitro & & $\begin{array}{c}\text { Model: The } \\
\text { formyl-methionyl-leucyl-phenylalanine } \\
\text { (fMLP)-stimulated rat neutrophil washed } \\
\text { rabbit platelets induced by arachidonic acid. } \\
\text { IC }_{50}=1.5 \pm 0.2 \text { and } 1.6 \pm 0.2 \mu \mathrm{M} \\
\text { respectively. }\end{array}$ & [117] \\
\hline & 2,5-Dihydroxyacetophenone & & & & $\mathrm{IC}_{50}=4.8 \mu \mathrm{M}$ & \\
\hline C. stauntonii & Cynatratoside B & Roots & In vitro & $\begin{array}{c}\text { Isoprenaline: } \mathrm{IC}_{50}= \\
0.13 \mu \mathrm{M}\end{array}$ & $\begin{array}{c}\text { Model: Rat Tracheal Rings Preparation. } \\
\text { The } \mathrm{EC}_{50} \text { acetylcholine- and } \\
\text { carbachol-induced contraction of compound } \\
\text { were } 0.67 \text { and } 0.38 \mu \mathrm{g} / \mathrm{mL}(\sim 0.85 \text { and } 0.48 \\
\mu \mathrm{M}) \text {, respectively. }\end{array}$ & [12] \\
\hline
\end{tabular}




\subsection{Anti-Cancer}

Crude extracts and compounds have significant activity against tumor cells, such as the SMMC-7721, MCF-7, Hela, K562, SHG44, HCT-8, A549, PC3, PLC/PRF/5, KB, T-24, A549, SK-OV-3, SK-MEL-2, HCT-15, Col2, 212, HepG2 and U251 cell lines in vitro. However, few studies have been conducted on the anti-cancer activity of Cynanchum plants in vivo.

The anti-cancer activity of the ethanol extract of $C$. auriculatum and different solvent extraction fractions was studied by inhibiting the growth of sarcoma S180 in mice and In vitro MTT assay. The ethanol extract inhibits K562 cell growth, with the highest inhibition ratio of $24.06 \%$ at a concentration of $1 \mu \mathrm{g} / \mathrm{mL}$ [96]. The inhibition rate of petroleum ether to PC3 cells at a concentration of $100 \mu \mathrm{g} / \mathrm{mL}$ is $33.63 \%$. At a concentration of $100 \mu \mathrm{g} / \mathrm{mL}$, the inhibition ratio of the $\mathrm{CHCl}_{3}$ fraction against K562, SHG44, HCT-8, A549 and PC3 are 35.64\%, 20.61\%, 31.64\%, 26.99\% and 52.11\%, respectively. The inhibitory rates of EtOAc fraction on A549 and PC 3 cells are $37.86 \%$ and $28.41 \%$, respectively. The $n-\mathrm{BuOH}$ fraction shows weak cytotoxicity to other cells at the same concentration except for K562 cells. In addition, the ethanol extract and $n$ - $\mathrm{BuOH}$ fraction inhibit the growth of sarcoma S180 in mice compared with the blank control $(p<0.01)$ at a dose of $100 \mathrm{mg} / \mathrm{kg}$.

Compounds 389 and 392 from the rhizomes of $C$. taiwanianum showed significant cytotoxic effects against T-24 cell lines with $\mathrm{ED}_{50}$ values of ca. 3.5 and $2.5 \mathrm{mg} / \mathrm{mL}$, respectively. Compound 385 also adversely affected PLC/PRF $/ 5$ cell lines $\left(\mathrm{ED}_{50}=2.7 \mathrm{mg} / \mathrm{mL}\right)[78]$.

In 1992, alkaloids 420-423 extracted from C. vincetoxicum, were found to inhibit the growth of MDA-MB-231 mammary carcinoma cells. Compounds 420, 425-429 and 430, which were isolated from the aerial parts of $C$. vincetoxicum, are assessed In vitro using both drug-sensitive KB-3-1 and multidrug-resistant KB-V1 cancer cell lines [90,91]. The results showed that compounds 420, 425, 427 and 430 exhibited pronounced cytotoxicity against KB-3-1 and KB-V1 cell lines with $\mathrm{IC}_{50}$ (the concentration required for $50 \%$ inhibition) values in the low nanomolar range. In addition, Sang et al. found that compound 420, which is isolated from the root of C. paniculatum, inhibits the growth of A549 and Col2 cell lines with $\mathrm{IC}_{50}$ values of $7.0 \pm 0.2$ and $8.6 \pm 0.3 \mathrm{ng} / \mathrm{mL}$ [100]. Ellipticine, as a positive control, exhibited $\mathrm{IC}_{50}$ value for A549 and Col2 cancer cells ranging from 300-500 ng/mL. Moreover, Col2 cells considerably accumulate in the G2/M cell cycle when treated with antofine $(50 \mathrm{pg} / \mathrm{mL})$ for $48 \mathrm{~h}$. Therefore, this mechanism may be the main process by which antofine inhibits the growth of Col2 cells [100].

Compound 215 was isolated from the roots of $C$. wilfordii (CWW) and completely reverse the multidrug resistance of KB-V1 and MCF7/ADR cells to Adriamycin, vinblastine and colchicine at a concentration level of $1 \mu \mathrm{M}$ [54].The inhibitory ratio of compound $\mathbf{1 1 6}$ isolated from ethyl acetate extract of C. paniculatum to HL-60 cells at a concentration of $10 \mu \mathrm{g} / \mathrm{mL}$ is $98.14 \%$ [35]. Kim et al. evaluated the anti-cancer activity of compounds 232 and 233 isolated from the roots of C. paniculatum against A549, SK-OV-3, SK-MEL-2 and HCT-15 cell lines In vitro by using the SRB bioassay [58]. Experimental results showed that compounds 232 and 233 have selective cytotoxicity on SK-MEL-2 cells with $\mathrm{IC}_{50}$ values of 26.55 and $17.36 \mu \mathrm{M}$, respectively.

C21 steroidal compounds, which isolated from genus Cynanchum also exhibit strong anti-cancer activity. Compound $\mathbf{1 2 0}$ isolated from the roots of CA showed significant cytotoxic effect against 212 cells, with $\mathrm{ED}_{50}$ value of $0.96 \mu \mathrm{g} / \mathrm{mL}$ [39].

Two C21 steroidal glycosides, namely, compounds $\mathbf{1 7 5}$ and $\mathbf{1 7 6}$ that were isolated from the roots of C. auriculatum are tested on SMMC-7721, MCF-7 and Hela cell lines. The results showed that the $\mathrm{IC}_{50}$ values of the two compounds against SMMC-7721 cells are 13.49 and $24.95 \mu \mathrm{M}$, respectively. Then, the in vivo assay by using solid tumor model H22 in mice was performed [48]. It was found that compounds $\mathbf{1 7 5}$ and $\mathbf{1 7 6}$ can significantly inhibit the growth of transplantable H22 tumors in mice at doses of 10, 20, and $40 \mathrm{mg} / \mathrm{kg}$ compared with positive control 5-FU.

The anti-cancer activities of 17 C21-steroidal pregnane sapogenins, namely, compounds 8, 167, 170-172, 174, 175, 177, 200, 209-212, 221, 223, 228 and 417, were evaluated by activity using HL-60, K-562 and MCF-7 cancer cells [9]. The results suggested that compound 8 shows evident cytotoxicity on HL-60 
$\left(\mathrm{IC}_{50}=6.72 \mu \mathrm{M}\right)$ and MCF-7 cell lines $\left(\mathrm{IC}_{50}=2.89 \mu \mathrm{M}\right)$, whereas compounds 200 and 221 show strong inhibitory activities against K-562 $\left(\mathrm{IC}_{50}=6.72 \mu \mathrm{M}\right)$ and MCF-7 cell lines $\left(\mathrm{IC}_{50}=2.49 \mu \mathrm{M}\right)$, respectively.

Zhang et al. [46] studied the anti-cancer activity of 26 pregnane glycosides (compounds 37, 38, 43, 168, 184-195, 204-207, 214, 220, 323, 325, 368 and 369) by using three cancer cells (HepG2, Hela and U251). All of these pregnane glycosides compared with the positive compounds 5-FU and cisplatin showed cytotoxic activities $\left(\mathrm{IC}_{50}<100 \mu \mathrm{M}\right)$ in varying degrees against these cell lines except compounds 189 and $205\left(\mathrm{IC}_{50}>100 \mu \mathrm{M}\right)$. Moreover, the cytotoxicity of compounds 38, 219, 310-317 is evaluated against three human cancer cell lines, that is, HepG2, Hela and U251 [55].

\subsection{Neuroprotective Effect}

With the development of the aging population, the incidence of the neurodegenerative diseases also shows a clear upward trend [118]. Therefore, the mechanisms of prevention and early treatment of these diseases have become one of the focuses of research. Research showed that numerous compounds isolated from genus Cynanchum exhibit good neuroprotective effects, thereby indicating its potential for further development.

Compound 391 can protect cultured cortical neurons from toxicity induced by $\mathrm{H}_{2} \mathrm{O}_{2}$, L-glutamate and kainate. Compound 391 showed the most potent neuroprotective activity at a concentration of $50 \mu \mathrm{M}$. Given its significant neuroprotective effect on cultured cortical neurons, the compound can effectively protect the neurons from oxidative stress mediated by activating a-amino-3-hydroxy-5-methyl-4-isoxazolepropionate/kainate receptors [104].

The inhibitory activities of compounds $85-87$ and 278 were tested against acetylcholinesterase (AChE). The result showed that compounds 85 and 86 exhibit the most potent inhibitory activity against AchE, with $\mathrm{IC}_{50}$ values of ca.6.4 and $3.6 \mu \mathrm{M}$, respectively. Compounds 87 and 278 also show AChE inhibition activity, with $\mathrm{IC}_{50}$ values of ca. 52.3 and $152.9 \mu \mathrm{M}$, respectively [25]. In addition, the anti-amnesic activity of compound $\mathbf{8 6}$ was investigated in passive avoidance and Morris water maze tests [105]. The results showed that compound 86 (1.0 mg/kg body weight i.p.) has significantly ameliorated the memory impairments induced in mice by scopolamine $(1.0 \mathrm{mg} / \mathrm{kg}$ body weight s.c.).

The neuroprotective effect of compound 398 against glutamate-induced neurotoxicity in mouse hippocampal HT22 cells was investigated; the result revealed that this compound exerts a neuroprotective effect on glutamate-induced neurotoxicity in HT22 cells, with relatively effective protection of $47.55 \%$ at $10 \mu \mathrm{M}$ [81]. In the hippocampal neuronal cell line HT22, compounds 363, 364 and 322 resist HCA-induced neuronal cell death within a concentration range of $1-30 \mu \mathrm{M}$ in a concentration-dependent manner [71].

The effects of 19 compounds which have C21 steroidal structure on anti-seizure-like locomotor activity caused by pentylenetetrazole in zebrafish model were also evaluated. The results showed that compounds 30, 28 and 223 exert a significant therapeutic effect on epilepsy. The results revealed that compound 30 has a therapeutic efficacy of $55 \%$ at a concentration of $300 \mu \mathrm{M}$, whereas compound 28 shows therapeutic efficacies of $77 \%$ and $90 \%$ at 100 and $200 \mu \mathrm{M}$ concentrations, respectively. Meanwhile, compound 223 showed therapeutic efficacies of $65 \%$ and $52 \%$ at 100 and $200 \mu \mathrm{M}$ concentrations, respectively. In comparison, the positive control, phenytoin sodium, shows $66 \%$ therapeutic efficacy at a concentration of $300 \mu \mathrm{M}$. The results also suggested that these three compounds do not exert any nonspecific neurotoxic or sedative effects or affect locomotor activity [16].

In addition, the anti-epileptic activity of $10 \mathrm{C} 21$ steroidal compounds were evaluated by Li et al. by using the mouse maximal electroshock (MES) model after oral administration. The results suggested that five compounds, namely, compounds 326, 240, 99, 96 and 302, exhibit significant protection activity in a MES-induced mouse seizure model, with $\mathrm{ED}_{50}$ values of $48.5,95.3,124.1,72.3$ and $88.1 \mathrm{mg} / \mathrm{kg}$, respectively. Under identical experimental conditions, the $\mathrm{ED}_{50}$ value of the positive control retigabine is $15.0 \mathrm{mg} / \mathrm{kg}$ [50]. 


\subsection{Anti-Fungal, Anti-Parasitic and Anti-Viral Activities}

In the recent years, both compounds and the crude extracts, such as volatile oil and ethyl acetate extracts, from CWW, CA, C. komarovii and other plants were investigated for their anti-fungal, parasitic or anti-viral activity, as shown below.

Six compounds, namely, compounds 96-99, 103 and 230 isolated from CWW roots, were evaluated against barley powdery mildew In vivo and compared with the anti-fungal activity of polyoxin B. The results suggested that compounds 98, 99 and 103 exhibit potent In vivo anti-fungal activities and present disease-control values of $>77 \%$ at a concentration of $63 \mu \mathrm{g} / \mathrm{mL}$. The $\mathrm{IC}_{50}$ values (the concentration required for $50 \%$ inhibition) are 3.24, 12.90, and $28.35 \mu \mathrm{g} / \mathrm{mL}$ for compounds 99,103 and 98 , respectively [27].

Compound 20 was isolated from CA roots and was used to treat Ichthyophthirius multifiliis. This compound demonstrates $100 \%$ mortality rate of $I$. multifiliis in vitro after $5 \mathrm{~h}$ of exposure at $0.25 \mathrm{mg} / \mathrm{L}$. The 5-hmedian effective concentration of compound to non-encysted tomonts is $0.083 \mathrm{mg} / \mathrm{L}$ [10].

Compounds 431-433 exhibit inhibitory activities against Tobacco mosaic virus (TMV). The results showed that alkaloids $\mathbf{4 3 2}$ and $\mathbf{4 3 3}$ exhibit anti-viral activities against TMV. The major active ingredient 432 exhibits $65 \%$ inhibition against the TMV at a concentration of $1.0 \mathrm{mg} / \mathrm{mL}$. Alkaloid 433 shows $60 \%$ inhibition at $500 \mathrm{mg} / \mathrm{mL}$, whereas compound 431 shows $15 \%$ inhibition at $500 \mathrm{mg} / \mathrm{mL}$ [92]. In comparison, 2,4-dioxo-hexahydro-1,3,5-triazine shows 50\% inhibition at $500 \mathrm{mg} / \mathrm{mL}$ under the same conditions.

In addition, Yan et al. studied the anti-TMV activities of 42 compounds isolated from the roots of CA by using the conventional half-leaf method, enzyme-linked immunosorbent assay, and Western blot [36]. The results suggested that compounds 52, 58, 64, 127 and 135 show significant anti-TMV activities with $\mathrm{IC}_{50}$ values of 20.5, 18.6, 22.0, 19.2 and $22.2 \mu \mathrm{g} / \mathrm{mL}$, respectively. Moreover, the anti-TMV activities of these compounds are considerably more effective than that of the positive control, ningnanmycin $\left(\mathrm{IC}_{50}=49.6 \mu \mathrm{g} / \mathrm{mL}\right)$.

The ethyl acetate extract of C. paniculatum exert an anti-viral effect against Bovine viral diarrhea (BVD) virus. The cytotoxic concentration $\left(\mathrm{CC}_{50}\right.$ for the ethyl acetate extracts is $18.2 \mu \mathrm{g} / \mathrm{mL}$. In the tissue culture infectious dose assay, the BVD virus decreased when treated with $18.2 \mu \mathrm{g} / \mathrm{mL}$ of the ethyl acetate extracts [107].

\subsection{Anti-Inflammatory and Immunosuppressive Effects}

Li et al. tested four C21 steroidal glycosides, namely, compounds 81, 277, 82 and 16, for their immunological activities In vitro against concanavalin A (Con A)- and lipopolysaccharide (LPS)-induced proliferation of mice splenocytes [23]. The results showed that these compounds significantly inhibit the proliferation of Con A- and LPS-induced mice splenocytes in vitro in a dose-dependent manner.

Compound 120 has a significant inhibitory effect on TNF- $\alpha$ formation on the RAW 264.7 mouse macrophage-like cell line stimulated with LPS and N9 microglial cell line stimulated with LPS/IFN- $\gamma$ (interferon- $\gamma$ ) [39].

Cho et al. investigated the anti-inflammatory effects and related molecular mechanisms of a crude polysaccharide (HMFO) which obtained from CWW in mice with dextran sulphate sodium (DSS)-induced colitis and in LPS-induced RAW 264.7 macrophages. It suggested that HMFO ameliorates the pathological characteristics of colitis and significantly reduces the production of proinflammatory cytokines in the serum [113]. Histological analysis indicated that HMFO improves the signs of histological damage. In addition, HMFO inhibits the protein expression levels of inducible nitric oxide synthase (iNOS) and cyclooxygenase-2 (COX-2) and phosphorylates the nuclear factor-kappa B (NF- $\kappa$ B) p65 levels in the colon tissue of mice with DSS-induced colitis. In macrophages, HMFO inhibits several cytokines and enzymes involved in inflammation. HMFO also attenuates inflammation both in vitro and in vivo primarily by inhibiting NF- $\kappa$ B activation. 
Zhang et al. investigated the immunosuppressive activities of compounds 335-337 and 9 isolated from $80 \%$ ethanol extract of the CA root by using an In vitro model of Con A-induced proliferation of $\mathrm{T}$ lymphocytes from mice. As a result, these four compounds exhibit strong inhibition on Con A-stimulated cell proliferation, showing $\mathrm{IC}_{50}$ values of 3.3, 7.0, 6.7 and 10.9 $\mu \mathrm{M}$ [72]. In addition, compounds 341-346, 348-350, 352-354 and 356 were assessed for their immunological activities in vitro against Con A-induced proliferation of mice splenocytes [73]. The results revealed that compounds 341, 342 and 354 at the concentration of $100 \mu \mathrm{mol} / \mathrm{L}$, compounds 343, 352 and 354 at the concentration of $10 \mu \mathrm{mol} / \mathrm{L}$ and compound 353 at the concentration of $1 \mu \mathrm{mol} / \mathrm{L}$ exhibit weak activity against the proliferation of $\mathrm{T}$ lymphocyte In vitro.

Yu et al. found that compounds 358 and 359 inhibit nitric oxide production in C57bl/6j mouse peritoneal macrophages with $17.0 \%$ and $6.9 \%$ inhibition rates, respectively, at a concentration of $10 \mu \mathrm{M}$ [74].

Fourteen steroidal glycosides were investigated by detecting the inhibitory effects of iNOS and COX-2 on RAW 246.7 murine macrophage cells stimulated by LPS [44]. The results revealed that compounds 158, 162, 156, 157, 122 and 146 can significantly inhibit iNOS expression, whereas compounds 162 and 148 can clearly inhibit COX-2 expression in RAW 246.7 cells stimulated by LPS compared with cells stimulated with LPS and not treated with other compounds.

The effects of compound 391 and extracts of CWW roots (CWE) on the expression of iNOS and proinflammatory cytokines in LPS-induced BV-2 microglial cells was investigated and the results suggested that CWE and compound 391 significantly decrease the LPS-induced NO production and the expression of iNOS in a concentration-dependent manner. Meanwhile, they did not show cytotoxic activity (CWE up to $500 \mu \mathrm{g} / \mathrm{mL}$ and compound 391 up to $80 \mu \mathrm{M}$ ). In addition, RT-PCR analysis and ELISA showed that compound 391 significantly attenuates the expression of TNF- $\alpha$, interleukin-6, and interleukin $-1 \beta$ in LPS-stimulated BV-2 cells. Furthermore, compound 391 inhibits the phosphorylation of inhibitor kappa B-alpha and translocation of NF- $\kappa$ B to the BV-2 cell nucleus. It indicates that CWE and compound 391 may exert effective anti-inflammatory activities via NF- $\kappa$ B inactivation in stimulated microglial cells [110].

Choi et al. investigated the anti-atopic dermatitis (AD) effect and molecular mechanism of the aqueous extract of CA. Topical concentrations of CA at 1 and $100 \mathrm{mg} / \mathrm{mL}$ are applied to AD-like skin lesions induced by 2,4-dinitrochlorobenzene for 11 days. Scratching behavior occurrences were evaluated for $20 \mathrm{~min}$. The results showed that topical application of CA attenuates the total serum IgE level [112].

\subsection{Anti-Oxidizing Effect}

Compound 419, a steroidal alkaloid, was isolated from CWW roots, and its effects on lipid peroxidation and the activity of aldehyde oxidase (EC. 1.2.3.1) were investigated In vitro. The results showed that it suppresses the formation of lipid peroxides in rat liver tissues significantly and potently inhibits hepatic aldehydeoxidase activity in a dose-dependent manner, with a $\mathrm{IC}_{50}$ value of $0.8 \mu \mathrm{M}$ $(0.5 \mu \mathrm{g} / \mathrm{mL})[94]$.

\subsection{Hepatoprotective Function}

Lee et al. investigated the hepatoprotective activity of compound 391 by using primary cultures of rat hepatocytes injured by $\mathrm{CCl}_{4}$. The results suggested that compound $391(50 \mu \mathrm{M})$ significantly reduces (approximately 50\%) the release into the culture medium of glutamic pyruvic transaminase and sorbitol dehydrogenase from the primary cultures of rat hepatocytes exposed to $\mathrm{CCl}_{4}$. Simultaneously, this compound ameliorates lipid peroxidation by up to $50 \%$, as demonstrated by the reduction in malondialdehyde production [114]. In addition, Jang et al. found that CWE (100 and $200 \mathrm{mg} / \mathrm{Kg}$ ) can decrease fat accumulation in the liver by suppressing COX-2, NF- $\mathrm{B}$ and p38 mitogen-activated protein kinase [115]. 


\subsection{Appetite Suppressant Effect}

Compound 96 isolated from C. auriculatum roots can suppress appetite and reduce body weight in rats. Moreover, appetite suppressant isolated from Hoodia gordonii shows significant appetite suppressing effect, resulting in weight loss in rats [30].

\subsection{Anti-Depressant Effect}

Yang et al. assessed the anti-depressant activities of compounds 294-296, 35 and 231 by using forced swimming, tail suspension and open field tests in despair mice models. The results suggested that these compounds show significant anti-depressant activities at the dosage of $50 \mathrm{mg} / \mathrm{kg}$ (i.g.). The most potential one is compound 295, with potency close to that of the positive control fluoxetine (20 mg/kg) [67].

\subsection{Vasodilating Activity}

Compound 284 was isolated from the C. stauntonii roots, and its vasodilatation activity was investigated. The results indicated that this compound exerts a dose-dependent relaxation effect on aortic rings with endothelium contracted by phenylepherine, with $\mathrm{IC}_{50}$ value of $5.37 \times 10^{-6} \mathrm{~mol} / \mathrm{L}$. The inhibitory effect of this compound on aortic rings with endothelium contracted by phenylepherine was exhibited by the relaxation effect at high concentration $\left(10^{-4} \mathrm{~mol} / \mathrm{L}\right)$, with a relaxation percentage $64.8 \% \pm 26.9 \%$. Meanwhile, compound 28 also relaxes the aorta rings contracted by $\mathrm{KCl}$ at high concentration $\left(10^{-4} \mathrm{~mol} / \mathrm{L}\right)$, with a relaxation percentage $53.4 \% \pm 7.3 \%$ [40].

Moreover, Wang et al. [116] investigated the anti-angiogenic properties of compound $\mathbf{1 7 5}$ from $C$. auriculatum. The results revealed that it can significantly inhibit the proliferation of HUVEC human umbilical vein endothelial cell proliferation and block the HUVEC migration, invasion and capillary-like tube formation by disturbing the vascular endothelial growth factor (VEGF)-VEGFR2-protein kinase B (AKT)/focal adhesion kinase signal axis.

\subsection{Others}

In addition to the pharmacological activity of the above-mentioned reviewed Cynanchum plants, compound 394 from C. bungei exerts depigmenting activity [79]. Compounds 387 and 388 from C. stauntonii exhibit anti-cardiac congestion activity [77]. Compounds 392 and 394 have an anti-platelet effect [117]. Ten-week-old female rats were ovariectomized (OVX) and treated with the aqueous extract of CWW for 1 week. The administration of CWW $(200 \mathrm{mg} / \mathrm{kg} / \mathrm{d}$ for 7 days, per os) significantly improves skin temperature increase in OVX rats [119]. Moreover, the aqueous extract of CWW inhibits the development of benign prostatic hyperplasia (BPH) in a testosterone-induced BPH rat model [120]. In addition, compound $\mathbf{2 2}$ showed an airway smooth muscle relaxant effect [12].

\section{Conclusions}

Cynanchum L. is an important genus in the Asclepiadaceae family because numerous plants in this genus show several application prospects other than in the field of medicine. Moreover, Cynanchum plants present a long history as traditional folk medicine.

At present, more than 400 compounds have been isolated from genus Cynanchum. These compounds include steroids, flavonoids, acetophenones, triterpenoids, alkaloids, phytosterols, polysaccharides and other compounds. Among these compounds, C21 steroid is the characteristic ingredient. In China, several species have been used to treat chronic diseases in TCM for thousands of years, and the roots and stems of these species have been used as a component of TCM or in combination with other Chinese medicinal plants.

Recently, increased attention has been focused on C. taiwanianum, C. auriculatum, C. paniculatum, CA, CWW, C. otophyllum and C. stauntonii because of their anti-tumor, neuroprotective, anti-fungal, 
parasitic and anti-viral, anti-depressant, anti-oxidant, anti-inflammatory and immunosuppressive effects. These plants also can suppress appetite, induce weight loss and expand blood vessels.

Although a number of reports on the chemical components and pharmacological activities of these plants are available, studies on the chemical composition are still not systematic enough because they only focus on the chemical components of several species of this genus. However, research on the pharmacological activities are mostly based on in vitro activity screening, and pharmacodynamic studies in vivo represent only a few reports. Therefore, further investigations are required for systematic research of the chemical composition and in vivo pharmacological activities of Cynanchum $\mathrm{sp}$. We believe that this work is of particular value by providing not only the fundamental insight into the medicinal value of plants in this genus; moreover, this work can provide reference for clinical medication, sustainable development and utilization of plants in this genus.

Author Contributions: C.B. reviewed the literature, discussed the layout, finished the artworks (Figures, Schemes and Tables), and finalized the paper. X.Z. reviewed the literature, discussed the layout, wrote the text and finalized the paper. L.H., W.W., L.Z., X.D. and X.Q. retrieved the relevant literature, discussed the layout, and finalized the paper. M.Y., Z.W. and S.W. retrieved the relevant literature, discussed the layout, checked for accuracy and verified that the information was factual, and finalized the paper.

Funding: This research received no external funding.

Acknowledgments: Ningxia University's First-Class Subject (Traditional Chinese Medicine) Construction Project (NXYLXK2017A06), the National Natural Science Foundation of China (No. 81460592), the Natural Science Foundation of Ningxia (No. NZ17090), the Ministry of education Chunhui project (No. Z2016064), and the Innovative education College Students Project (No. NXCX2016130) supported this work.

Conflicts of Interest: The authors declare no conflict of interest.

\section{References}

1. Wu, Z.; Ding, L.; Zhao, S. Chemical constituents and pharmacological effects of Cynanchum Linn. World Phytomed. 1991, 6, 147-154.

2. Gu, X.J.; Hao, D.C. Recent advances in phytochemistry and pharmacology of $\mathrm{C}_{21}$ steroid constituents from Cynanchum plants. Chin. J. Nat. Med. 2016, 14, 321-334. [PubMed]

3. Jiang, Y.; Choi, H.G.; Li, Y.; Park, Y.M.; Lee, J.H.; Kim, D.H.; Lee, J.H.; Son, J.K.; Na, M.; Lee, S.H. Chemical constituents of Cynanchum wilfordii and the chemotaxonomy of two species of the family asclepiadacease, C. wilfordii and C. auriculatum. Arch. Pharm. Res. 2011, 34, 2021-2027. [CrossRef] [PubMed]

4. Liu, W.; Zhang, C.; Wu, L.; Dai, Y.; Wu, Q. Research advances on chemical constituents and pharmacological actions of Cynanchum Linn. J. Chin. Med. Mater. 2003, 3, 216-218.

5. Wu, Y.; Zhou, H. Research advances on chemical constituents of Cynanchum Linn. Central South Pharm. 2006, 4, 371-375.

6. Sheng-Xiang, Q.; Zhuang-Xin, Z.; Lin, Y.; Jun, Z. Two new glycosides from the roots of Cynanchum versicolor. Planta Med. 1991, 57, 454-456. [CrossRef] [PubMed]

7. Zheng, Z.; Zhang, W.; Kong, L.; Liang, M.; Li, H.; Lin, M.; Liu, R.; Zhang, C. Rapid identification of $\mathrm{C}_{21}$ steroidal saponins in Cynanchum versicolor bunge by electrospray ionization multi-stage tandem mass spectrometry and liquid chromatography/tandem mass spectrometry. Rapid Commun. Mass Spectrom. 2007, 21, 279-285. [CrossRef] [PubMed]

8. Tang, W.; Eisenbrand, G. Cynanchum glaucescens (decne.) hand.-mazz. In Chinese Drugs of Plant Origin: Chemistry, Pharmacology, and Use in Traditional and Modern Medicine; Tang, W., Eisenbrand, G., Eds.; Springer: Berlin/Heidelberg, Germany, 1992; pp. 417-428.

9. Huang, L.-J.; Wang, B.; Zhang, J.-X.; Yan, C.; Mu, S.-Z.; Hao, X.-J. Studies on cytotoxic pregnane sapogenins from Cynanchum wilfordii. Fitoterapia 2015, 101, 107-116. [CrossRef] [PubMed]

10. Fu, Y.-W.; Zhang, Q.-Z.; Xu, D.-H.; Liang, J.-H.; Wang, B. Antiparasitic effect of cynatratoside-c from Cynanchum atratum against Ichthyophthirius multifiliis on grass carp. J. Agric. Food Chem. 2014, 62, 7183-7189. [CrossRef] [PubMed]

11. Ji-Hong, W.; Yan-Li, W.; Yu-Hua, L.; Ji-Yuan, Z.; Ze-Hong, L.I. Activity of two extracts of Cynanchum paniculatum against Ichthyophthirius multifiliis theronts and tomonts. Parasitology 2017, 144, 179-185. [CrossRef] [PubMed] 
12. Yue, G.G.; Chan, K.M.; To, M.H.; Cheng, L.; Fung, K.P.; Leung, P.C.; Lau, C.B. Potent airway smooth muscle relaxant effect of cynatratoside B, a steroidal glycoside isolated from Cynanchum stauntonii. J. Nat. Prod. 2014, 77, 1074-1077. [CrossRef] [PubMed]

13. Bai, H.; Li, W.; Koike, K. Pregnane glycosides from Cynanchum atratum. Steroids 2008, 73, 96-103. [CrossRef] [PubMed]

14. Bai, H.; Li, W.; Asada, Y.; Satou, T.; Wang, Y.; Koike, K. Twelve pregnane glycosides from Cynanchum atratum. Steroids 2009, 74, 198-207. [CrossRef] [PubMed]

15. Ma, X.-X.; Wang, D.; Zhang, Y.-J.; Yang, C.-R. Identification of new qingyangshengenin and caudatin glycosides from the roots of Cynanchum otophyllum. Steroids 2011, 76, 1003-1009. [CrossRef] [PubMed]

16. Li, J.L.; Zhou, J.; Chen, Z.H.; Guo, S.Y.; Li, C.Q.; Zhao, W.M. Bioactive $\mathrm{C}_{21}$ steroidal glycosides from the roots of Cynanchum otophyllum that suppress the seizure-like locomotor activity of zebrafish caused by pentylenetetrazole. J. Nat. Prod. 2015, 78, 1548-1555. [CrossRef] [PubMed]

17. Ma, X.-X.; Jiang, F.-T.; Yang, Q.-X.; Liu, X.-H.; Zhang, Y.-J.; Yang, C.-R. New pregnane glycosides from the roots of Cynanchum otophyllum. Steroids 2007, 72, 778-786. [CrossRef] [PubMed]

18. Shen, D.-Y.; Wei, J.-C.; Wan, J.-B.; Huang, X.-J.; Xiang, C.; Li, B.-C.; Zhang, Q.-W.; Wang, Y.-T.; Li, P. Four new $\mathrm{C}_{21}$ steroidal glycosides from Cynanchum otophyllum Schneid. Phytochem. Lett. 2014, 9, 86-91. [CrossRef]

19. Tursunova, R.N.; Maslennikova, V.A.; Abubakirov, N.K. Pregnane glycosides of Cynanchum sibiricum III. The structure of sibiricosides d and e. Chem. Nat. Compd. 1975, 11, 183-187. [CrossRef]

20. Maslennikova, V.A.; Tursunova, R.N.; Abubakirov, N.K. Pregnane glycosides of Cynanchum sibiricum. Chem. Nat. Compd. 1969, 5, 279-280. [CrossRef]

21. Gan, H.; Xiang, W.-J.; Ma, L.; Hu, L.-H. Six new $\mathrm{C}_{21}$ steroidal glycosides from Cynanchum bungei Decne. Helv. Chim. Acta 2008, 91, 2222-2234. [CrossRef]

22. Bai, H.; Li, W.; Koike, K.; Satou, T.; Chen, Y.; Nikaido, T. Cynanosides a-j, ten novel pregnane glycosides from Cynanchum atratum. Tetrahedron 2005, 61, 5797-5811. [CrossRef]

23. Li, X.; Sun, H.; Ye, Y.; Chen, F.; Pan, Y. C-21 steroidal glycosides from the roots of Cynanchum chekiangense and their immunosuppressive activities. Steroids 2006, 71, 61-66. [CrossRef] [PubMed]

24. Tai, Y.; Cao, X.; Li, X.; Pan, Y. Identification of C-21 steroidal glycosides from the roots of Cynanchum chekiangense by high-performance liquid chromatography/tandem mass spectrometry. Anal. Chim. Acta 2006, 572, 230-236. [CrossRef] [PubMed]

25. Lee, K.Y.; Sung, S.H.; Kim, Y.C. New acetylcholinesterase-inhibitory pregnane glycosides of Cynanchum atratum roots. Helv. Chim. Acta 2003, 86, 474-483. [CrossRef]

26. Xiang, W.-J.; Ma, L.; Hu, L.-H. $C_{21}$ steroidal glycosides from Cynanchum wilfordii. Helv. Chim. Acta 2009, 92, 2659-2674. [CrossRef]

27. Yoon, M.-Y.; Choi, N.H.; Min, B.S.; Choi, G.J.; Choi, Y.H.; Jang, K.S.; Han, S.-S.; Cha, B.; Kim, J.-C. Potent in vivo antifungal activity against powdery mildews of pregnane glycosides from the roots of Cynanchum wilfordii. J. Agric. Food Chem. 2011, 59, 12210-12216. [CrossRef] [PubMed]

28. Lin, Y.-L.; Lin, T.-C.; Kuo, Y.-H. Five new pregnane glycosides from Cynanchum taiwanianum. J. Nat. Prod. 1995, 58, 1167-1173. [CrossRef] [PubMed]

29. Gu, X.-J.; Yao, N.; Qian, S.-H.; Li, Y.-B.; Li, P. Four new $\mathrm{C}_{21}$ steroidal glycosides from the roots of Cynanchum auriculatum. Helv. Chim. Acta 2009, 92, 88-97. [CrossRef]

30. Liu, S.; Chen, Z.; Wu, J.; Wang, L.; Wang, H.; Zhao, W. Appetite suppressing pregnane glycosides from the roots of Cynanchum auriculatum. Phytochemistry 2013, 93, 144-153. [CrossRef] [PubMed]

31. Li, X.; Luo, Y.; Li, G.P.; Yang, Q.X. Pregnane glycosides from the antidepressant active fraction of cultivated Cynanchum otophyllum. Fitoterapia 2016, 110, 96-102. [CrossRef] [PubMed]

32. Chen, H.; Xu, N.; Zhou, Y.; Qiao, L.; Cao, J.; Yao, Y.; Hua, H.; Pei, Y. Steroidal glycosides from the roots of Cynanchum amplexicaule Sieb. et Zucc. Steroids 2008, 73, 629-636. [CrossRef] [PubMed]

33. Liqin, W.; Yuemao, S.; Xing, X.; Yuqing, W.; Jun, Z. Five new $\mathrm{C}_{21}$ steroidal glycosides from Cynanchum komarovii al.Iljinski. Steroids 2004, 69, 319-324. [CrossRef] [PubMed]

34. Konda, Y.; Toda, Y.; Harigaya, Y.; Lou, H.; Li, X.; Onda, M. Two new glycosides, hancoside and neohancoside a, from Cynanchum hancockianum. J. Nat. Prod. 1992, 55, 1447-1453. [CrossRef]

35. Dou, J.; Li, P.; Bi, Z.M.; Zhou, J.L. New c21 steroidal glycoside from Cynanchum paniculatum. Chin. Chem. Lett. 2007, 18, 300-302. [CrossRef] 
36. Yan, Y.; Zhang, J.X.; Liu, K.X.; Huang, T.; Yan, C.; Huang, L.J.; Liu, S.; Mu, S.Z.; Hao, X.J. Seco-pregnane steroidal glycosides from the roots of Cynanchum atratum and their anti-TMV activity. Fitoterapia 2014, 97, 50-63. [CrossRef] [PubMed]

37. Konda, Y.; Toda, Y.; Takayanagi, H.; Ogura, H.; Harigaya, Y.; Lou, H.; Li, X.; Onda, M. A new modified steroid, hancopregnane, and a new monoterpene from Cynanchum hancockianum. J. Nat. Prod. 1992, 55, 1118-1123. [CrossRef]

38. Fu, M.H.; Wang, Z.J.; Yang, H.J.; Maimai, M.; Fang, J.; Tang, L.Y.; Yang, L. A new $\mathrm{C}_{21}$-steroidal glycoside from Cynanchum stauntonii. Chin. Chem. Lett. 2007, 18, 415-417. [CrossRef]

39. Day, S.-H.; Wang, J.-P.; Won, S.-J.; Lin, C.-N. Bioactive constituents of the roots of Cynanchum atratum. J. Nat. Prod. 2001, 64, 608-611. [CrossRef] [PubMed]

40. Wang, P.; Qin, H.-L.; Zhang, L.; Li, Z.-H.; Wang, Y.-H.; Zhu, H.-B. Steroids from the roots of Cynanchum stauntonii. Planta Med. 2004, 70, 1075-1079. [CrossRef] [PubMed]

41. Dou, J.; Li, P.; Song, Y.; Qi, L.W.; Bi, Z.M. Application of liquid chromatography coupled with electrospray ionization time-of-flight mass spectrometry for screening and quantitative analysis of $\mathrm{C}_{21}$ steroids in the roots and rhizomes of Cynanchum paniculatum. J. Sep. Sci. 2007, 30, 992-998. [CrossRef] [PubMed]

42. Zhu, N.; Wang, M.; Kikuzaki, H.; Nakatani, N.; Ho, C.-T. Two $\mathrm{C}_{21}$-steroidal glycosides isolated from Cynanchum stauntoi. Phytochemistry 1999, 52, 1351-1355. [CrossRef]

43. Yu, J.-Q.; Deng, A.-J.; Qin, H.-L. Nine new steroidal glycosides from the roots of Cynanchum stauntonii. Steroids 2013, 78, 79-90. [CrossRef] [PubMed]

44. Lai, C.-Z.; Liu, J.-X.; Pang, S.-W.; Dai, Y.; Zhou, H.; Mu, Z.-Q.; Wu, J.; Tang, J.-S.; Liu, L.; Yao, X.-S. Steroidal glycosides from the roots of Cynanchum stauntonii and their effects on the expression of iNOS and COX-2. Phytochem. Lett. 2016, 16, 38-46. [CrossRef]

45. Deng, A.J.; Yu, J.Q.; Li, Z.H.; Ma, L.; Zhang, Z.H.; Qin, H.L. 14,15-secopregnane-type glycosides with 5alpha:9alpha-peroxy and delta(6,8(14))-diene linkages from the roots of Cynanchum stauntonii. Molecules 2017, 22, 860. [CrossRef] [PubMed]

46. Zhang, M.; Li, X.; Xiang, C.; Qin, Y.; He, J.; Li, B.C.; Li, P. Cytotoxicity of pregnane glycosides of Cynanchum otophyllum. Steroids 2015, 104, 49-60. [CrossRef] [PubMed]

47. Ma, L.-F.; Shan, W.-G.; Zhan, Z.-J. Polyhydroxypregnane glycosides from the roots of Cynanchum otophyllum. Helv. Chim. Acta 2011, 94, 2272-2282. [CrossRef]

48. Peng, Y.R.; Li, Y.B.; Liu, X.D.; Zhang, J.F.; Duan, J.A. Antitumor activity of C-21 steroidal glycosides from Cynanchum auriculatum Royle ex Wight. Phytomedicine 2008, 15, 1016-1020. [CrossRef] [PubMed]

49. Zhao, Y.-B.; He, H.-P.; Lu, C.-H.; Mu, Q.-Z.; Shen, Y.-M.; Hao, X.-J. C 21 steroidal glycosides of seven sugar residues from Cynanchum otophyllum. Steroids 2006, 71, 935-941. [CrossRef] [PubMed]

50. Li, J.L.; Gao, Z.B.; Zhao, W.M. Identification and evaluation of antiepileptic activity of $C_{21}$ steroidal glycosides from the roots of Cynanchum wilfordii. J. Nat. Prod. 2016, 79, 89-97. [CrossRef] [PubMed]

51. Zhao, Y.; Shen, Y.; He, H.; Du, Z.; Mu, Q.; Hao, X. $\mathrm{C}_{21}$ steroidal saponins from Cynanchum otophyllum. Chin. Herb. Med. 2015, 7, 273-278. [CrossRef]

52. Yang, X.-X.; Bao, Y.-R.; Wang, S.; Zhu, R.-Q.; Bao, L.-N.; Guan, Y.-P.; Meng, X.-S. Steroidal glycosides from roots of Cynanchum otophyllum. Chem. Nat. Compd. 2015, 51, 703-705. [CrossRef]

53. Wang, D.; Bao, Y.-R. A new steroidal glycoside from roots of Cynanchum wallichii. Chem. Nat. Compd. 2015, 51, 897-899. [CrossRef]

54. Hwang, B.Y.; Kim, S.E.; Kim, Y.H.; Kim, H.S.; Hong, Y.-S.; Ro, J.S.; Lee, K.S.; Lee, J.J. Pregnane glycoside multidrug-resistance modulators from Cynanchum wilfordii. J. Nat. Prod. 1999, 62, 640-643. [CrossRef] [PubMed]

55. Zhang, M.; Rao, L.L.; Xiang, C.; Li, B.C.; Li, P. C 21 steroidal glycosides from the roots of Cynanchum saccatum. Steroids 2015, 101, 28-36. [CrossRef] [PubMed]

56. Rao, L.-L.; Zhang, M.; Xiang, C.; Li, B.-C.; Li, P. Steroid glycosides and phenols from the roots of Cynanchum saccatum. Phytochem. Lett. 2015, 11, 49-52. [CrossRef]

57. Qi, L.W.; Gu, X.J.; Li, P.; Liang, Y.; Hao, H.; Wang, G. Structural characterization of pregnane glycosides from Cynanchum auriculatum by liquid chromatography on a hybrid ion trap time-of-flight mass spectrometer. Rapid Commun. Mass Spectrom. 2009, 23, 2151-2160. [CrossRef] [PubMed]

58. Kim, C.S.; Oh, J.Y.; Choi, S.U.; Lee, K.R. Chemical constituents from the roots of Cynanchum paniculatum and their cytotoxic activity. Carbohydr. Res. 2013, 381, 1-5. [CrossRef] [PubMed] 
59. Liu, Y.; Hu, Y.; Yu, S.; Fu, G.; Huang, X.; Fan, L. Steroidal glycosides from Cynanchum forrestii Schlechter. Steroids 2006, 71, 67-76. [CrossRef] [PubMed]

60. Liu, Y.; Qu, J.; Yu, S.-S.; Hu, Y.-C.; Huang, X.-Z. Seven new steroidal glycosides from the roots of Cynanchum forrestii. Steroids 2007, 72, 313-322. [CrossRef] [PubMed]

61. Li, S.L.; Tan, H.; Shen, Y.M.; Kawazoe, K.; Hao, X.J. A pair of new C-21 steroidal glycoside epimers from the roots of Cynanchum paniculatum. J. Nat. Prod. 2004, 67, 82-84. [CrossRef] [PubMed]

62. Lou, H.; Li, X.; Onda, M.; Konda, Y.; Machida, T.; Toda, Y.; Harigaya, Y. Further isolation of glycosides from Cynanchum hancockianum. J. Nat. Prod. 1993, 56, 1437-1443. [CrossRef] [PubMed]

63. Yu, J.-Q.; Zhao, L. Two new glycosides and one new neolignan from the roots of Cynanchum stauntonii. Phytochem. Lett. 2015, 13, 355-359. [CrossRef]

64. Deng, A.J.; Zhang, D.; Li, Q.; Zhang, Z.H.; Li, Z.H.; Qin, H.L. Sugar-free pregnane-type steroids from the roots of Cynanchum stauntonii. J. Asian Nat. Prod. Res. 2017, 19, 557-563. [CrossRef] [PubMed]

65. Chen, G.; Chen, H.; Li, W.; Pei, Y.H. Steroidal glycosides from Cynanchum amplexicaule. J. Asian Nat. Prod. Res. 2011, 13, 756-760. [CrossRef] [PubMed]

66. Yeo, H.; Kim, K.W.; Kim, J.; Choi, Y.H. Steroidal glycosides of the 14,15-seco-18-nor-pregnane series from Cynanchum ascyrifolium. Phytochemistry 1998, 49, 1129-1133. [CrossRef]

67. Yang, Q.-X.; Ge, Y.-C.; Huang, X.-Y.; Sun, Q.-Y. Cynanauriculoside C-E, three new antidepressant pregnane glycosides from Cynanchum auriculatum. Phytochem. Lett. 2011, 4, 170-175. [CrossRef]

68. Huang, X.; Tan, A.-M.; Yang, S.-B.; Zhang, A.-Y.; Zhang, H. Two new C21 steroidal glycosides from the stems of Cynanchum paniculatumkitag. Helv. Chim. Acta 2009, 92, 937-943. [CrossRef]

69. Lu, Y.; Teng, H.-L.; Yang, G.-Z.; Mei, Z.-N. Three new steroidal glycosides from the roots of Cynanchum auriculatum. Helv. Chim. Acta 2011, 94, 1296-1303. [CrossRef]

70. Zhao, Y.; Fan, Q.; Xu, G.; Feng, Z.; Hao, X. $C_{21}$ steroidal glycosides from acidic hydrolysate of Cynanchum otophyllum. Chin. Herb. Med. 2014, 6, 319-323. [CrossRef]

71. Zhao, Z.-M.; Sun, Z.-H.; Chen, M.-H.; Liao, Q.; Tan, M.; Zhang, X.-W.; Zhu, H.-D.; Pi, R.-B.; Yin, S. Neuroprotective polyhydroxypregnane glycosides from Cynanchum otophyllum. Steroids 2013, 78, 1015-1020. [CrossRef] [PubMed]

72. Zhang, Z.J.; Ding, M.L.; Tao, L.J.; Zhang, M.; Xu, X.H.; Zhang, C.F. Immunosuppressive $\mathrm{C}_{21}$ steroidal glycosides from the root of Cynanchum atratum. Fitoterapia 2015, 105, 194-201. [CrossRef] [PubMed]

73. Cui, B.; Wang, X.; Yang, Y.; Yang, Y.; Shi, S.; Guo, F.; Li, Y. Sixteen novel C-21 steroidal glycosides from the roots of Cynanchum mooreanum. Steroids 2015, 104, 79-94. [CrossRef] [PubMed]

74. Yu, J.-Q.; Zhao, L. Seco-pregnane steroidal glycosides from the roots of Cynanchum stauntonii. Phytochem. Lett. 2016, 16, 34-37. [CrossRef]

75. Tsoukalas, M.; Psichas, A.; Reimann, F.; Gribble, F.M.; Lobstein, A.; Urbain, A. Pregnane glycosides from Cynanchum menarandrense. Steroids 2017, 125, 27-32. [CrossRef] [PubMed]

76. Sheng, F.; Chen, M.; Tan, Y.; Xiang, C.; Zhang, M.; Li, B.; Su, H.; He, C.; Wan, J.; Li, P. Protective effects of otophylloside $\mathrm{n}$ on pentylenetetrazol-induced neuronal injury in vitro and in vivo. Front. Pharmacol. 2016, 7, 224. [CrossRef] [PubMed]

77. Shibano, M.; Misaka, A.; Sugiyama, K.; Taniguchi, M.; Baba, K. Two secopregnane-type steroidal glycosides from Cynanchum stauntonii (Decne.) Schltr.ex Levl. Phytochem. Lett. 2012, 5, 304-308. [CrossRef]

78. Huang, P.-L.; Won, S.-J.; Day, S.-H.; Lin, C.-N. A cytotoxic acetophenone with a novel skeleton, isolated from Cynanchum taiwanianum. Helv. Chim. Acta 1999, 82, 1716-1720. [CrossRef]

79. Ding, H.-Y.; Chang, T.-S.; Shen, H.-C.; Tai, S.S.-K. Murine tyrosinase inhibitors from Cynanchum bungei and evaluation of in vitro and in vivo depigmenting activity. Exp. Dermatol. 2011, 20, 720-724. [CrossRef] [PubMed]

80. Weon, J.B.; Lee, B.; Yun, B.R.; Lee, J.; Ma, C.J. Simultaneous determination of ten bioactive compaounds from the roots of Cynanchum paniculatum by using high performance liquid chromatography coupled-diode array detector. Pharmacogn. Mag. 2012, 8, 231-236. [PubMed]

81. Weon, J.B.; Kim, C.Y.; Yang, H.J.; Ma, C.J. Neuroprotective compounds isolated from Cynanchum paniculatum. Arch. Pharm. Res. 2012, 35, 617-621. [CrossRef] [PubMed]

82. Lin, Y.-L.; Lin, T.-C.; Kuo, Y.-H. Two acetophenone glucosides, cynanonesides A and B, from Cynanchum taiwanianum and revision of the structure for cynandione a. J. Nat. Prod. 1997, 60, 368-370. [CrossRef] 
83. Sun, Y.; Liu, Z.; Wang, J.; Xiang, L.; Zhu, L. Separation and purification of baishouwubenzophenone, 4-hydroxyacetophenone and 2,4-dihydroxyacetophenone from Cynanchum auriculatum Royle ex Wight by HSCCC. Chromatographia 2009, 70, 1-6. [CrossRef]

84. Yeo, H.; Kim, J. A benzoquinone from Cynanchum wilfordii. Phytochemistry 1997, 46, 1103-1105. [CrossRef]

85. Chen, Z.-S.; Lai, J.-S.; Kao, Y.-H. The constituents of Cynanchum taiwanianum. J. Chin. Chem. Soc. 1991, 38, 393-396. [CrossRef]

86. Niu, Y.-L.; Chen, X.; Wu, Y.; Jiang, H.-Q.; Zhang, X.-L.; Li, E.-T.; Li, Y.-Y.; Zhou, H.-L.; Liu, J.-G.; Wang, D.-Y. Chemical constituents from Cynanchum paniculatum (Bunge) Kitag. Biochem. Syst. Ecol. 2015, 61, 139-142. [CrossRef]

87. Lee, D.-U.; Kang, S.-I.; Yoon, S.-H.; Budesinsky, M.; Kasal, A.; Mayer, K.K.; Wiegrebe, W. A new steroidal alkaloid from the roots of Cynanchum caudatum. Planta Med. 2000, 66, 480-482. [CrossRef] [PubMed]

88. Tanner, U.; Wiegrebe, W. Alkaloids of Cynanchum vincetoxicum: Efficacy against MDA-MB-231 mammary carcinoma cells. Arch. Pharm. (Weinheim) 1993, 326, 67-72. [CrossRef] [PubMed]

89. Budzikiewicz, H.; Faber, L.; Herrmann, E.-G.; Perrollaz, F.F.; Schlunegger, U.P.; Wiegrebe, W. Vinceten, ein benzopyrroloisochinolin-alkaloid, aus Cynanchum vincetoxicum (L.) pers. (Asclepiadaceae). Liebigs Annalen der Chemie 1979, 1979, 1212-1231. [CrossRef]

90. Stærk, D.; Christensen, J.; Lemmich, E.; Duus, J.Ø.; Olsen, C.E.; Jaroszewski, J.W. Cytotoxic activity of some phenanthroindolizidine $\mathrm{N}$-oxide alkaloids from Cynanchum vincetoxicum. J. Nat. Prod. 2000, 63, 1584-1586. [CrossRef] [PubMed]

91. Staerk, D.; Lykkeberg, A.K.; Christensen, J.; Budnik, B.A.; Abe, F.; Jaroszewski, J.W. In vitro cytotoxic activity of phenanthroindolizidine alkaloids from Cynanchum vincetoxicum and tylophora tanakae against drug-sensitive and multidrug-resistant cancer cells. J. Nat. Prod. 2002, 65, 1299-1302. [PubMed]

92. An, T.; Huang, R.-Q.; Yang, Z.; Zhang, D.-K.; Li, G.-R.; Yao, Y.-C.; Gao, J. Alkaloids from cynanchum komarovii with inhibitory activity against the tobacco mosaic virus. Phytochemistry 2001, 58, 1267-1269. [CrossRef]

93. Liu, H.; Gao, Y.; Wang, K.; Hu, Z. Determination of active components in Cynanchum chinense R. Br. by capillary electrophoresis. Biomed. Chromatogr. 2006, 20, 451-454. [CrossRef] [PubMed]

94. Lee, D.-U.; Shin, U.-S.; Huh, K. Inhibitory effects of gagaminine, a steroidal alkaloid from Cynanchum wilfordi, on lipid peroxidation and aldehyde oxidase activity. Planta Med. 1996, 62, 485-487. [CrossRef] [PubMed]

95. Konda, Y.; Toida, T.; Kaji, E.; Takeda, K.; Harigaya, Y. First total synthesis of two new diglycosides, neohancosides a and b, from Cynanchum hancockianum. Carbohydrate Res. 1997, 301, 123-143. [CrossRef]

96. Shan, L.; Zhang, W.D.; Zhang, C.; Liu, R.H.; Su, J.; Zhou, Y. Antitumor activity of crude extract and fractions from root tuber of Cynanchum auriculatum Royle ex Wight. Phytother. Res. 2005, 19, 259-261. [CrossRef] [PubMed]

97. Hu, S.; Zhao, J.; Wang, S.; Han, J. The mechanism of antitumor activity of total glucosides extracted from Cynanchum auriculatum royle (CA). Chin. J. Cancer Res. 1989, 1, 33-40. [CrossRef]

98. Li, Y.; Zhang, J.; Gu, X.; Peng, Y.; Huang, W.; Qian, S. Two new cytotoxic pregnane glycosides from Cynanchum auriculatum. Planta Med. 2008, 74, 551-554. [CrossRef] [PubMed]

99. Zhang, R.-S.; Ye, Y.-P.; Shen, Y.-M.; Liang, H.-L. Two new cytotoxic C-21 steroidal glycosides from the root of Cynanchum auriculatum. Tetrahedron 2000, 56, 3875-3879. [CrossRef]

100. Lee, S.K.; Nam, K.A.; Heo, Y.H. Cytotoxic activity and G2/M cell cycle arrest mediated by antofine, a phenanthroindolizidine alkaloid isolated from Cynanchum paniculatum. Planta Med. 2003, 69, 21-25. [CrossRef] [PubMed]

101. Yin, Z.Q.; Yu, S.L.; Wei, Y.J.; Ma, L.; Wu, Z.F.; Wang, L.; Zhang, Q.W.; Zhao, M.; Ye, W.C.; Che, C.T.; et al. C 21 steroidal glycosides from Cynanchum stauntonii induce apoptosis in HepG2 cells. Steroids 2016, 106, 55-61. [CrossRef] [PubMed]

102. Zhao, D.; Feng, B.; Chen, S.; Chen, G.; Li, Z.; Lu, X.; Sang, X.; An, X.; Wang, H.; Pei, Y. C 21 steroidal glycosides from the roots of Cynanchum paniculatum. Fitoterapia 2016, 113, 51-57. [CrossRef] [PubMed]

103. Zhang, J.; Ma, L.; Wu, Z.F.; Yu, S.L.; Wang, L.; Ye, W.C.; Zhang, Q.W.; Yin, Z.Q. Cytotoxic and apoptosis-inducing activity of $C_{21}$ steroids from the roots of Cynanchum atratum. Steroids 2017, 122, 1-8. [CrossRef] [PubMed] 
104. Lee, M.K.; Yeo, H.; Kim, J.; Markelonis, G.J.; Oh, T.H.; Kim, Y.C. Cynandione a from Cynanchum wilfordii protects cultured cortical neurons from toxicity induced by $\mathrm{H}_{2} \mathrm{O}_{2}$, L-glutamate, and kainate. J. Neurosci. Res. 2000, 59, 259-264. [CrossRef]

105. Lee, K.Y.; Yoon, J.S.; Kim, E.S.; Kang, S.Y.; Kim, Y.C. Anti-acetylcholinesterase and anti-amnesic activities of a pregnane glycoside, cynatroside b, from Cynanchum atratum. Planta Med. 2005, 71, 7-11. [CrossRef] [PubMed]

106. Yang, J.; Huang, X.B.; Wan, Q.L.; Ding, A.J.; Yang, Z.L.; Qiu, M.H.; Sun, H.Y.; Qi, S.H.; Luo, H.R. Otophylloside $\mathrm{b}$ protects against abeta toxicity in Caenorhabditis elegans models of alzheimer's disease. Nat. Prod. Bioprospect. 2017, 7, 207-214. [CrossRef] [PubMed]

107. Kim, W.; Oh, T.S.; Park, Y.J. Anti-viral effect of herbal medicine korean traditional Cynanchum paniculatum (BGE.) kitag extracts. Afr J. Tradit. Complement Altern. Med. 2017, 14, 194-198. [CrossRef] [PubMed]

108. Kim, M.-G.; Yang, J.-Y.; Lee, H.-S. Acaricidal potentials of active properties isolated from Cynanchum paniculatum and acaricidal changes by introducing functional radicals. J. Agric. Food Chem. 2013, 61, 7568-7573. [CrossRef] [PubMed]

109. Yang, Z.-C.; Wang, B.-C.; Yang, X.-S.; Wang, Q. Chemical composition of the volatile oil from Cynanchum stauntonii and its activities of anti-influenza virus. Colloids Surf. B Biointerfaces 2005, 43, 198-202.

110. Yang, S.B.; Lee, S.M.; Park, J.H.; Lee, T.H.; Baek, N.I.; Park, H.J.; Lee, H.; Kim, J. Cynandione a from Cynanchum wilfordii attenuates the production of inflammatory mediators in LPS-induced BV-2 microglial cells via NF-kB inactivation. Biol. Pharm. Bull. 2014, 37, 1390-1396. [CrossRef] [PubMed]

111. Yu, J.Q.; Lin, M.B.; Deng, A.J.; Hou, Q.; Bai, J.Y.; Li, Z.H.; Ma, L.; Zhang, Z.H.; Yuan, S.P.; Jiang, R.T.; et al. 14,15-secopregnane-type $\mathrm{C}_{21}$-steriosides from the roots of Cynanchum stauntonii. Phytochemistry 2017, 138, 152-162. [CrossRef] [PubMed]

112. Choi, Y.Y.; Kim, M.H.; Lee, H.; Ahn, K.S.; Um, J.Y.; Lee, S.G.; Kim, J.; Yang, W.M. Cynanchum atratum inhibits the development of atopic dermatitis in 2,4-dinitrochlorobenzene-induced mice. Biomed. Pharmacother. 2017, 90, 321-327. [CrossRef] [PubMed]

113. Cho, C.W.; Ahn, S.; Lim, T.G.; Hong, H.D.; Rhee, Y.K.; Yang, D.C.; Jang, M. Cynanchum wilfordii polysaccharides suppress dextran sulfate sodium-induced acute colitis in mice and the production of inflammatory mediators from macrophages. Mediators Inflamm. 2017, 2017, 3859856. [CrossRef] [PubMed]

114. Lee, M.K.; Yeo, H.; Kim, J.; Kim, Y.C. Protection of rat hepatocytes exposed to $\mathrm{CCl}_{4}$ in-vitro by cynandione a, a biacetophenone from Cynanchum wilfordii. J. Pharm. Pharmacol. 2000, 52, 341-345. [CrossRef] [PubMed]

115. Jang, S.A.; Lee, S.; Sohn, E.H.; Yang, J.; Park, D.W.; Jeong, Y.J.; Kim, I.; Kwon, J.E.; Song, H.S.; Cho, Y.M.; et al. Cynanchum wilfordii radix attenuates liver fat accumulation and damage by suppressing hepatic cyclooxygenase- 2 and mitogen-activated protein kinase in mice fed with a high-fat and high-fructose diet. Nutr. Res. 2016, 36, 914-924. [CrossRef] [PubMed]

116. Wang, X.; Fu, X.; Zhao, S.; Fu, X.; Zhang, H.; Shao, L.; Li, G.; Fan, C. Antiangiogenic properties of caudatin in vitro and in vivo by suppression of VEGF-VEGFR2-AKT/FAK signal axis. Mol. Med. Rep. 2017, 16, 8937-8943. [CrossRef] [PubMed]

117. Lin, C.N.; Huang, P.L.; Wang, J.J.; Day, S.H.; Lin, H.C.; Wang, J.P.; Ko, Y.L.; Teng, C.M. Stereochemistry and biological activities of constituents from Cynanchum taiwanianum. Biochim. Biophys. Acta 1998, 1380, 115-122. [CrossRef]

118. Devitt, G.; Howard, K.; Mudher, A.; Mahajan, S. Raman spectroscopy: An emerging tool in neurodegenerative disease research and diagnosis. ACS Chem. Neurosci. 2018, 9, 404-420. [CrossRef] [PubMed]

119. Lee, G.; Choi, C.Y.; Jun, W. Effects of aqueous extracts of Cynanchum wilfordii in rat models for postmenopausal hot flush. Prev. Nutr. Food Sci. 2016, 21, 373-377. [CrossRef] [PubMed]

120. Lee, G.; Shin, J.; Choi, H.; Jo, A.; Pan, S.; Bae, D.; Lee, Y.; Choi, C. Cynanchum wilfordii ameliorates testosterone-induced benign prostatic hyperplasia by regulating 5alpha-reductase and androgen receptor activities in a rat model. Nutrients 2017, 9, 1070. [CrossRef] [PubMed]

(c) 2018 by the authors. Licensee MDPI, Basel, Switzerland. This article is an open access article distributed under the terms and conditions of the Creative Commons Attribution (CC BY) license (http:/ / creativecommons.org/licenses/by/4.0/). 